Florida International University

FIU Digital Commons

6-28-2019

\title{
Assessing Cognitive Interview Mnemonics and Their Effectiveness with Non-Native English Speakers
}

Bryan Keith Wylie

Florida International University, bwyli002@fiu.edu

Follow this and additional works at: https://digitalcommons.fiu.edu/etd

Part of the Law and Psychology Commons, and the Psychology Commons

\section{Recommended Citation}

Wylie, Bryan Keith, "Assessing Cognitive Interview Mnemonics and Their Effectiveness with Non-Native English Speakers" (2019). FIU Electronic Theses and Dissertations. 4239.

https://digitalcommons.fiu.edu/etd/4239

This work is brought to you for free and open access by the University Graduate School at FIU Digital Commons. It has been accepted for inclusion in FIU Electronic Theses and Dissertations by an authorized administrator of FIU Digital Commons. For more information, please contact dcc@fiu.edu. 


\section{FLORIDA INTERNATIONAL UNIVERSITY}

Miami, Florida

\section{ASSESSING COGNITIVE INTERVIEW MNEMONICS AND THEIR EFFECTIVENESS WITH NON-NATIVE ENGLISH SPEAKERS}

A dissertation submitted in partial fulfillment of

the requirements for the degree of

DOCTOR OF PHILOSOPHY

in

PSYCHOLOGY

by

Bryan Keith Wylie

2019 
To: Dean Michael R. Heithaus

College of Arts, Sciences and Education

This dissertation, written by Bryan Keith Wylie, and entitled Assessing Cognitive Interview Mnemonics and Their Effectiveness with Non-Native English Speakers, having been approved in respect to style and intellectual content, is referred to you for judgment.

We have read this dissertation and recommend that it be approved.

$\begin{array}{r}\hline \text { Melissa Baralt } \\ \hline \text { Ronald Fisher } \\ \hline \text { Deborah Goldfarb } \\ \hline \text { Jacqueline Evans, Major Professor }\end{array}$

Date of Defense: June 28, 2019

The dissertation of Bryan Keith Wylie is approved.

Dean Michael R. Heithaus

College of Arts, Sciences and Education

Andrés G. Gil

Vice President for Research and Economic Development and Dean of the University Graduate School

Florida International University, 2019 


\section{DEDICATION}

This dissertation is dedicated to my parents. Thank you for believing in me, supporting me, and being excited about my work.

It is also dedicated to Taylor, who would be furious if she weren't mentioned somewhere. Thank you for putting up with me for almost 20 years. Here's to at least a few more. 


\section{ACKNOWLEDGMENTS}

"If I have seen further it is by standing on the shoulders of giants."

— Isaac Newton, Letter to Robert Hook, 1675

First and foremost, I want to thank my committee and professors. Dr. Steve Charman, your mentorship from my first day at FIU has been instrumental in my development as a researcher and as a teacher. Dr. Nadja Schreiber Compo, your support for the project and expertise in vulnerable witness interviewing has been invaluable. Dr. Melissa Baralt, your enthusiasm for the project and your guidance with the psycholinguistic background has made this a stronger dissertation. Dr. Ron Fisher, your feedback throughout my journey in the program has improved my writing quality and critical thinking; I will forever revise my work by asking myself, "What would Ron say?" Dr. Debbie Goldfarb, your excitement and support throughout my dissertation and job search has kept me from losing it on more than one occasion; thank you. And above all, Dr. Jacki Evans: thank you for your guidance_-in research, writing, teaching, and problem solving — over the past five years. Thank you for believing in me, especially when I wasn't sure I believed in myself.

Thanks to Taylor and Sarai for their friendship during grad school and for many years before. I am incredibly lucky to have my legal buddy, Allison; my third-floor office companions, Amelia and Michelle; and my friends and colleagues who have thrived through grad school with me: Alexis, Ali, Andrea, Brian, Chris, Danielle, Kelsey, Kureva, Laura, Olivia, Sarah, Stefanie, and Tiffany.

This dissertation would have never gotten done without the help of an incredible team of undergrad researchers. Thank you to Alex Perry, Bianca Vasilik, Jonathan 
Morera, Katia Suris, Laura Matzdorf, Luis Lovo, Nicolas Nieto, Robert Vazquez, and Ruquyyah Smith. Your tireless efforts have made running the study and analyzing the data possible.

Thank you to Mr. Luis Sanchez and the staff at FIU's English Language Institute for permitting and assisting with recruitment from their classes.

I would like to acknowledge the American Psychology-Law Society Grant-in-Aid and the FIU Psychology Department Seed Funds for supporting this project financially; their support helped us recruit participants from the English Language Institute and from the Miami community.

And most importantly, thank you to my family for being by my side during this journey. 


\section{ABSTRACT OF THE DISSERTATION \\ ASSESSING COGNITIVE INTERVIEW MNEMONICS AND THEIR EFFECTIVENESS WITH NON-NATIVE ENGLISH SPEAKERS}

by

\section{Bryan Keith Wylie}

Florida International University, 2019

Miami, Florida

\section{Professor Jacqueline Evans, Major Professor}

The cognitive interview is a widely recommended forensic interviewing strategy which elicits more details than comparison interviews. However, little research has attended to which of its component mnemonics drive the overall effect. Furthermore, some mnemonics - like asking witnesses to recall in reverse order — are cognitively demanding. Responding to cognitively demanding interview mnemonics may be challenging for witnesses who are already under heavy cognitive load, such as non-native English speakers. Speaking a second language is a cognitively difficult task that may leave nonnative English speakers with limited cognitive resources to devote to complex interviewing mnemonics. Other mnemonics, though, may be particularly beneficial for non-native English speakers. For example, a transfer of control instruction, emphasizing that the witness has critical knowledge the interviewer needs to know, may help nonnative English speakers overcome social barriers to reporting details in forensic interviews. The present study tests the effectiveness of the reverse order mnemonic and the transfer of control instruction compared to control interviews among native and nonnative English speakers. Native speakers $(N=64)$ and non-native English speakers $(N=$ 
34) watched a mock crime video, completed a language history questionnaire, and were interviewed about the crime video using either a control (free recall + second recall attempt), reverse order (free recall + reverse order recall attempt), or transfer of control (instruction + free recall) protocol. Native English speakers provided more correct units than non-native English speakers, especially in the control condition's second recall attempt (compared to the reverse order recall attempt). The transfer of control instruction had no effect on number of correct units provided in the first recall attempt of each condition. Accuracy rates were unaffected by language or interview condition, but nonnative English speakers, particularly in the transfer of control condition, provided somewhat higher proportions of subjective details than native English speakers. These results suggest that non-native English speakers provide fewer details than native English speakers when interviewed in English, and the two mnemonics tested have little influence on speakers' output. Future research should develop an interviewing protocol that is sensitive to the challenges faced by non-native speakers. 


\section{TABLE OF CONTENTS}

CHAPTER

PAGE

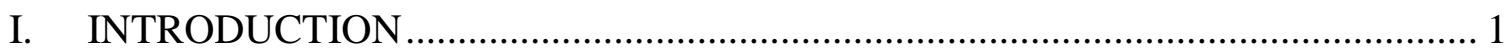

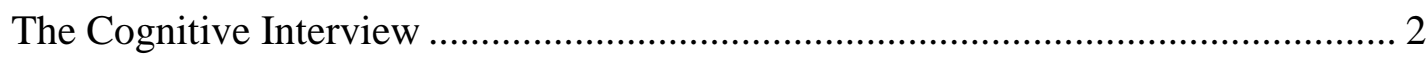

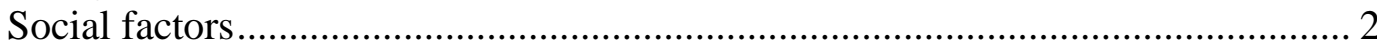

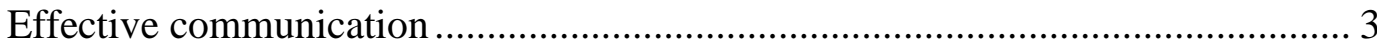

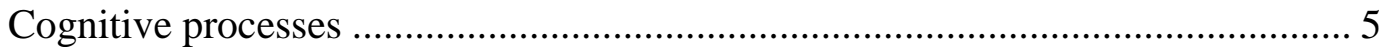

Empirical support for the CI .............................................................................

Language, Cognition, and Memory …………………….................................... 12

Investigative Interviewing of Non-Native Speakers................................................ 19

Linguistic Properties of Complex Tasks ................................................................ 22

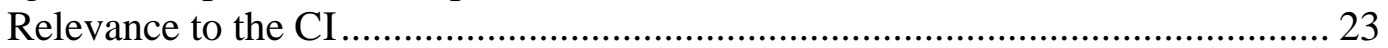

The CI: Linguistic/Cognitive Complexity and Social Support................................ 23

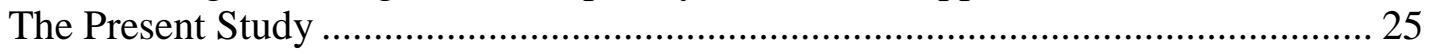

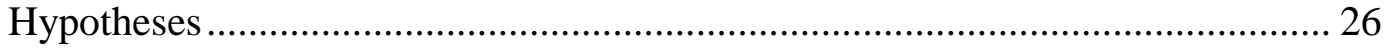

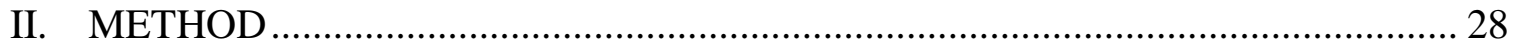

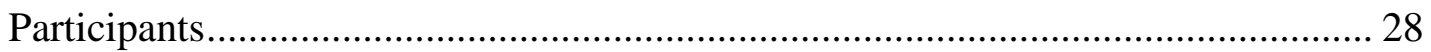

Native English speakers ................................................................................ 29

Non-native English speakers....................................................................... 29

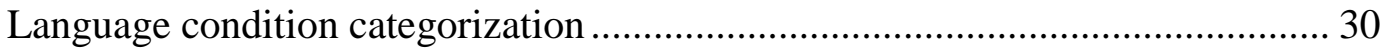

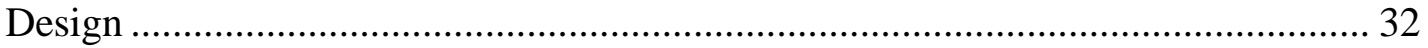

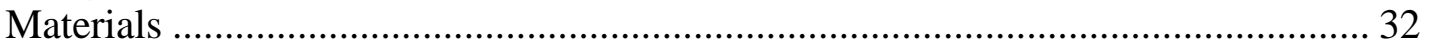

Language History Questionnaire ................................................................. 32

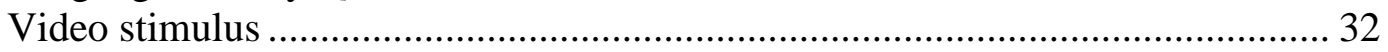

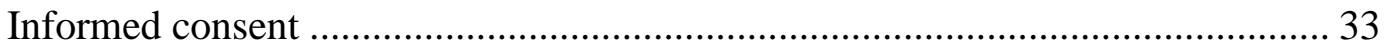

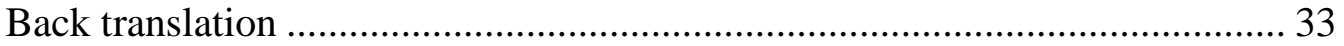

Research Assistant Training ……………………………………………...... 33

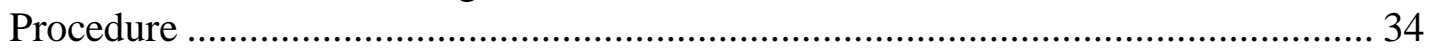

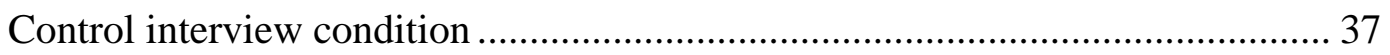

Reverse order interview condition .................................................................... 38

Transfer of control interview condition ........................................................... 38

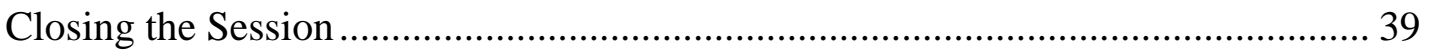

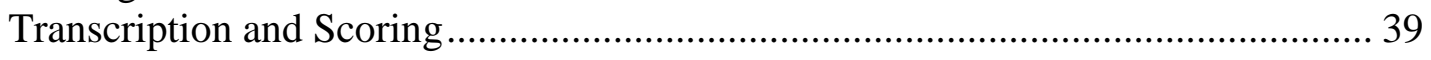

Inter-rater Reliability ………………………………………........................... 41

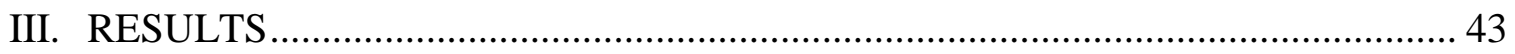

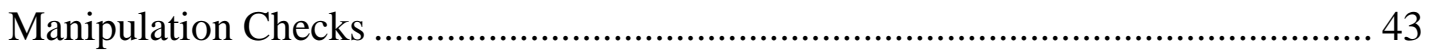

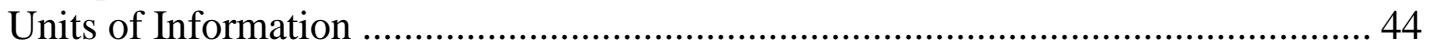

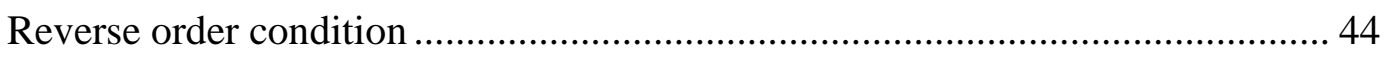

First recall attempt only .............................................................................. 46

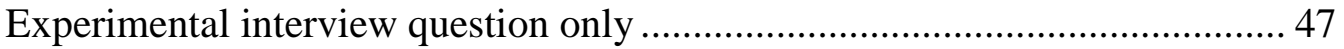

Transfer of control condition .......................................................................... 49

Accuracy of Reported Details ............................................................................ 51 


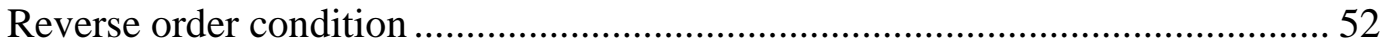

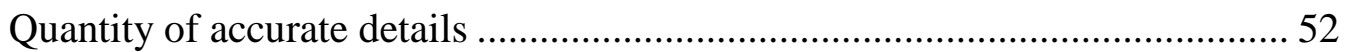

Proportion of accurate details ..................................................................... 54

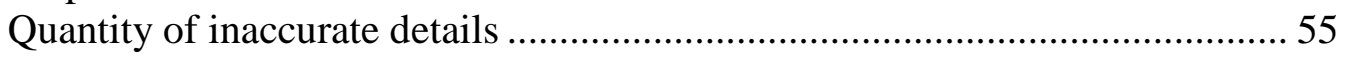

Proportion of inaccurate details ................................................................... 55

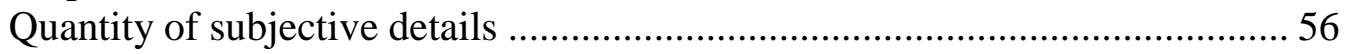

Proportion of subjective details ................................................................ 57

Quantity of non-scoreable details .................................................................. 59

Proportion of non-scoreable details ............................................................. 59

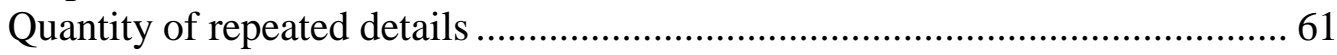

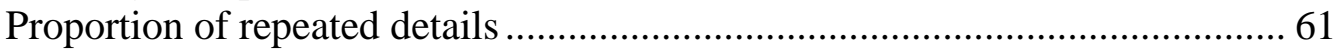

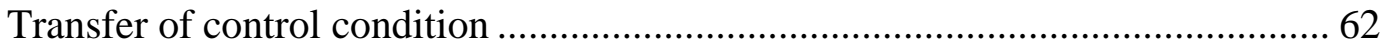

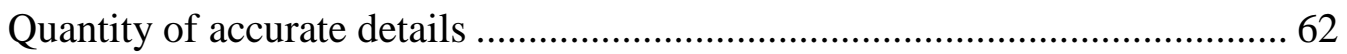

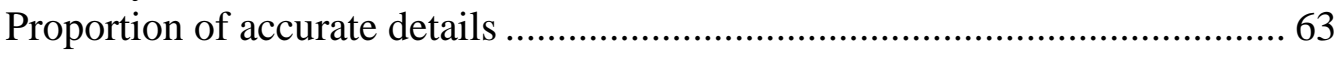

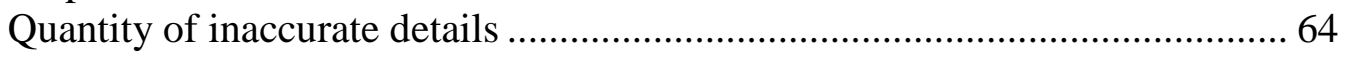

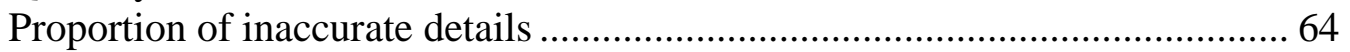

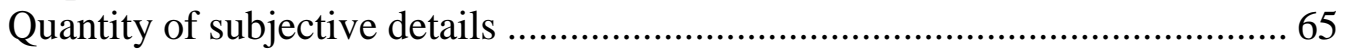

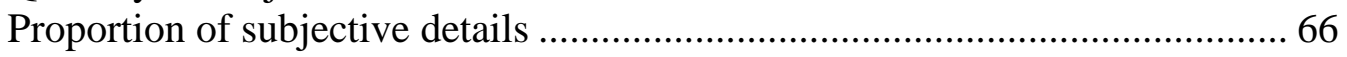

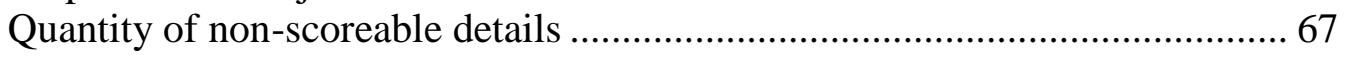

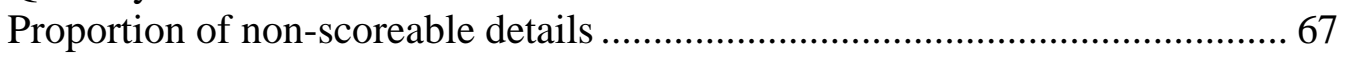

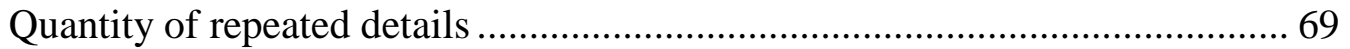

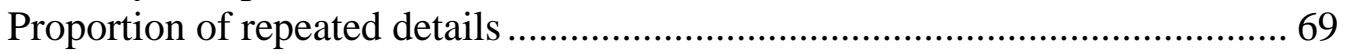

Accuracy Rates Across Interview Questions ........................................................ 71

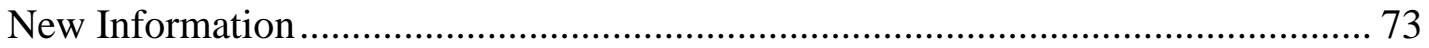

English Proficiency and Quantity of Detail ............................................................ 74

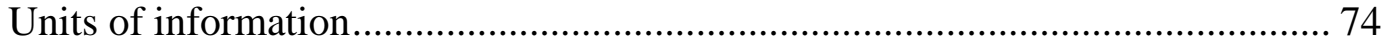

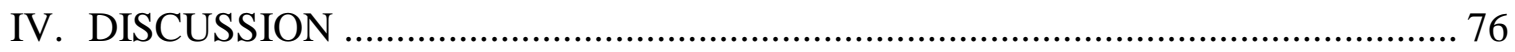

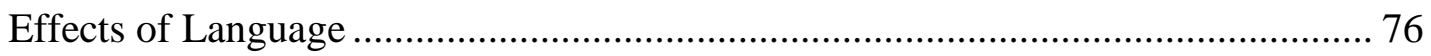

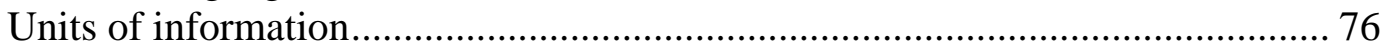

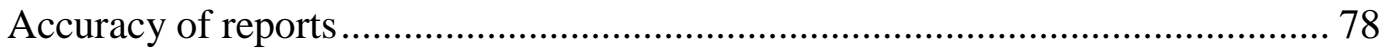

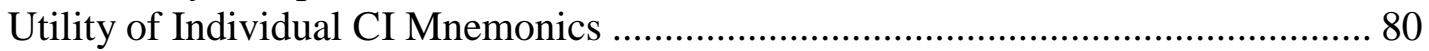

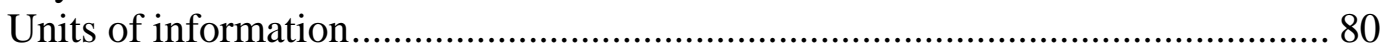

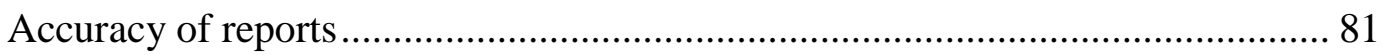

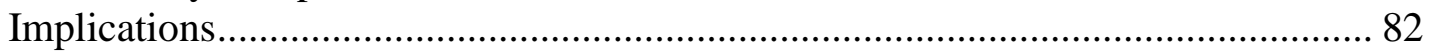

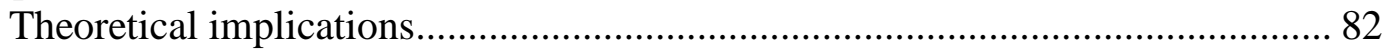

Practical implications................................................................................. 84

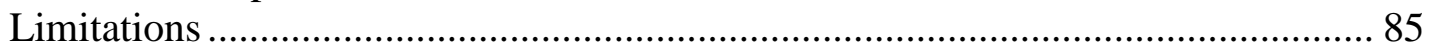

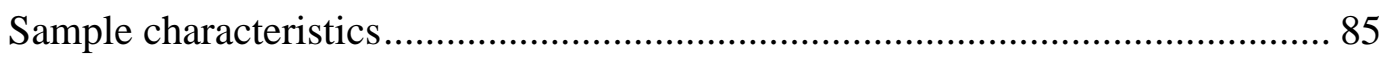

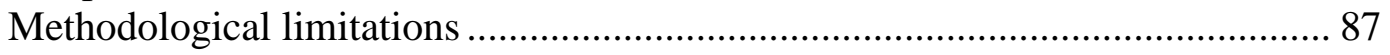

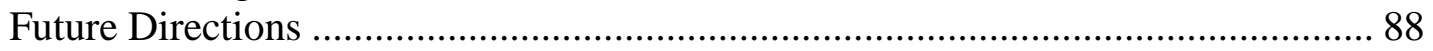

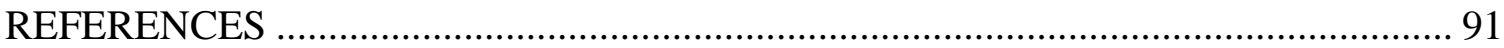

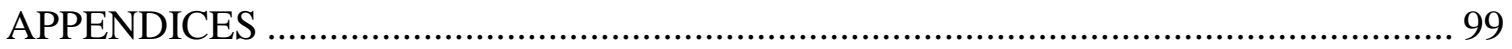




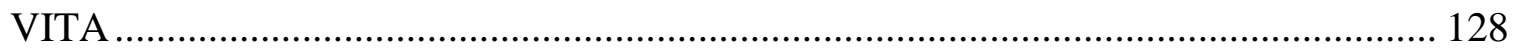




\section{LIST OF TABLES}

TABLE

PAGE

Table 1. First languages reported by NNES participants

30

Table 2. Participant self-ratings of English ability (Language History Questionnaire,

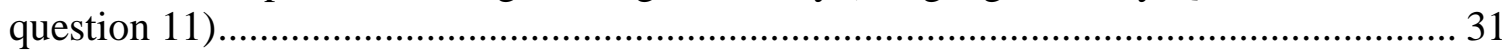

Table 3. Intraclass correlation coefficients for detail scores......................................... 42

Table 4. Estimated marginal means and standard deviations for reverse order

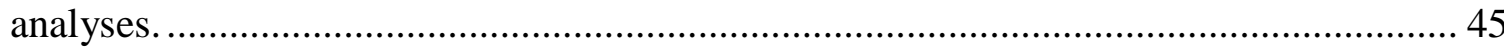

Table 5. Estimated marginal means and SDs for transfer of control condition ............... 51

Table 6. Descriptive statistics for accurate details (control vs. reverse order

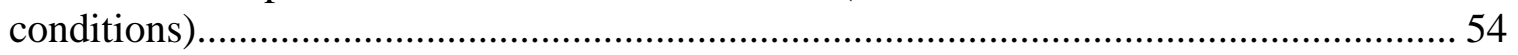

Table 7. Descriptive statistics for inaccurate details (control vs. reverse order conditions)

Table 8. Descriptive statistics for subjective units (control vs. reverse order conditions)

Table 9. Descriptive statistics for non-scoreable units (control vs. reverse order conditions)

Table 10. Descriptive statistics for repeated units (control vs. reverse order conditions)

Table 11. Descriptive statistics for accurate details (control vs. transfer of control conditions).

Table 12. Descriptive statistics for inaccurate details (control vs. transfer of control conditions).

Table 13. Descriptive statistics for subjective details (control vs. transfer of control conditions)

Table 14. Descriptive statistics for non-scoreable details (control vs. transfer of control conditions).

Table 15. Descriptive statistics for repeated details (control vs. transfer of control conditions).

Table 16. Descriptive statistics for between-participants analysis of proportion of accurate details (control vs. reverse order condition). 
Table 17. Descriptive statistics for quantity of new details reported to the second

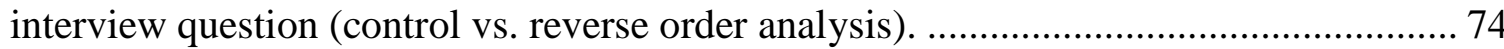

Table 18. Correlations between participants' reported English proficiency and

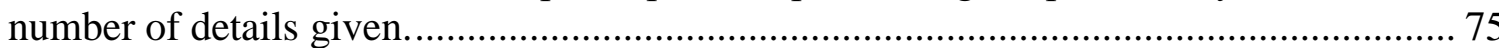




\section{LIST OF FIGURES}

\section{FIGURE}

PAGE

Figure 1. Levelt's speech production model (Levelt, 1989; Levelt, Roelofs, \& Meyer, 1999)

Figure 2. Word association hypothesis (Potter, So, Von Eckardt, \& Feldman, 1984) ..... 15

Figure 3. Concept mediation hypothesis (Potter, So, Von Eckardt, \& Feldman, 1984)... 15

Figure 4. Revised hierarchical model (Kroll \& Curley, 1988) ..................................... 17

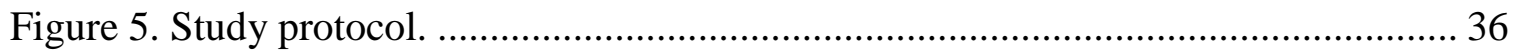

Figure 6. Estimated marginal means and 95\% confidence intervals for units of information reported across both interview questions (control vs. reverse order conditions).....

Figure 7. Estimated marginal means and $95 \%$ confidence intervals for units of information reported during the first interview question (control vs. reverse order conditions)....

Figure 8. Estimated marginal means and $95 \%$ confidence intervals for units of information reported to the experimental interview question (control vs. reverse order conditions).

Figure 9. Estimated marginal means and 95\% confidence intervals for units of information reported in the first interview question (control vs. transfer of control conditions).....

Figure 10. Estimated marginal means and 95\% confidence intervals for units of information reported to the second (experimental) interview question (control vs. reverse order conditions).

Figure 11. Estimated marginal means and 95\% confidence intervals for proportion of subjective units of information reported to the second interview question (control vs. reverse order conditions)......

Figure 12. Estimated marginal means and 95\% confidence intervals for proportion of repeated details reported in the first interview question (control vs. transfer of control conditions)

Figure 13. Crime scene from stimulus video.

Figure 14. Perpetrators from stimulus video. 


\section{ABBREVIATIONS AND ACRONYMS}

Analysis of variance

ANOVA

Cognitive interview

CI

Complexity, accuracy, and fluency

CAF

English Language Institute

ELI

Intraclass correlation

ICC

First language

L1

Second language

L2

Language History Questionnaire

LHQ

Mean

M

Non-native English speaker

NNES

Standard deviation

SD 


\section{INTRODUCTION}

In the United States, 61.8 million residents report that they speak a language other than English in their homes; over 25 million of these residents (over 40\%) report that they speak English "less than very well" (Camarota \& Zeigler, 2014). Non-native English speakers (NNESs) $)^{1}$ represent a sizeable proportion—approximately $8 \%$ - of the United States population, and may be victims of or eyewitnesses to crimes, requiring them to provide statements to police investigators. Though some of these individuals may be provided with interpreters, others may need to provide a statement in English, i.e., their second language (L2). Research on police perceptions of interpreters from Australia and from the United States indicates that police are least likely to seek interpreters when interviewing witnesses compared to interviewing victims or suspects (Shaffer \& Evans, 2018; Wakefield, Kebbell, Moston, \& Westera, 2015). Furthermore, there may also be jurisdictions or situations with limited access to interpreters (Rivera \& Zraick, 2010). Thus, interpretation may not seem necessary or may not be immediately availablemeaning the interview occurs in English.

Though there are best practice witness interviewing guidelines, relatively little research has focused specifically on interviewing NNES witnesses. The lack of research with NNES samples is concerning given that NNES witnesses may be interviewed in English, a language in which they may not be proficient. Interviewing eyewitnesses who are not proficient in the language may adversely affect the amount of details or the

\footnotetext{
${ }^{1}$ The phrase "non-native English speakers" may have enthnocentric connotations in some fields. However, it is used in the present dissertation to be consistent with the most closely related scientific literature, i.e. lie detection among non-native English speakers.
} 
reliability of details provided by the witness. The current study aims to test the utility of recommended interviewing methods among NNES witnesses.

\section{The Cognitive Interview}

One of the most commonly recommended techniques for interviewing witnesses is the cognitive interview (CI; Fisher \& Geiselman, 1992). The CI refers to several investigative interviewing techniques and mnemonics that are designed to maximize the number of details elicited by eyewitnesses and victims. The CI was developed with an eye toward (a) the social dynamics of interviews, (b) effective communication between witnesses and interviewers, and (c) the cognitive processes of memory. Some of these CI considerations are discussed below.

Social factors. A CI often begins with building rapport, which helps the witness become comfortable with the interviewer. Broadly, rapport refers to an interpersonal relationship between the interviewer and the witness (Vallano \& Schreiber Compo, 2015). Establishing an interpersonal connection can help overcome barriers to reporting or disclosure on the witness's part. Many interviews require witnesses or victims to recount personal, unpleasant, and perhaps traumatic events to strangers; these strangers are often armed, uniformed police officers, who can seem intimidating (Fisher, Milne, \& Bull, 2011). Interviewers can begin by asking questions that uncover shared values or experiences to create a comfortable atmosphere for the witness. A comfortable atmosphere helps the witness feel at ease reporting intimate details. Building rapport has been shown to increase the amount of correct information provided by witnesses, in response to both free recall questions and cued follow-up questions (Collins, Lincoln, \& Frank, 2002). 
Interviewers also emphasize to the witness that his or her role is an active one: to provide as much detailed information as possible without waiting to be specifically prompted (Fisher \& Geiselman, 2010). Taking such an active role is often in contrast to witnesses' expectations of an interview (e.g., they often assume that the interview will follow a strict question-answer pattern, led by the interviewer). The interviewer should primarily ask open-ended questions that encourage the witness to generate as much detail as possible.

Additionally, the interviewer can provide a transfer of control instruction, which emphasizes that the witness is the expert in the current situation (Fisher \& Geiselman, 1992). The transfer of control instruction also sets the social dynamic such that the witness - not the interviewer - should do most of the talking during the interview. Interviewers can convey the transfer of control instruction to witnesses by emphasizing that the witness knows the relevant information whereas the interviewer does not. For instance, if a witness saw a robbery, the interviewer could say to the witness, "I was not there [at the scene of the robbery], you were; you know what happened, so I need you to tell me everything you can remember." The transfer of control instruction can also communicate to witnesses that they are free to report their memories in whichever manner or order they wish (Paulo, Albuquerque, \& Bull, 2013). Although the transfer of control instruction is sometimes included in research using full CI protocols - especially studies testing the enhanced CI-research on the effects of the instruction specifically is scant.

Effective communication. Communication is obviously a key component of investigative interviews: police officers must communicate their needs, and witnesses 
must communicate their memories. A major concern is that witnesses often fail to report details that seem (to the witness) to be inconsequential. However, seemingly inconsequential details can be critically important for police investigators. Moreover, recall of these seemingly inconsequential or partial details can help cue retrieval of additional, relevant details. Therefore, an important explicit instruction given to witnesses is that they should report everything they remember about the event (Fisher \& Geiselman, 2010). The "report everything" instruction helps encourage witnesses to output the level of detail required by investigators to conduct the investigation.

As with other instructions, there are many ways to instantiate the effective communication principle in a CI. One other method draws upon the cognitive principle of transfer appropriate processing (Morris, Bransford, \& Franks, 1977). Essentially, the most effective way to retrieve a memory is in the same format as the memory was encoded. As such, verbal reports are sometimes not the most effective way to report memory. Instead, interviewers might ask witnesses to sketch the layout of the crime scene, or demonstrate the perpetrator's gait, or use other forms of nonverbal output (Leibowitz, Guzy, Peterson, \& Blake, 1993).

Establishment of these social dynamics and guidelines for effective communication typically occur toward the beginning of the interview. During the introductory phase of the interview, the investigator should build rapport with the witness, transfer control to the witness (e.g., "you are the expert-you know what happened, and I don't'), emphasize active witness participation (e.g., "I want you to do most of the talking, and I will be mostly taking notes"), and instruct the witness to report everything s/he can remember (e.g., "every detail is important, so you should tell me 
everything you can remember, even if it seems irrelevant”). Importantly, witnesses should also be instructed not to guess. These instructions comprise an introductory phase of the CI that helps orient witnesses to the goals of the interview and helps them overcome social hurdles to providing extensive, detailed accounts (Geiselman \& Fisher, 2014).

Cognitive processes. Cognitive interview mnemonics, like context reinstatement or the reverse order technique, are constructed from theoretical cognitive processes that underlie human memory. Investigators will elicit lengthy, detailed reports from witnesses if they take advantage of basic memory processes. For example, a well-established principle in cognitive psychology is the encoding specificity principle. In short, the encoding specificity principle states that memory content is encoded along with context (Tulving \& Thomson, 1973). Thus, people's ability to retrieve information is best when their context at retrieval matches the context in which they encoded the information. Reinstatement of context can provide retrieval cues that help the participant (or witness) to access details stored in memory. A classic experiment by Godden and Baddeley (1975) demonstrated the benefit of context reinstatement by having scuba divers learn a list of words either on dry land or underwater, and then testing the divers either on dry land or underwater. Participants' recall for the list of words was best when the learning condition and retrieval condition matched (i.e., learn and retrieve underwater or learn and retrieve on dry land). Typically, context reinstatement is instantiated in the CI as an instruction for witnesses to mentally recreate their context at the time of the crime. For example, interviewers may ask witnesses to recall their thoughts and feelings at the time of the crime. By mentally reinstating the context of the crime-i.e., what the witness was 
seeing, hearing, feeling, thinking, etc. - - the witness can recall more details from that episode.

Another basic cognitive principle is reminiscence: people often do recall previously unrecalled details when they perform follow-up memory searches (Ballard, 1913). Reminiscence can lead to hypermnesia — an increase in recalled details in a second recall attempt as compared to an initial recall attempt (Payne, 1987; Roediger \& Payne, 1982). Specifically, hypermnesia occurs when the number of newly recovered details during later retrieval attempts exceeds the number of forgotten details. In other words, more details are retrieved during retrieval attempt \#2 than during retrieval attempt \#1. While it seems suspect that a witness can suddenly recall new, previously unreported information in response to a follow-up question, many studies demonstrate such reminiscence (Bornstein, Liebel, \& Scarberry, 1998; Eugenio, Buckhout, Kostes, \& Ellison, 1982). A series of experiments by Turtle and Yuille (1994) found evidence for reminiscence but not hypermnesia within eyewitness experimental paradigms. Though their participant-witnesses did recall previously unreported details during successive retrieval attempts, they also failed to report (forgot) previously mentioned details; the retrieval of previously unreported details demonstrates reminiscence, but the forgetting of previously reported details resulted in no net gain for number of details in the second recall attempt (thus, no hypermnesia).

The CI takes advantage of reminiscence (and potentially hypermnesia) by encouraging multiple and varied retrieval attempts. There are numerous ways to implement multiple and varied retrieval attempts, but the reverse order and change 
perspective mnemonics are specific examples included in the original CI (Fisher \& Geiselman, 1992).

The reverse order technique requires witnesses to recount events backward, starting with the end of the event. For example, if witnesses were interviewed about a bank robbery, they may begin by recounting how the thief exited the bank. Bellezza and Bower (1982) argue that activated schemas serve as memory cues for recalling sequences of events; as a result, witnesses/participants are more likely to recall schema-consistent events compared to unusual or schema-inconsistent events. However, unusual or schemainconsistent events can be relevant for criminal investigations. Asking witnesses to recount their memories in reverse order helps them to search their memory "frame by frame" instead of relying on a script or schematic reconstruction (Geiselman, Fisher, MacKinnon, \& Holland, 1986). In an experiment specifically testing reverse order recall, Geiselman and Callot (1990) presented participants with two stories embedded with (schema) consistent actions and incidental actions. Participants recalled the events either in forward order or in reverse order. Results showed that participants who recalled the story in forward order reported mostly schema-consistent actions, whereas participants who recalled the story in reverse order reported mostly incidental actions. The reverse order instruction can help witnesses uncover incidental or non-schematic details, which can be useful to investigators.

The change perspective mnemonic requires witnesses to recall the event from another person's point of view (for example, a co-witness or the perpetrator). Using the change perspective technique, our bank robbery witness might recount the robbery from the thief's perspective. Anderson and Pichert (1978) conducted a classic study employing 
a change perspective instruction. Participants read a story about a house from either a burglar's perspective or a homebuyer's perspective. Afterward, they were asked to recall as much of story as possible (from the story's original perspective); then, they were either asked to recall the story again with no change in perspective or to recall the story again from a new perspective. For example, the researchers may have asked a participant who initially read the story from the homebuyer's perspective to recall the story from the burglar's perspective later. The results showed that when participants changed perspectives, they were able to recall new, previously unreported details that were relevant to the new perspective. For instance, a participant who read the story from the burglar's perspective was only able to recall a detail about the leaky roof when recalling from the homebuyer's perspective. In an eyewitness context, recalling the scene of the crime from another perspective (e.g., the criminal's perspective or another bystander's perspective) can make important_-but previously unreported—details salient.

Gilbert and Fisher (2006) combined these mnemonics to test for reminiscence (and hypermnesia). Participants first viewed a video-recorded bank robbery as the to-beremembered stimulus. Participants were randomly assigned to a free recall, chronological order, reverse order, police perspective, or witness perspective retrieval condition during an initial interview and were again randomly assigned to one of the five conditions during a follow-up interview 48 hours later. Nearly all of the participants $(98 \%)$ recalled at least two reminiscent details during the second interview-i.e., new details that were not reported during the first interview. The number of reminiscent details was significantly higher when the retrieval cues changed from the first to the second interview. For example, participants recalled more previously unreported details when they were first 
interviewed with a change order instruction and later interviewed with a policeperspective instruction compared to participants who were interviewed with, e.g., the police-perspective at both retrieval attempts. These findings indicate that probing witnesses' memories using different retrieval cues allows them to access new, previously unreported details. Furthermore, these instructions elicited more details than a simple free recall instruction. These findings lend further support to the use of reverse order and change perspective CI mnemonics.

Empirical support for the CI. Typically, the CI is very effective; two metaanalyses of the CI literature show that use of the CI yields substantially more correct details compared to a standard, comparison interview $(d \geq 0.80)$ (Köhnken, Milne, Memon, \& Bull, 1999; Memon, Meissner, \& Fraser, 2010). Though both meta-analyses also indicate small increases in the number of incorrect details reported, Köhnken and colleagues' meta-analysis did not find a change in overall accuracy rates (overall accuracy was not reported by Memon and colleagues). However, although over 50 studies demonstrate the effectiveness of the CI, relatively few address which of the component elements most strongly drive the effect.

The few studies that investigate isolated CI mnemonics have yielded mixed results. One experiment tested various combinations of CI mnemonics (Boon \& Noon, 1994). In the Boon and Noon (1994) study, participants initially watched a videotaped robbery as the to-be-remembered stimulus. Then, participants were asked a free recall question with the instruction to "report all." The time 2 interview was randomly assigned to be a reverse order mnemonic, a change perspective mnemonic, a context reinstatement mnemonic, or a "try again" prompt. Additionally, there was a control group who was 
initially read a set of standard instructions (instead of the "report all" instruction) and received the "try again" prompt at time 2 . The study demonstrated the relative benefit of all CI mnemonics compared to the control condition except the change perspective mnemonic. The change order, reinstate context, and "report all" plus try again conditions all elicited significantly more accurate details at time 2 compared to the change perspective and control conditions, which did not differ from each other. Another study also sought to test each of these four original CI mnemonics in isolation in both adult and child samples (Milne \& Bull, 2002). Milne and Bull's (2002) study compared performance across the four mnemonics (plus one condition combining the report everything and context reinstatement instructions) to a control condition wherein participants were asked a free recall question and then were simply told to "try again." Milne and Bull found, in adult participants, an increase in details reported after the combined $\mathrm{CI}$ mnemonics condition relative to most conditions isolating $\mathrm{CI}$ mnemonics (e.g., receiving only the reverse order instruction) and relative to the control, "try again" condition. Among the adult participants, the number of details recalled in the combined condition did not differ significantly from the context reinstatement condition; the number of details recalled in each of the isolated CI conditions did not differ significantly from each other. In sum, the combined condition and context reinstatement condition were superior to all the isolated mnemonic conditions. A major limitation of Milne and Bull's study is sample size: with 125 participants total (and only 34 in the adult age group across six between-participant conditions), it is possible that the study was underpowered to detect differences within each age group. 
An additional study reported by Milne and colleagues compared college students' memory performance across the four original CI mnemonics (Memon, Cronin, Eaves, \& Bull, 1996). In the study, a graduate student interrupted a large lecture class, and the undergraduates were interviewed as witnesses 1-2 weeks later. All student-witnesses were asked an initial free recall question and then one of four experimental questions: change perspective, change order, reinstate context, or try again (control). Though the total number of details reported did not differ across conditions, the types of details reported did differ. The change order condition was especially helpful in eliciting temporal details, and the change perspective condition was especially helpful in eliciting location information. Further research with a large sample can help elucidate which CI mnemonics are most helpful.

Despite the little research on the Cl's original mnemonics, some additional mnemonics have been tested in isolation. Asking witnesses to close their eyes, for example, has consistently been found to increase the number of correct details reported by participants (Vredeveldt, Baddeley, \& Hitch, 2013; Vredeveldt \& Penrod, 2013). Asking witnesses to sketch while narrating has also been shown to increase correct detail recall in both adults (Dando, Wilcock, \& Milne, 2008) and children (Otgaar, van Ansem, $\&$ Pauw, 2016). These findings show support for the efficacy of some cognitive mnemonics when isolated, yet previous research on the original mnemonics is less clear.

It is important to determine which CI mnemonics are most effective because a number of studies indicate that police investigators often use truncated or altered versions of the $\mathrm{CI}$ in real investigations. If investigators are going to select only one or two $\mathrm{CI}$ mnemonics to use, then it is important to know which mnemonics contribute most to 
witnesses' recall and which are less essential. For example, a sample of British police investigators reported that rapport building, uninterrupted recall, and "report everything" instructions were among the most frequently used CI mnemonics, whereas the change order and change perspective mnemonics were less frequently used (Dando, Wilcock, \& Milne, 2008). Despite being used less frequently than other mnemonics, $30 \%$ of all respondents reported using the change order mnemonic to be "quite effective." Thus, these mnemonics are occasionally used, and some investigators believe that they are quite effective.

\section{Language, Cognition, and Memory}

As mentioned, there is a sizeable percentage of NNES residents in the United States who may be eyewitnesses; these NNES witnesses will likely be interviewed, and these interviews may contain CI mnemonics. Despite the CI's record for increasing output with no cost to accuracy, it is unclear whether the effect is present for NNES witnesses, a group that has received little-to-no attention in the witness interviewing literature. Witnesses who are NNESs face some specific cognitive and linguistic challenges that may impede their responses to typical CI mnemonics.

Speech production can generally be characterized as having three stages: conceptualization, formulation, and articulation (Levelt, Roelofs, \& Meyer, 1999; see Figure 1). The conceptualization stage consists of the speaker retrieving relevant information from long-term storage. In a witness interviewing context, the speaker must recall the episodic event of interest. The formulation stage requires the speaker to choose words that express the concepts from the previous stage; essentially, the speaker creates an internal speech or a plan for what s/he will say. In a witness interviewing context, the 
formulation stage consists of translating the episodic information into verbal information. Finally, the articulation stage is the physical execution of the speech constructed in the formulation stage. In other words, the speaker produces the speech.

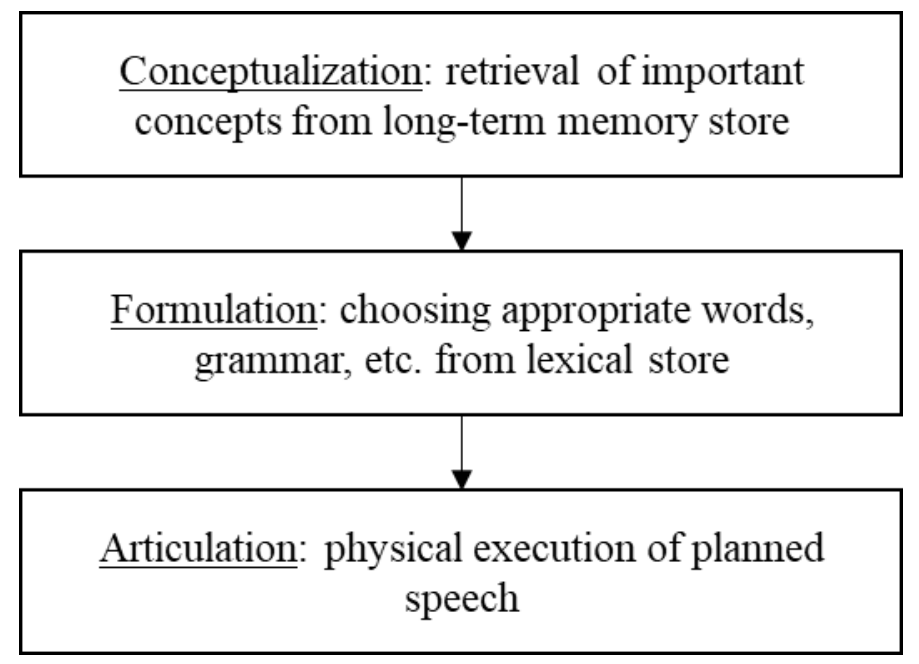

Figure 1. Levelt's speech production model (Levelt, 1989; Levelt, Roelofs, \& Meyer, 1999)

Formulation and articulation are thought to be relatively automatic processes in fluent speakers (Levelt, 1989). With extensive experience in a language, speakers typically do not need to devote attentional resources to choosing precise words and correct grammatical constructions. Speakers also do not typically need to devote attention to motor functions (e.g., tongue placement, mouth shape) to create their intended messages. According to the speech production framework, the most cognitively demanding aspect of communication is conceptualizing the message. Speakers do spend cognitive resources planning the meaning of their messages, but constructing and 
speaking typically do not require conscious, attentional intervention when a speaker has extensive experience in the language (e.g., a speaker's native language).

Speaking in a second, non-native language, however, is more cognitively taxing than speaking in a first language (Ardila, 2003). Again, according to Levelt's model, conceptualization of the message requires attention. However, unlike with fluent speakers, for NNESs, formulating the message with appropriate lexical content and grammatical structure also requires attention. Because speakers have less experience and practice producing speech in their non-native language, their non-native language is more difficult to access compared to their native language (Kapatsinksi, 2010). Potter, So, Von Eckardt, and Feldman (1984) proposed and tested two models to explain how lexical representation (language) is related to conceptual representation (memory).

The first of these models, the word association model, proposes that words in a speaker's non-native language are linked to words in the speaker's native language (see Figure 2). Words in the speaker's native language are directly associated with the speaker's conceptual store (memory). So, for example, a Spanish speaker who learns English as a second language would first access his/her conceptual store, find the Spanish word that matches the concept (e.g., "árbol”), and then find the English word that matches the Spanish word (e.g., "tree"). The word association hypothesis suggests that NNESs formulate their conceptualized message in their native language, and then translate that message into their non-native language. 


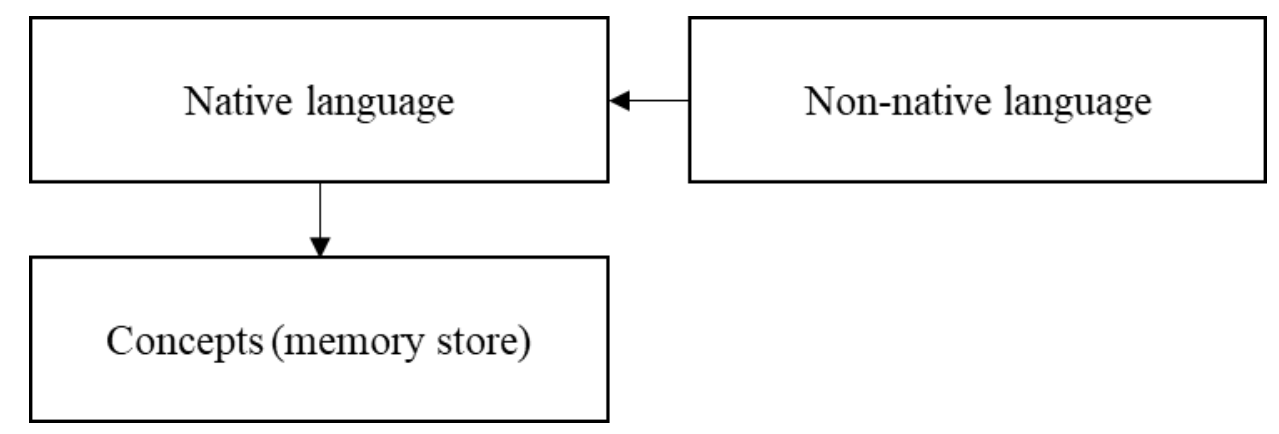

Figure 2. Word association hypothesis (Potter, So, Von Eckardt, \& Feldman, 1984)

Another model, the concept mediation model, proposes that words in a speaker's native and non-native language are not associated with each other but rather with an underlying conceptual store (see Figure 3). So, a Spanish speaker who learns English as a second language would identify the concept in their long-term or semantic storage (e.g., a large plant with a trunk and leaves), and then search for the appropriate word in his/her non-native language store (e.g., "tree"; the Spanish word "árbol” is never activated). Since the non-native language is used less frequently than the native language, accessing the non-native language is more effortful than accessing the native language.

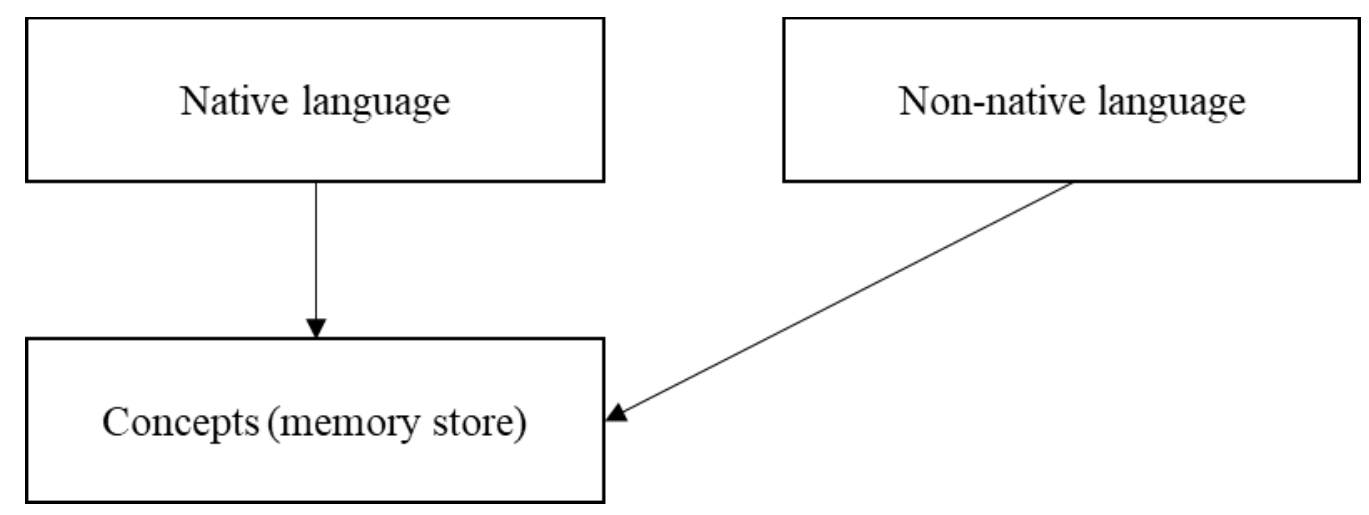

Figure 3. Concept mediation hypothesis (Potter, So, Von Eckardt, \& Feldman, 1984) 
These models imply specific predictions. The word association model suggests that direct translation tasks should occur more quickly than identification of pictures in a second language (e.g., a speaker should be quicker to translate "árbol" as "tree" than to identify a picture of a tree). The conception mediation model suggests the oppositeidentification of concepts should occur more quickly than direct translation tasks. Potter and colleagues' (1984) results from both highly proficient and less-proficient samples supported the concept mediation hypothesis. However, research by Kroll and Curley (1988) suggests that novice NNES - those with two years or fewer of experience with the language - were faster at direct translation tasks than picture naming tasks. They suggest that word association is indeed a step toward learning a non-native language, but speakers begin to associate L2 words with concepts rather than L1 words as they become more proficient. They offer a revised hierarchical model that combines aspects of both the word association and concept mediation models (see Figure 4). Specifically, they posit a developmental shift from the word association model to the concept mediation model. As a speaker increases in non-native language proficiency, s/he will increasingly access L2 words directly instead of translating from L1 to L2. Despite a shift in reliance between languages, highly proficient non-native speakers sometimes still rely on lexical associations between L1 and L2 (Menenti \& Indefrey, 2006). 


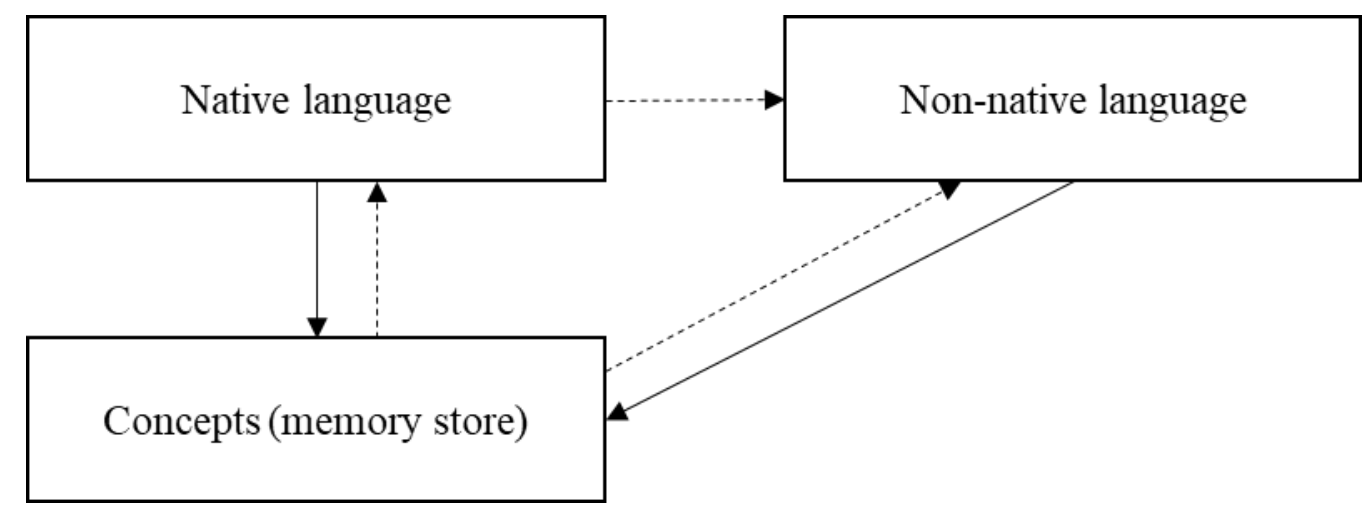

Figure 4. Revised hierarchical model (Kroll \& Curley, 1988)

In addition to these models of speech production, there are also theoretical models of bilingual (multilingual) memory representation. Dual-coding frameworks of memory suggest that human memory consists of a visual memory plus a verbal store (Paivio, 1979). For bilingual individuals, it has been proposed that separate verbal stores exist for each language, each of which is linked to images in the visual memory store (Paivio \& Desrochers, 1980). Dual-coding frameworks are similar to the concept mediation model of speech production discussed above. However, whereas the models above focus primarily on production of speech, dual-coding models attempt to explain how language influences memory encoding and retrieval processes. Paivio and Lambert (1981) developed a classic paradigm to test dual-coding theories. In their paradigm, participants are given an image, a French word, or an English word and are asked to write the corresponding English word (i.e., name the image, translate the French word, or copy the English word). Participants were then asked to freely recall all the English words. Results showed approximately a 3:2:1 ratio, such that participants were about three times more likely to recall a word generated for an image compared to an English word copied, and 
about twice as likely to recall a translated word compared to a copied English word. Jared and colleagues (2013) recently replicated these findings in a sample of Mandarin-English bilingual participants. They also found that images from Chinese culture (e.g., a Chinese mailbox, which is cylindrical) are named more quickly in Mandarin than English, and vice versa for images from Western culture (e.g., a Canadian mailbox, which is boxy). These results suggest that there are links between the visual store and two separate language systems. That is, retrieval seems to be best when information is encoded on the basis of the underlying conceptual or visual representation of that item.

An additional concern — as well as a factor requiring attention dispersalregarding L2 production is a social one: Though a speaker might be able to produce speech in his/her non-native language, s/he may not feel comfortable doing so. MacIntyre and colleagues (1998) describe a heuristic model of variables that influence speakers' willingness to communicate in their non-native language. Some of the variables, like communicative competence for instance, induce a relatively stable effect on speakers' willingness to communicate. Speakers who assess their competences as being relatively low will frequently be hesitant to communicate (Celce-Murcia, Dörnyei, \& Thurrell, 1995). However, many of the variables are context dependent. Various situational factors like proficiency of the conversational partner, physical setting, goals, desire to communicate with a specific person, and self-confidence (in the moment) are context dependent. These situational factors are fluid and can motivate or de-motivate a nonnative speaker to communicate. Proficiency of the conversational partner can also influence how non-native speakers engage in conversation: when the partner is a native speaker, non-native speakers tend to be passive and avoidant (Hatch, 1992). 
Additionally, interactions can take place in different physical and social contexts: a non-native speaker may be very confident communicating in an academic setting (e.g., a classroom) but less confident communicating information in a legal setting (e.g., a police station). Furthermore, non-native speakers may be more competent achieving certain communicative goals in a non-native language compared to other goals; a speaker may be better able to describe personal information in a non-native language than to persuade another person. Desire to communicate with a specific person may stem from the non-native speaker's perception of being in the same "ingroup" as the partner (Clément, 1980). Finally, state-dependent self-confidence is thought to be driven by perceived confidence in general and level of anxiety in the moment (Clément, 1980); speakers who are anxious may be unwilling to communicate in a non-native language, even if they are relatively proficient. Conversational partners can increase a NNES's willingness to communicate by leveraging these social determinants of willingness. In the case of an eyewitness interview, police officers could draw an interpersonal connection with the NNES witness, emphasize the specific goal (communicate memory), attempt to soothe situational anxiety, and attempt to bolster the witness's confidence; these may help NNES be more willing to communicate in an unfamiliar context (i.e., a police interview).

\section{Investigative Interviewing of Non-Native Speakers}

A limited body of research has examined investigative interviewing of NNES participants. Some of the literature focuses on interviewing witnesses, but most of the literature focuses on interviewing suspects or on human intelligence gathering. Suspect and intelligence gathering interviews can inform the current study because they often 
include innocent experimental conditions and relevant outcome measures (e.g., number of details). These conditions are most similar to the current research, which focuses on witness memory. Some of these studies specifically examine the role of interpreters in these interactions; those that include an interpreter-absent condition can also inform the current study. A discussion of investigative interviewing research with NNESs as participants follows.

A recent study specifically investigated NNESs' performance in investigative interviewing (Martin, Evans, \& Baralt, in preparation). After viewing a mock crime, native English speakers and native Spanish speakers were interviewed in English. In response to open-ended free recall questions, there were no differences in the number of details reports by NNESs versus native speakers; however, NNESs provided fewer accurate details than native speakers did.

Though there is a little research on interviewing NNES-witnesses per se, some of the research on the effects of interpreters in investigative interviews includes a noninterpreter condition; the results from these conditions are also relevant to discuss here. A study by Ewens and colleagues (2016a) randomly assigned native English speakers and NNESs (through an interpreter or not) to either tell the truth or lie about their jobs. Comparisons between native English speakers and NNES (without an interpreter) telling the truth can inform the present line of research. Ewens and colleagues (2016a) found that, compared to participants interviewed in their native language, participants interviewed in their non-native language provided fewer details overall. Similarly, in another study, Ewens and colleagues (2016b) gave participants a model statement to convey the level of detail desired by the interviewer. Participants were native English 
speakers, NNESs interviewed in English, or NNESs interviewed through an interpreter; participants watched a video of a secret meeting and were randomly assigned to either tell the truth or lie about it. Participants first gave a free recall, then heard the model statement, then gave a second free recall. From Ewens and colleagues' (2016b) study, comparisons between the native English speakers and NNESs (with no interpreter) in the truthful condition are most relevant to the current research. Reports in the first free recall showed that native English speakers provided more details than NNESs. Furthermore, giving participants a model statement (an example of the level of detail desired by the interview) resulted in more details reported by native speakers but no increase in details reported by NNESs.

In addition to the number of details reported overall, accuracy of NNESs' reports may be a concern. In a series of studies testing the misinformation effect in bilingual participants, Shaw, Garcia, and Robles (1997) presented participants with a videotaped mock crime (no language component), post-event misinformation, and a follow-up test. The post-event misinformation was presented in either Spanish or English, and the follow-up test was present in either Spanish or English. The results showed a misinformation effect (i.e., participants reported having seen details in the mock crime that were only mentioned by the researcher later)—consistent with classic literature on the topic (for a review, see Loftus, 2005). The misinformation effect was observed in the language-consistent conditions (i.e., English-English and Spanish-Spanish) as well as the cross-language conditions (i.e., Spanish-English and English-Spanish). Shaw and colleagues' studies suggest that misinformation is robust to language condition and may influence the conceptual or visual trace of the stored memory. 
These studies show that witnesses who are interviewed in a non-native language provided fewer details overall than witnesses interviewed in their native language. Some interviewing mnemonics like the model statement are not effective at increasing detail output from non-native speakers. Moreover, NNESs are just as vulnerable to the misinformation effect as native English speakers. The present review of the literature highlights the need for research on interviewing techniques that increase the number of details communicated by NNES witnesses while being careful to avoid misinformation.

\section{Linguistic Properties of Complex Tasks}

Cognitive resource availability is a critical concern when interviewing NNESs because they are already engaged in a cognitively demanding task, i.e., speaking a second language (Ardila, 2003). Adding a second cognitively demanding task, like manipulating retrieved memories via the reverse order or change perspective technique, divides cognitive resources between two complex tasks, which may result in performance decrements in one of the tasks. Linguistics literature describes the relationship between task complexity and outcome measures such as linguistic complexity (e.g., advanced language structures), accuracy (e.g., avoiding errors), and fluency (e.g., speech produced without interruption at a normal speaking rate).

The trade-off hypothesis describes how task complexity and task characteristics differentially affect complexity, accuracy, and fluency (CAF) outcomes. Often, the speaker's attention is focused on one or two of the outcomes at the expense of the others (Skehan, 1998). For example, narrative tasks typically yield high levels of complexity and lower levels of accuracy and fluency. Reporting of information that is concrete and familiar tends to beget relatively accurate and fluent reports. Furthermore, tasks that 
require manipulation of information encourage speakers to focus on creating complex speech, which leaves few attentional resources to monitor the accuracy and fluency of that speech (Skehan, 2009). Linguistic tasks with clear chronological structures tend to elicit accurate and fluent reports (Skehan, 2001).

Relevance to the $\mathbf{C I}$. The change perspective mnemonic requires witnesses to consider aspects of the narrative that may be less familiar (e.g., figuring out what details a bystander would have seen). The reverse order mnemonic disrupts witnesses' clear chronological order. The CI mnemonics are linguistically complex tasks, which may decrease the accuracy and fluency of witness reporting, especially for NNESs. Witness accuracy is critical for solving crimes; therefore, maintaining accuracy and maximizing productivity within a CI presents a challenge for interviewers and NNESs.

\section{The CI: Linguistic/Cognitive Complexity and Social Support}

As mentioned earlier, some of the CI mnemonics-like the reverse order or change perspective techniques - may be cognitively demanding. Since the witness must recall and manipulate information, these techniques require more cognitive resources than simple free recall instructions. Imposing a load upon cognitive resources (cognitive load) has been successfully used in deception detection literature to overload liars (Vrij, Leal, Mann, \& Fisher, 2012). Lying — much like speaking a non-native language — is a cognitively demanding task; liars must concurrently suppress the truth, create a lie, monitor their own behaviors, monitor the interviewer's behaviors, and more. If liars are cognitively overloaded, they will not be able to maintain their lies, thus eliciting more cues to deception. Vrij and colleagues (2012) asked liars and truth-tellers to recount a route home in chronological order or reverse order. Although lying participants provided 
fewer details in the lying condition compared to the truthful condition regardless of report order, the effect size within the reverse order condition was quite large $(d=.72)$ compared to the effect size for the chronological order condition $(d=.38)$. The large effect size suggests that there is an effect of the reverse order technique on participants' abilities to perform cognitively demanding tasks like lying.

Another study by Ewens, Vrij, Mann, and Leal (2016c) tested the reverse order technique specifically with NNESs. Their participants were instructed to either lie or tell the truth in English or in their native languages with the assistance of an interpreter. Most pertinent to the present study is the number of details reported by participants telling the truth. The number of details given in chronological order was comparable between NNESs speaking English and those speaking their native languages. However, when interviewed with the reverse order mnemonic, NNESs speaking English gave somewhat fewer details than those speaking through an interpreter. The difference between native and non-native speakers may suggest that the reverse order mnemonic works as intended when witnesses can use their native language, but that NNESs are at a slight disadvantage when speaking a non-native language.

Despite these potential shortcomings, there are components of the CI that may be especially helpful with NNESs. As discussed earlier, witnesses are frequently hesitant to communicate with interviewers for many reasons. Non-native English speakers are under an additional layer of social pressure when asked to create speech in a non-native language. In an eyewitness interview, NNESs may feel the additional burden of not being able to communicate effectively on top of the discomfort of describing personal, stressful events to the interviewer. The social variables described by MacIntyre and colleagues 
(1998) suggest that interviewers can manipulate the social context to help NNES witnesses overcome barriers to reporting. Specifically, instructing witnesses to report everything they can remember and transferring control to the witness (e.g., by telling the witness that $\mathrm{s} / \mathrm{he}$ is the expert in the interview) may help witnesses feel more comfortable speaking in a second language during the unfamiliar context of a police interview. These instructions may help to increase the witness's self-confidence in the moment (by taking

on the role of the expert). Furthermore, if the witness knows that seemingly unimportant, out of sequence, or corrected details are acceptable (after receiving the "report everything" instruction), s/he may feel less insecure about speaking a non-native language. So, these introductory instructions recommended by the CI may be especially beneficial for NNES witnesses.

\section{The Present Study}

Given the cognitive complexity of speaking in a second language, it is possible that the cognitively demanding CI mnemonics are not effective for NNES witnesses. The present study will test two of the CI mnemonics within native English-speaking and NNES samples. The primary aim of the current study is to test whether these two CI mnemonics are effective interviewing techniques for NNES witnesses. The study will contain three conditions, which test two of the CI mnemonics in native and NNES samples. The two mnemonics tested in the current dissertation are (1) the reverse order instruction and (2) the transfer of control instruction. The reverse order mnemonic was chosen because it is cognitively demanding and linguistically complex, yet it typically "works" with native speakers. 
Regarding CI mnemonics, the current study addressed two research questions. First, does the reverse order mnemonic elicit more details than a control, "try again" prompt? Second, does inclusion of the transfer of control instruction elicit more details compared to control interview instructions?

Further, the current study tested CI mnemonics among native English speakers and NNESs. Regarding language proficiency, the current study addressed two research questions: First, do NNESs report fewer details in response to the reverse order mnemonic than the control, "try again" prompt? Second, do NNESs report more details when given the transfer of control instruction than the control interview instructions?

Finally, the current study addressed the interactive effects of language and interview instructions. Specifically, the current study addressed two main questions regarding interactive effects: First, is the reverse order prompt more helpful for native speakers than NNESs? Second, is the transfer of control instruction particularly helpful for NNESs as well as native speakers?

Hypotheses. In general, main effects of interview condition and English language proficiency are expected. Participants who receive CI mnemonics/instructions are expected to recall significantly more details than those who do not receive CI mnemonics/instructions (i.e., the control group). Native English speakers are expected to provide more details than NNESs. Furthermore, an interaction between English proficiency and interview condition is expected such that native English speakers will benefit from the reverse order mnemonics relative to a control interview whereas NNESs will not benefit from those mnemonics relative to a control interview. However, both 
native speakers and NNESs are expected to benefit from the transfer of control condition relative to a control interview.

Furthermore, the current study will provide evidence of the general effectiveness of two CI mnemonics in isolation. It will be possible to compare each of the CI mnemonics to the control condition. Although some previous research has addressed the role of individual CI mnemonics (testing the reverse order, change perspective, and “report all” mnemonics), it was likely underpowered (Milne \& Bull, 2002). Thus, a secondary aim of the current study is to test whether each of the selected CI mnemonics provides an added benefit to recall absent the rest of the CI. Research on the CI typically compares full CIs to standard or control interviews, and relatively little research has investigated the contribution of each individual mnemonic (Memon \& Higham, 1999). 


\section{METHOD}

\section{Participants}

Ninety-eight (98) participants were recruited. All participants were required to be at least 18 years of age. Sixty-four (65.3\% of the total sample) native English speakers were recruited from the Psychology Department online participant pool (i.e., Sona Systems) at Florida International University in exchange for course research credit. Thirty-four (34.7\% of the total sample) non-native English speakers were recruited; these participants were recruited via Florida International University's English Language Institute (ELI), Sona Systems, and from the community via flyers. The ELI is part of Florida International University's Office of Faculty and Global Affairs. It provides intensive English language courses to international students. Students enrolled in the ELI are assigned a course level on the basis of standardized test scores; level 1 represents the lowest level of English proficiency, and level 6 represents the highest level of proficiency served by the ELI. After level 6, students are considered ready to attend classes taught in English at the university level. Participants from the ELI were recruited for the current study via in-person announcements in their classes and computer labs. The study was framed as an opportunity to practice English communication outside of the ELI classes. Participants were recruited from ELI classes serving levels 2 through 5. Because participants in level 1 would not be able to respond in English whatsoever, these participants were not recruited; there were no participants in the ELI's level 6 courses during recruitment. Participants who indicated interest during course announcements were scheduled to participate via email. Recruitment flyers were posted in the ELI to 
attract participants. ELI participants who volunteered after January 2019 were compensated with a $\$ 10$ Starbucks gift card.

Community participants were recruited by posting flyers around Florida International University campus and in local, off-campus coffee shops and restaurants. Participants could call, text message, or email a dedicated study email or Google Voice number to schedule their participation. Other community members were recruited by word of mouth; for example, some participants referred their friends and family members to the study. Community participants were compensated with a $\$ 10$ Starbucks gift card.

The mean age of participants was $23.34(S D=6.29)$. Sixty participants $(61.2 \%)$ identified as female, $35(35.7 \%)$ identified as male, and 3 did not respond. In response to highest level of education enrolled/completed, 10 participants (10.2\%) reported high school, $75(76.5 \%)$ reported college (BA/BS), 7 (7.1\%) reported graduate school (MA/MS), and $1(1.0 \%)$ reported doctoral level (PhD/JD/MD); 5 participants $(5.1 \%)$ did not respond.

Native English speakers. Among native English speakers, the mean age of participants was $22.70(S D=5.79)$. Thirty-eight $(59.4 \%)$ participants identified as female, $25(39.1 \%)$ identified as male, and 1 (1.6\%) did not respond. In response to highest level of education completed/enrolled, 7 participants $(10.9 \%)$ reported high school, $54(84.4 \%)$ reported college $(\mathrm{BA} / \mathrm{BS})$, and 1 (1.6\%) reported graduate school (MA/MS); 2 participants $(3.1 \%)$ did not respond.

Non-native English speakers. Among NNES, the mean age of participants was $24.58(S D=7.09)$. Twenty-two $(64.7 \%)$ participants identified as female, $10(29.4 \%)$ identified as male, and $2(5.9 \%)$ did not respond. In response to highest level of education 
completed/enrolled, 3 participants (8.8\%) reported high school, 21 (61.8\%) reported college (BA/BS), $6(17.6 \%)$ reported graduate school (MA/MS), and 1 (2.9\%) reported graduate school (PhD/JD/MD); 2 participants (3.1\%) did not respond. Over half (55.9\%, $N=19$ ) of NNESs reported Spanish as their first language; see Table 1 for a full breakdown of NNESs' first languages.

Table 1. First languages reported by NNES participants

\begin{tabular}{lll}
\hline Language & $N$ & Percent of \\
& & NNES sample \\
\hline Spanish & 19 & $55.9 \%$ \\
Russian & 5 & $14.7 \%$ \\
Portuguese & 3 & $8.8 \%$ \\
Arabic & 1 & $2.9 \%$ \\
Chinese & 1 & $2.9 \%$ \\
French & 1 & $2.9 \%$ \\
Italian & 1 & $2.9 \%$ \\
Japanese & 1 & $2.9 \%$ \\
Papiamentu & 1 & $2.9 \%$ \\
Ukrainian & 1 & $2.9 \%$ \\
\hline Total & 34 & $100 \%$ \\
\hline & & \\
\hline
\end{tabular}

Language condition categorization. Participants who volunteered from the psychology participant pool or from the community were considered non-native English 
speakers if they reported their ability to speak English as less than "very well" on the language history questionnaire. That is, on a scale from 1 [very poor] to 7 [native-like], participants who reported that their English speaking ability was less than 6 [very well] (i.e., 5 [well] or below) were categorized as non-native English speakers. See question 11 in Appendix A. All participants recruited from the ELI were considered non-native English speakers. All other participants were categorized as native speakers. Any participants with incomplete language data (e.g., questions regarding language ability were skipped not filled out for English) were grouped with native speakers if they signed up through Sona Systems or with NNESs if they were recruited from the ELI.

Following categorization, participants' average self-rating of English speaking ability was $6.74(S D=0.50)$ among native English speakers and $5.24(S D=1.46)$ among NNESs. An independent samples $t$-test indicated that the difference was statistically significant, $t(85)=6.78, p<.001$, Cohen's $d=1.35$. For further details on participants' ratings of listening, reading, and writing abilities, see Table 2.

Table 2. Participant self-ratings of English ability (Language History Questionnaire, question 11)

\begin{tabular}{lllll}
\hline & \multicolumn{2}{c}{ Native } & \multicolumn{2}{c}{ NNES } \\
\cline { 2 - 5 } English ability & $M$ & $S D$ & $M$ & $S D$ \\
\hline Listening ability & 6.83 & 0.42 & 5.73 & 1.26 \\
Speaking ability & 6.74 & 0.50 & 5.24 & 1.46 \\
Reading ability & 6.81 & 0.52 & 5.85 & 1.06 \\
Writing ability & 6.59 & 0.83 & 5.39 & 1.27 \\
\hline
\end{tabular}




\section{Design}

The study conformed to a 3 (interview technique: control interview, transfer of control, reverse order $) \times 2$ (English proficiency: native, non-native) between-participants design, with participants randomly assigned to the interview technique condition via block randomization.

\section{Materials}

Language History Questionnaire. Each participant completed a language history questionnaire (LHQ; Li, Sepanski, \& Zhao, 2006; Li, Zhang, Yu, \& Zhao, 2019). The LHQ included questions about the respondent's native language, context of first and second language use (e.g., at work, in the classroom, in casual conversations), and cultural identity. The LHQ included demographic questions including age, sex, education level, country of origin, and country of residence. It also included self-report questions assessing context and self-rated proficiency of all languages spoken by the participant. For example, one question asked the age at which participants starting using each of their languages at home, at school, and at work; another question asked participants to rate their ability to listen, speak, read, and write each of their languages. Other questions asked participants to estimate the number of hours per day they spend consuming media in different languages (e.g., television, radio, reading/writing for school or work) and the number of hours per day they spend speaking with family members, friends, classmates, and coworkers in different languages. For the full LHQ, see Appendix A.

Video stimulus. The to-be-remembered event was a video of a mock theft. The video was 2 minutes, 10 seconds long. The video begins with the cameraperson traveling 
through the DM building of Florida International University. The cameraperson enters the psychology wing of the building and finds two perpetrators in an office space. A male and female perpetrator take several items, including a laptop, some books, and a backpack from the office space. They leave the office space and exit the psychology hallway. The video included minimal language content (e.g., the word "Psychology" appears on the hallway, there is a sign on the office door) and no spoken language. See Figure 13 and Figure 14.

Informed consent. Informed consent was provided in English, Spanish, or Portuguese, depending on participant preference. Participants who were native speakers of other languages gave informed consent in English; the research assistant offered to reschedule the session to obtain translated consent documents, but participants indicated a preference for English. The option was always given before the participant gave informed consent to avoid coercion.

Back translation. Informed consent documents were translated from English into Spanish and Portuguese by bilingual speakers of Spanish and Portuguese. The translated (Spanish and Portuguese) documents were then translated back into English by independent bilingual Spanish and Portuguese speakers.

\section{Research Assistant Training}

Five psychology undergraduate students were recruited to serve as research assistants (i.e., running study sessions, including interviews, with participants) for the current study. All five research assistants participated in the lab for internship credit. Each research assistant was interviewed individually by the author prior to joining to research team. The preliminary interview was used to assess the research assistant's 
academic background (including courses taken, especially legal psychology), reliability, and communication skills (including additional languages spoken). All five research assistants had taken a course in legal psychology. All five research assistants were bilingual in Spanish.

All research assistants were thoroughly trained in the procedure before interacting with participants. First, the protocol and all possible interview conditions were explained to each research assistant. Each research assistant was responsible for knowing and administering all three possible experimental interview conditions. Second, each research assistant engaged in at least one practice session either with the author or in pairs with each other. During the practice sessions research assistants were tested on potential participant questions and points of confusion (e.g., misunderstanding the experimental interview instruction or misunderstanding LHQ questions). The author provided feedback to each research assistant following his/her practice session, and scheduled additional practice sessions if necessary. Finally, research assistants were scheduled with a participant from the undergraduate pool for their first real interview session; each research assistant's first real interview session was supervised by the author.

\section{Procedure}

See Figure 5 for an outline of the study. See Appendix B for the full native English condition script and Appendix C for the full NNES condition script. The same bilingual research assistant administered the entire experiment. Whenever possible, the research assistant was bilingual in English and the participant's native language. Participants arrived at the lab and provided informed consent in English or their native language, according to the participant's preference. After obtaining informed consent and 
clarifying any participant questions, which may have occurred in Spanish or Portuguese, the entire remainder of the study occurred in English. To ensure enough participants in each cell, the interview conditions were randomized in blocks. A list of conditions was generated with equal numbers of each condition; the complete list with all necessary conditions was then randomized. Block randomization ensured that each condition received equal numbers of participants. A separate list was randomized for each of the two language conditions. 


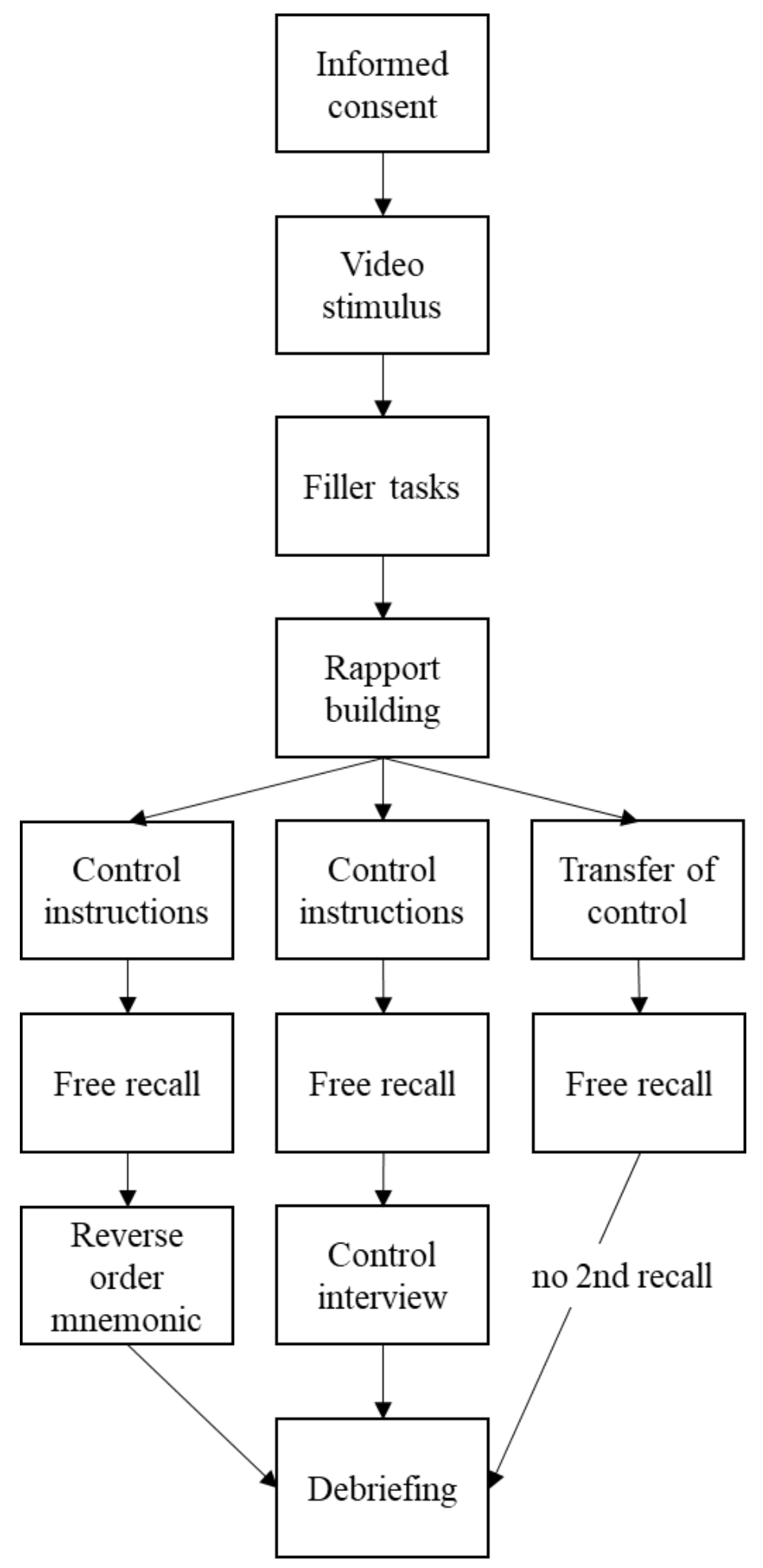

Figure 5. Study protocol. 
Participants were told that they would watch a video, and they were asked to pay attention to the video because the research assistant would ask questions about it later. Participants then viewed the mock theft video. The video contained several sequences of action, but did not contain any dialogue. Following the video, participants completed the LHQ (see Appendix A) for approximately 20 minutes.

After completing the LHQ, the research assistant began the interview according to the randomly assigned interview condition. All interviews were conducted in English and were audio recorded in their entirety. All interviews began with a short rapport-building phase. To establish rapport, the research assistant asked questions to the participant (e.g., "You're graduating this semester? Did you do the GRE yet?") and responded briefly to draw a connection with the participant (e.g., “Oh, I didn't know that programs in Europe don't require the GRE!"). Each experimental condition then diverged according to the assigned protocol.

Control interview condition. For the full control condition protocol, see Appendix D. The control interview began with a control set of instructions. The participant was informed that s/he just saw a video of a crime being committed and that the research assistant would ask some questions about that. Then, participants were asked a free recall question ("Please tell me everything you can remember about what happened"). The research assistant was trained to not interrupt the participant at any point. After the participant finished recalling, the research assistant then asked the participant to engage in a second free recall ("Please tell me, again, everything that you remember about what happened"). Participants were asked to verbally recount the instruction to confirm their understanding. Research assistants were trained to re-explain 
the instruction if participants incorrectly described the instruction or indicated misunderstanding. At the end of the second narrative, the research assistant confirmed that the participant had finished recalling ("Is there anything else you can tell me about what happened?"), and then concluded the interview.

Reverse order interview condition. For the full reverse order condition protocol, see Appendix E. The reverse order interview began with the control set of instructions. The participant was informed that s/he just saw a video of a crime being committed and that the research assistant would ask some questions about that. Then, participants were asked a free recall question ("Please tell me everything you can remember about what you saw"). The research assistant was trained to not interrupt the participant at any point. After the participant finished recalling, the research assistant asked the participant to recall the crime in reverse order, starting with the end of the crime and working backward to the beginning. Participants were asked to verbally recount the instruction to confirm their understanding. Research assistants were trained to re-explain the instruction if participants incorrectly described the instruction or indicated misunderstanding. At the end of the second narrative, the research assistant confirmed that the participant was finished recalling ("Is there anything else you can tell me about what happened?"), and then concluded the interview.

Transfer of control interview condition. For the full transfer of control condition protocol, see Appendix F. The CI instruction interview began with the control set of instructions. The participant was informed that s/he just saw a video of a crime being committed and that the research assistant would ask some questions about that. After the control instructions, the research assistant gave instructions transferring control 
to the participant ("You saw the crime, so you know what happened. I don't know what happened, so I need you to tell me. I'm just going to take notes while you talk.”). Then, participants were asked a free recall question ("Please tell me everything you can remember about what happened"). The research assistant was trained to not interrupt the participant at any point. After the participant finished recalling, the research assistant confirmed that the participant was finished recalling ("Is there anything else you can tell me about what happened?"), and then concluded the interview. There was no second recall phase in the transfer of control condition.

\section{Closing the Session}

Following the assigned interview condition, the research assistant debriefed and dismissed the participant. Specifically, the research assistant told the participant that “[d]ifferent participants receive different instructions and different interview questions.

We are testing whether native and non-native English speakers respond differently when interviewed with these different techniques." (See Appendices A and B for the full script.) Participants recruited from the psychology participant pool were compensated with extra credit in their psychology course via Sona Systems, the online psychology participant pool. Participants recruited from the ELI and from the community after January 2019 were compensated with a \$10 Starbucks gift card; participants recruited from the ELI before January 2019 volunteered to participate for no compensation.

\section{Transcription and Scoring}

The recorded audio of each interview was transcribed and then coded for two constructs: units of information and detail accuracy. The two constructs are consistent with previous research in the field (e.g., Evans \& Fisher, 2011), and they represent 
information that would be useful to police investigators. First, units of information were operationalized as the smallest statements made by participants that contained verifiable information. . A unit of information was defined as a noun, adjective, active verb (i.e., not "was" or "had"), adverb, or preposition. One research assistant was trained on the unitization protocol and then unitized all of the transcripts. To illustrate the coding scheme, the statement "she was holding it [a bag] by the side as if it was heavy" contains four units: (1) she, (2) was holding it, (3) by the side, (4) as if it was heavy." See Appendix $\mathrm{H}$ for the unitization protocol for the current study.

The unitized transcripts were subsequently scored for accuracy. See Appendix I for the scoring protocol for the current study. A list of critical details (i.e., a master list) was compiled from the video by two independent research assistants (see Appendix J). The critical detail list and the video itself were provided to the scorers. The total number of unique, verifiable details reported in each interview was counted. Scorers first determined whether the unit was new or repeated. Repeated details were not scored further for accuracy. New (non-repeated) units were scored as accurate if they appeared on the master list or were verified by checking the video. Details were scored as inaccurate if they contradicted the details in the master list and/or contradicted a detail in the video. Details were scored as subjective if they reflected an opinion or assumption made by the participant. Details were considered "not scoreable" if they did not the fit the aforementioned categories; that is, non-scoreable details referred to the video but could not be verified for accuracy and were not considered subjective statements. Using the example statement provided above ("she /was holding it [a bag] /by the side, /as if it was heavy"), detail 1 is accurate (there was a female thief), details 2 and 3 are inaccurate (she 
was not holding the bag, and what she was holding [books] was not by its side), and detail 4 is subjective ("heavy" is an opinion).

Two research assistants were trained on the scoring protocol. Each research assistant met with the author individually to learn the scoring rules. After discussing the rules and any questions, the research assistant scored approximately three transcripts with direct supervision of the author. After training, each research assistant scored $10 \%$ of the transcripts to obtain inter-rater reliability (see the following section).

\section{Inter-rater Reliability}

Two independent research assistants were trained to score unitized transcripts. Both scorers scored $10 \%$ of the transcripts to assess inter-rater reliability. Intraclass correlation coefficients (ICCs) for consistency between the two scorers were computed in SPSS using two-way mixed models. Reliability analyses revealed acceptable levels of consistency between scorers across all scores. In general, ICCs above .90 indicate excellent reliability, ICCs between .75 and .89 indicate good reliability, and ICCs between .5 and .74 indicate moderate reliability (Cicchetti, 1994; Koo \& Li, 2016). In the present study, excellent reliability was achieved for accurate and inaccurate details. Reliability for subjective details was good, and reliability for repeated details was moderate to good. Intraclass correlations ranged from .52 to .98; see Table 3. After achieving reliability, each scorer was assigned to independently score a portion of the remaining transcripts. One of the scorers was designated as the primary scorer; for the $10 \%$ of transcripts double scored, the primary scorer's scores was used for data analysis. 
Table 3. Intraclass correlation coefficients for detail scores.

\begin{tabular}{lll}
\hline Detail type & ICC & Quality \\
\hline Accurate & .97 & Excellent \\
Inaccurate & .90 & Excellent \\
Subjective & .75 & Good \\
Not scoreable & .52 & Moderate \\
Repeated & .73 & Moderate to good \\
\hline
\end{tabular}




\section{RESULTS}

\section{Manipulation Checks}

To confirm that the reverse order technique was indeed cognitively demanding for participants, a one-way ANOVA was used to assess differences in participants' ratings of difficulty between interview conditions. Participants were asked how hard it was to complete the experimental interview condition in their assigned condition (i.e., "how hard was it to report your memory in reverse order?" or "how hard was it to report your memory a second time?" or "how hard was it to report everything you could remember?" on a scale where $1=$ not hard at all and $10=$ very hard; see Appendix A). There was a significant effect of interview condition on difficulty ratings, $F(2,89)=9.68, p<.001$, partial $\eta^{2}=.18$. Pairwise comparisons indicated that participants in the reverse order condition reported that the interview was harder $(M=6.30, S D=2.37)$ than participants in the control $(M=3.41, S D=2.12)$ and transfer of control $(M=4.42, S D=2.93)$ conditions; there was no significant difference in participants' difficulty ratings of the control and transfer of control interviews. The significant difference in difficulty ratings between interview groups suggests that the reverse order technique is indeed more cognitively challenging than the other techniques.

Further, an independent samples $t$-test was conducted on participants' responses to the question, "How comfortable were you reporting your memory in English?" (wherein $1=$ very uncomfortable and $10=$ very comfortable). Results indicated that participants in the NNES group reported feeling significantly less comfortable $(M=6.55$, $S D=2.38)$ than native English speakers $(M=9.19, S D=1.99), t(46.58)=5.19, p<.001$. Note that Levene's test for equality of variances was violated for the current test, $F=$ 
$4.04, p=.048$; thus, adjusted degrees of freedom are reported. The significant difference in comfort ratings between language groups suggests that participants did differ in English proficiency between the two language conditions, as intended.

\section{Units of Information}

All analyses in the following section are performed with units of information as the dependent variable. The units of information variables contain all the units reported by the participant, including units that are inaccurate, unscoreable, and repeated. Means and standard deviations are reported in text for significant comparisons; descriptive statistics for non-significant comparisons are reported in tables.

Reverse order condition. A $2 \times 2$ ANOVA, including interview condition (control, reverse order) and proficiency level (native, NNES) as independent variables, was used to detect mean differences between these groups on the outcome of total units of information provided in the full interview (i.e., across both recall attempts, including repeated details). There was a significant main effect of language, $F(1,57)=4.09, p=$ .048 , partial $\eta^{2}=.07$, with native English speakers $(M=80.63, S D=40.42)$ reporting more details than NNES $(M=60.76, S D=28.06)$. See Figure 6 for estimated marginal means and standard errors of total detail quantity across interview conditions and language groups. There was no significant effect of interview condition on total units of information provided across the two interview conditions, $F(1,57)=2.55, p=.116$, partial $\eta^{2}=.04$. However, there was a marginally significant interaction, $F(1,57)=3.12$, $p=.083$, partial $\eta^{2}=.05$. Specifically, native English speakers provided more details overall in the control condition than the reverse order condition; the difference was not 
significant among NNESs. See Table 4 for estimated marginal means and standard deviations for comparisons between the control and reverse order conditions.

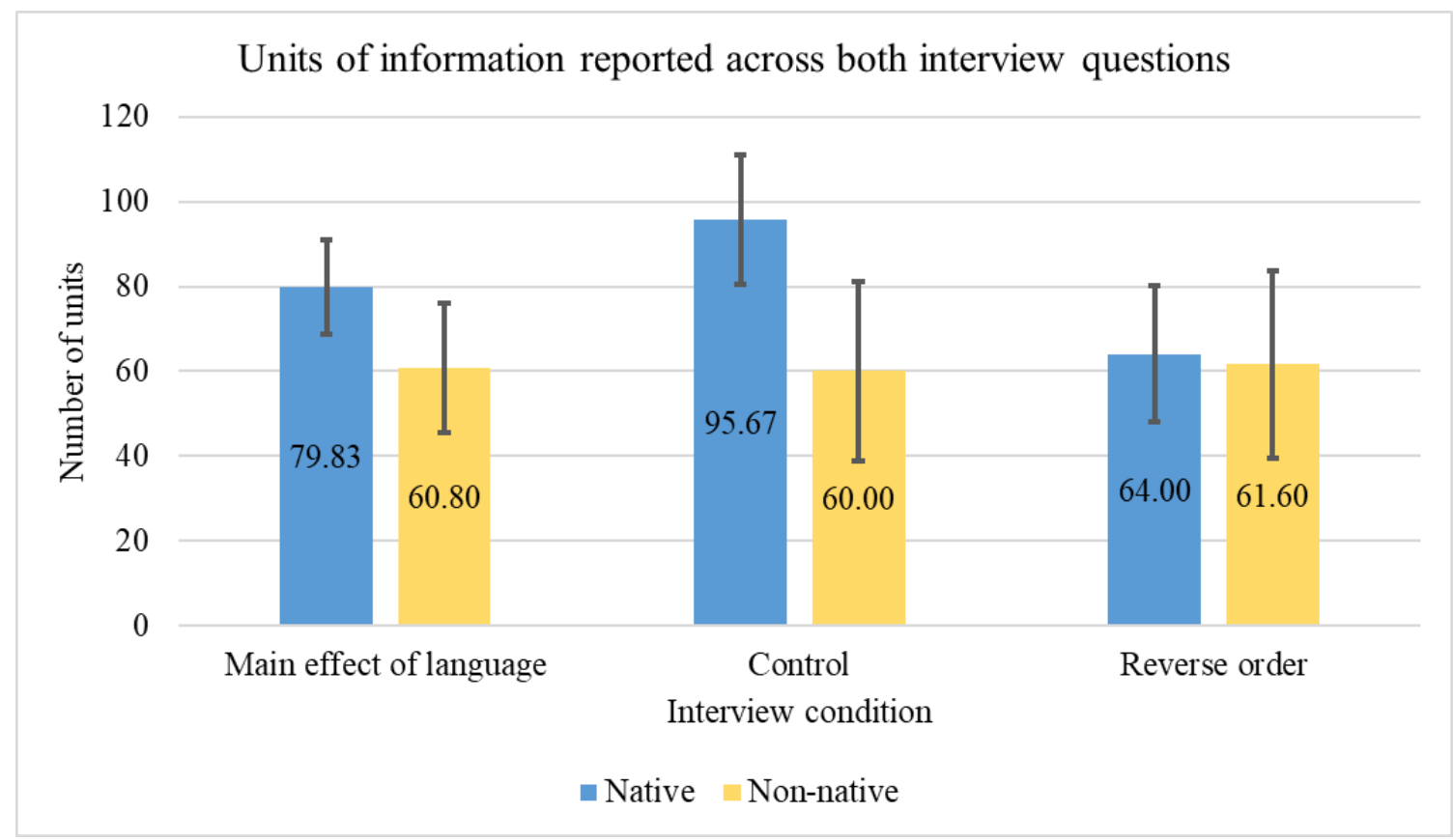

Figure 6. Estimated marginal means and $95 \%$ confidence intervals for units of information reported across both interview questions (control vs. reverse order conditions).

Table 4. Estimated marginal means and standard deviations for reverse order analyses.

\begin{tabular}{|c|c|c|c|c|c|c|c|}
\hline \multirow{3}{*}{\multicolumn{2}{|c|}{ Native }} & \multicolumn{2}{|c|}{ Question 1} & \multicolumn{2}{|c|}{$\begin{array}{l}\text { Question } 2 \text { (i.e., } \\
\text { Experimental) }\end{array}$} & \multicolumn{2}{|c|}{ Total Details } \\
\hline & & $M$ & $S D$ & $M$ & $S D$ & $M$ & $S D$ \\
\hline & & & & & & & \\
\hline & Control & 40.95 & 18.55 & 54.71 & 29.76 & 95.67 & 45.60 \\
\hline & Reverse order & 35.58 & 17.92 & 28.42 & 13.34 & 61.60 & 25.94 \\
\hline & Total & 38.40 & 18.23 & 42.23 & 26.70 & 80.63 & 40.41 \\
\hline
\end{tabular}


NNES

$\begin{array}{lrrrrrr}\text { Control } & 29.81 & 15.75 & 30.18 & 16.09 & 60.00 & 29.37 \\ \text { Reverse order } & 34.20 & 17.86 & 27.40 & 13.67 & 61.60 & 28.11 \\ \text { Total } & 31.90 & 16.51 & 28.86 & 14.68 & 60.76 & 28.06\end{array}$

Total

$\begin{array}{lrrrrrr}\text { Control } & 37.13 & 18.20 & 46.28 & 29.76 & 83.41 & 43.77 \\ \text { Reverse order } & 35.10 & 17.59 & 28.07 & 13.22 & 63.17 & 26.23\end{array}$

First recall attempt only. A $2 \times 2$ ANOVA, including interview condition (control, reverse order) and proficiency level (native, NNES) as independent variables, was used to detect mean differences between these groups on the outcome of units of information provided in the first recall attempt of each interview condition. There was no significant main effect of language, $F(1,57)=1.70, p=.197$, partial $\eta^{2}=.03$. There was also no significant main effect of interview condition on total units of information in the first recall attempt, $F(1,57)=0.01, p=.918$, partial $\eta^{2}<.01$. The lack of differences is unsurprising, considering the initial questions were exactly the same in both conditions. There was also no significant interaction, $F(1,52)=1.03, p=.314$, partial $\eta^{2}=.02$. See Figure 7 for estimated marginal means and standard errors of detail quantity in response to the first free recall question across interview condition and language group. 


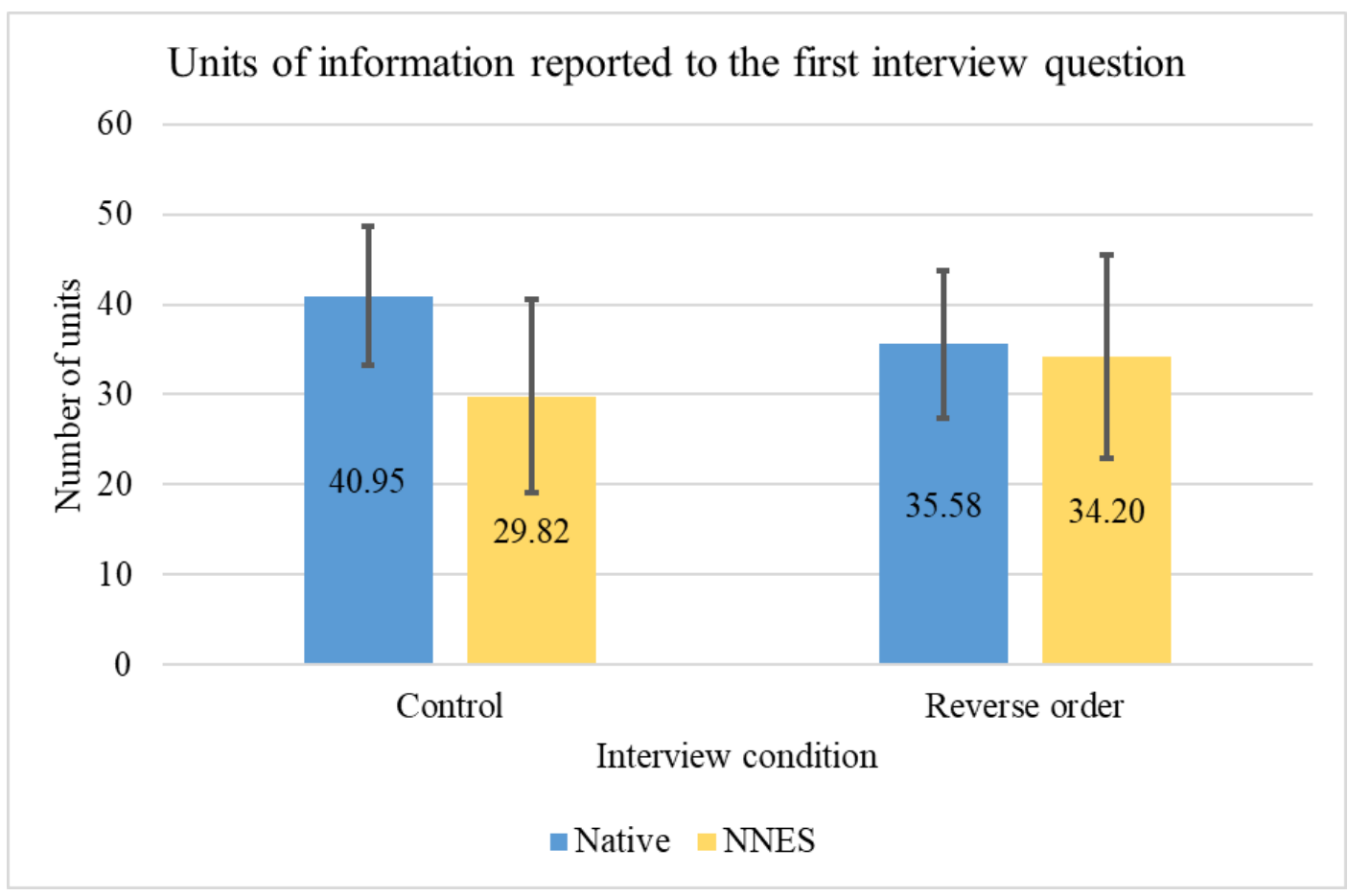

Figure 7. Estimated marginal means and $95 \%$ confidence intervals for units of information reported during the first interview question (control vs. reverse order conditions).

Experimental interview question only. A $2 \times 2$ ANOVA, including interview condition (control, reverse order) and proficiency level (native, NNES) as independent variables, was used to detect mean differences between these groups on the outcome of units of information provided in response to the experimental interview question (i.e., the second-recall or reverse-order question). There was a significant main effect of language, $F(1,57)=5.08, p=.028$, partial $\eta^{2}=.08$. Native English speakers $(M=42.23, S D=$ 26.70) reported significantly more details than NNES $(M=28.56, S D=14.68)$. There was also a significant main effect of interview condition on units of information provided, $F(1,57)=6.57, p=.013$, partial $\eta^{2}=.10$. Participants in the control condition $(M=46.28, S D=28.20)$ reported significantly more details than participants in the 
reverse order condition $(M=28.07, S D=13.22)$. These main effects were qualified by a significant interaction between language and interview condition on units of information, $F(1,57)=4.30, p=.043$, partial $\eta^{2}=.07$. Pairwise comparisons revealed that native English speakers reported more units in response to the second interview question in the control condition $(M=54.71, S D=29.76)$ compared to the reverse order condition $(M=$ 28.42, $S D=13.34), p<.001$. However, the difference between interview conditions was not significant for NNES participants $\left(M_{\text {control }}=30.18, S D_{\text {control }}=16.09 ; M_{\text {reverse }}=27.40\right.$, $\left.S D_{\text {reverse }}=13.67\right)$. See Figure 8 and Table 5 for estimated marginal means and standard deviations of quantity of details reported to the experimental (second) recall question across interview condition and language group. 


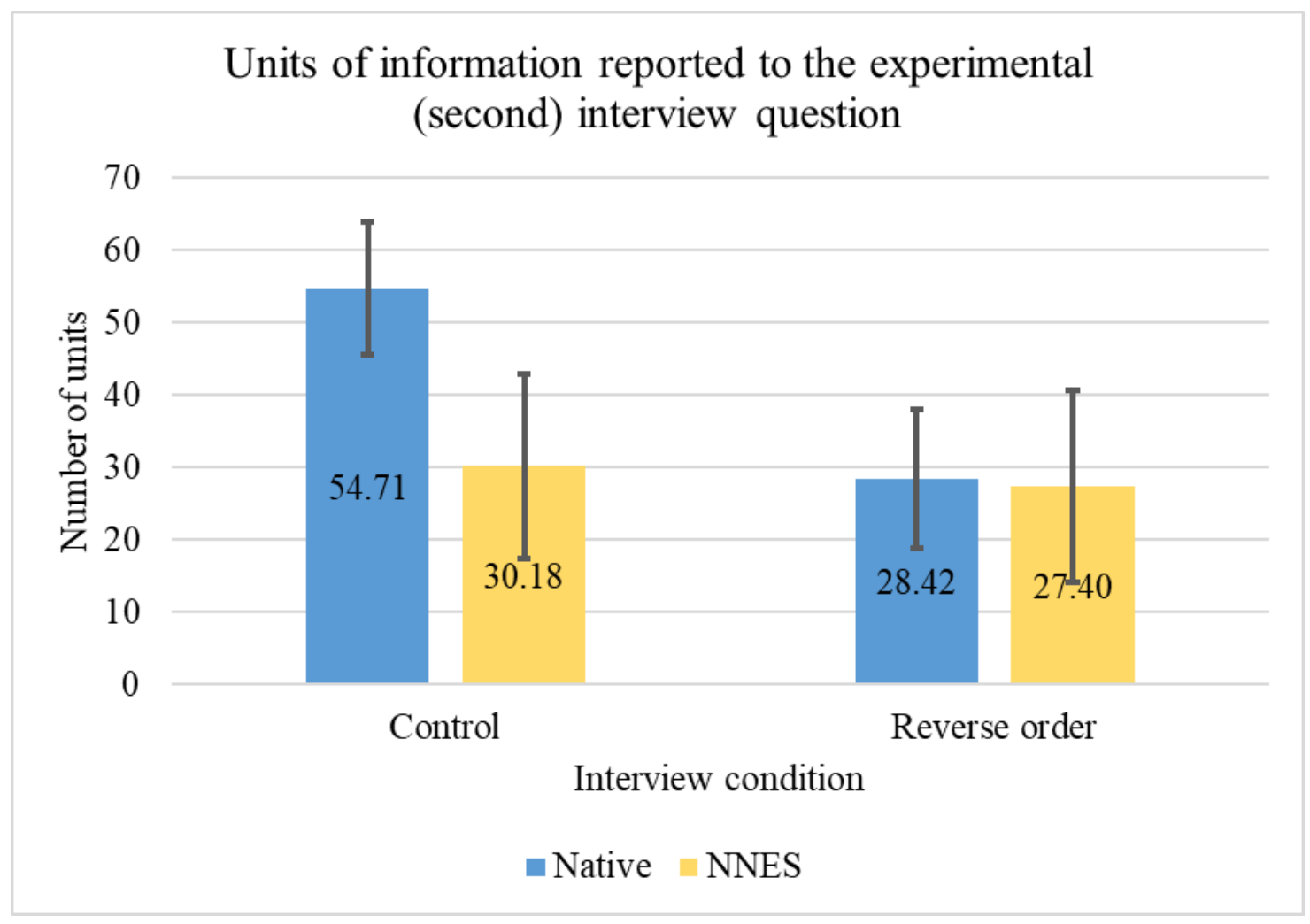

Figure 8. Estimated marginal means and $95 \%$ confidence intervals for units of information reported to the experimental interview question (control vs. reverse order conditions)

Transfer of control condition. A $2 \times 2$ ANOVA, including interview condition (control, transfer of control) and proficiency level (native, NNES) as independent variables, was used to detect mean differences between these groups on the outcome of units of information provided for the full interview in the transfer of control condition (which only included one recall attempt) and for the first recall attempt only in the control interview condition. There was a significant main effect of language on units of information provided, $F(1,64)=6.12, p=.016$, partial $\eta^{2}=.09$. Native English speakers $(M=43.20, S D=21.14)$ provided significantly more details than non-native English speakers $(M=31.21, S D=13.70)$. See Figure 9. There was no significant main effect of 
interview condition on units of information provided, $F(1,64)=.502, p=.481$, partial $\eta^{2}$ $=.01$. There was also no significant interaction effect, $F(1,64)=.03, p=.858$, partial $\eta^{2}$ $<.01$.

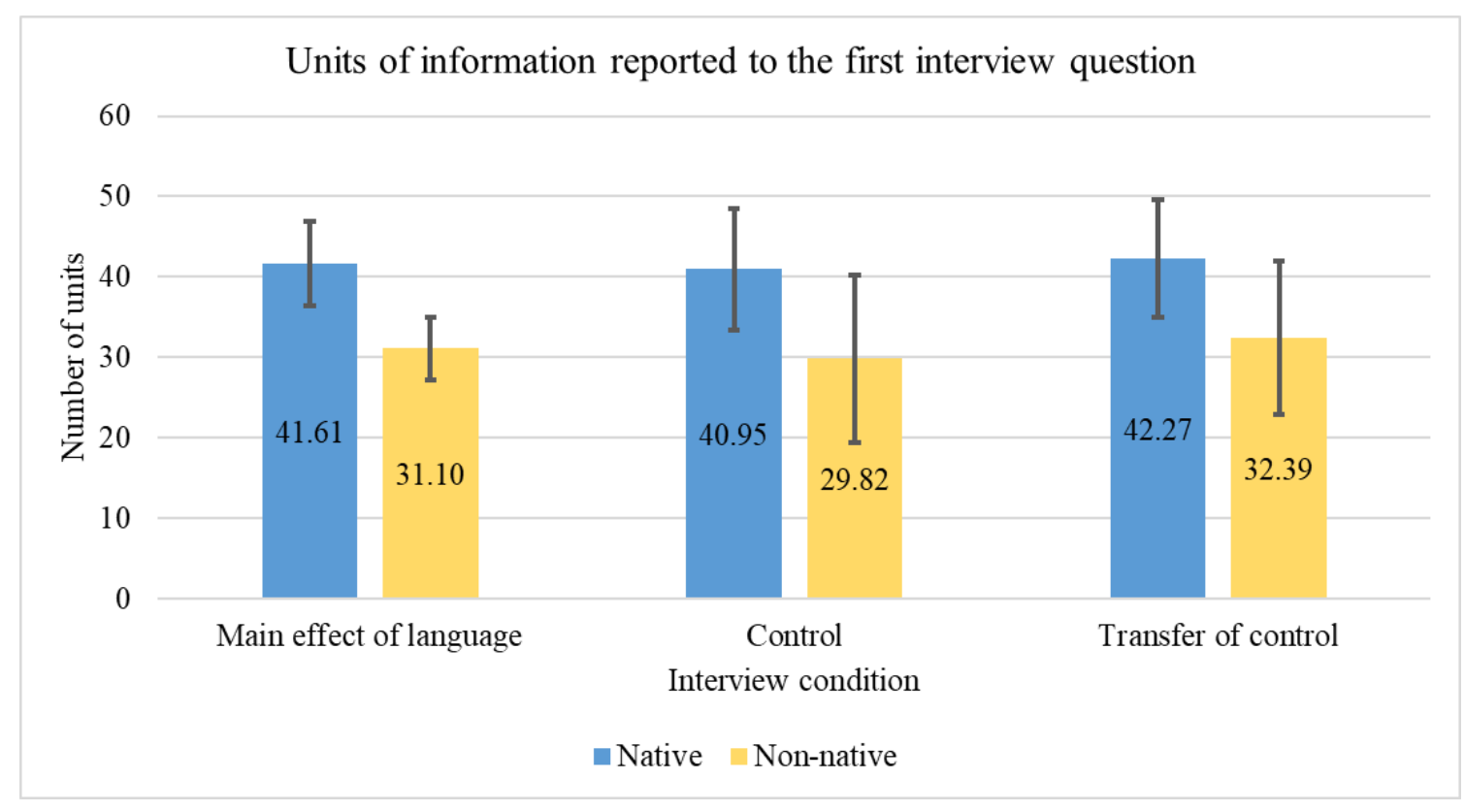

Figure 9. Estimated marginal means and $95 \%$ confidence intervals for units of information reported in the first interview question (control vs. transfer of control conditions). 
Table 5. Estimated marginal means and SDs for transfer of control condition

\begin{tabular}{|c|c|c|c|}
\hline & & \multicolumn{2}{|c|}{ Question 1} \\
\hline & & M & SD \\
\hline \multicolumn{4}{|l|}{ Native } \\
\hline & Control & 40.95 & 18.55 \\
\hline & Transfer of & 45.26 & 23.47 \\
\hline & control & & \\
\hline & Total & 43.20 & 21.14 \\
\hline \multicolumn{4}{|l|}{ NNES } \\
\hline & Control & 29.82 & 15.75 \\
\hline & Transfer of & 32.38 & 12.24 \\
\hline & control & & \\
\hline & Total & 31.21 & 13.70 \\
\hline \multicolumn{4}{|l|}{ Total } \\
\hline & Control & 37.13 & 18.20 \\
\hline & Transfer of & 40.61 & 20.90 \\
\hline & control & & \\
\hline
\end{tabular}

\section{Accuracy of Reported Details}

The accuracy of reports was assessed via the previously described coding categories: accurate, inaccurate, subjective, non-scoreable. Each category was assessed in two ways. First, the quantity (i.e., number) of accurate, inaccurate, subjective, nonscoreable, and repeated details were compared across conditions. Second, the proportions 
of each category of detail were compared across conditions. Proportions were calculated by dividing the number of details in a given category by the total number of units of information given by the participant. For example, the proportion of accurate details was calculated as (number of accurate details given by participant) / (number of total units of information given by participant). Accuracy rates were calculated for the first free recall question, second experimental question, and final follow-up question.

Reverse order condition. The critical comparison for the reverse order mnemonic is between the second recall attempts in the control and reverse order conditions. In the control condition, participants were asked to tell the experimenter again everything they could remember; in the reverse order condition, participants were asked to recount their memory in reverse order.

Quantity of accurate details. A $2 \times 2$ ANOVA, including interview condition (control, reverse order) and proficiency level (native, NNES), was used to detect mean differences on quantity of accurate details reported in the second recall attempt. Because details scored as repeated were not further scored for accuracy, quantity of accurate details includes only the number of unique accurate units provided by participants. There was a significant main effect of interview condition, $F(1,51)=8.02, p=.007$, partial $\eta^{2}=$ .14. Participants in the control condition $(M=19.82, S D=17.86)$ provided significantly more accurate details in their second recall attempt than participants in the reverse order condition $(M=8.04, S D=5.74)$. There was also a significant main effect of language proficiency, $F(1,51)=6.03, p=.017$, partial $\eta^{2}=.11$. Native English speakers $(M=$ $17.26, S D=16.64)$ reported significantly more accurate details in their second recall attempt than NNESs $(M=8.40, S D=7.13)$. The main effects were qualified by a 
marginally significant interaction, $F(1,51)=3.26, p=.077$, partial $\eta^{2}=.06$. Pairwise comparisons revealed that native English speakers reported more accurate details in response to the second interview question in the control condition $(M=25.17, S D=$ 19.64) compared to the reverse order condition $(M=8.88, S D=5.83), p<.001$. However, the difference in accurate details between interview conditions was not significant for NNES participants $\left(M_{\text {control }}=10.20, S D_{\text {control }}=8.31 ; M_{\text {reverse }}=6.60\right.$, $\left.S D_{\text {reverse }}=5.58\right)$. The statistical interaction is graphed in Figure 10 .

Accurate units of information reported to the second (experimental) interview question

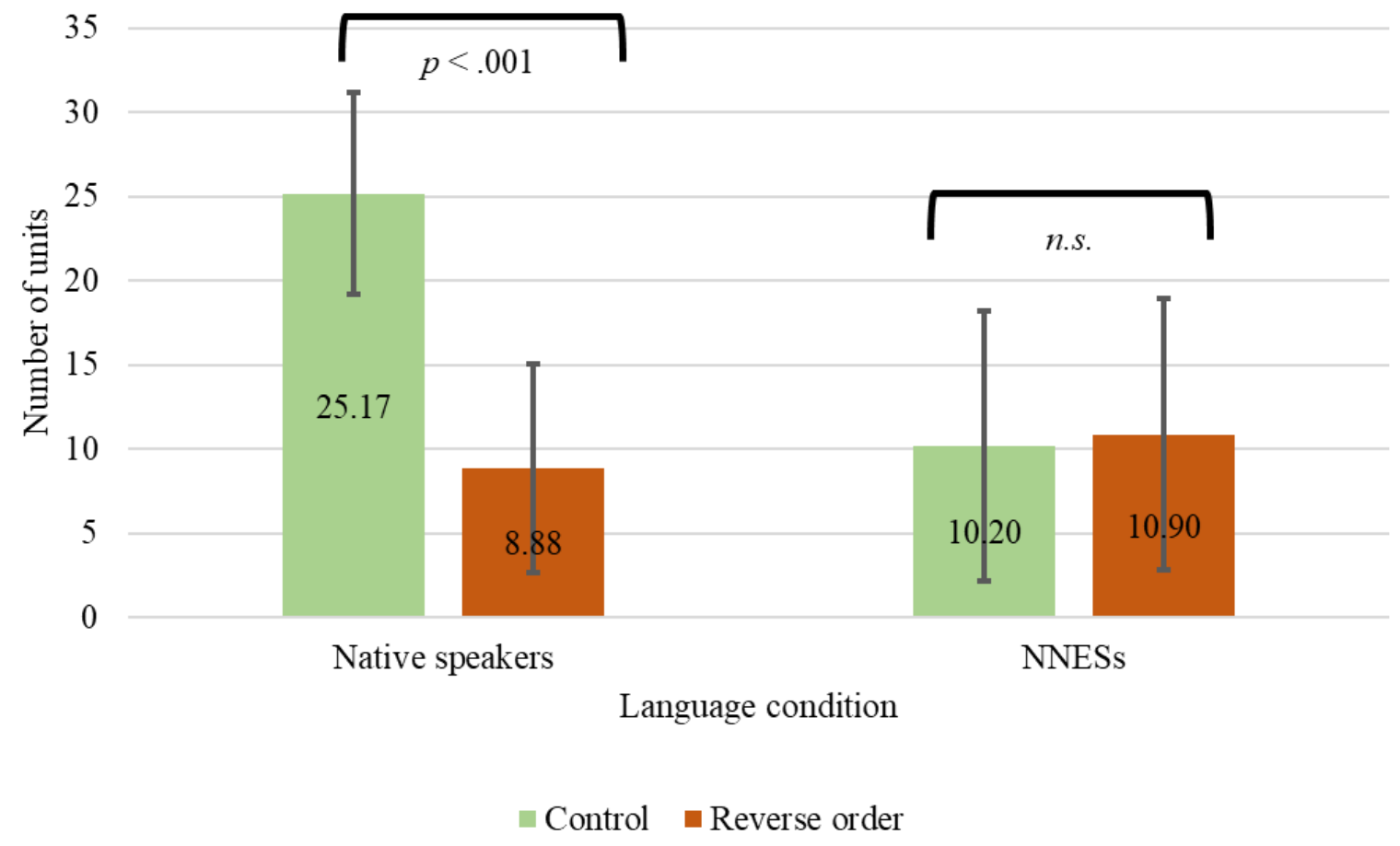

Figure 10. Estimated marginal means and 95\% confidence intervals for units of information reported to the second (experimental) interview question (control vs. reverse order conditions). 
Proportion of accurate details. A $2 \times 2$ ANOVA, including interview condition (control, reverse order) and proficiency level (native, NNES), was used to detect mean differences in proportions of accurate details reported in the second recall attempt. There was no main effect of interview condition, $F(1,51)=1.59, p=.214$, partial $\eta^{2}=.03$. There was also no main effect of language, $F(1,51)=.15, p=.699$, partial $\eta^{2}<.01$. There was no significant interaction, $F(1,51)=.10, p=.754$, partial $\eta^{2}<.01$. See Table 6 for descriptive statistics.

Table 6. Descriptive statistics for accurate details (control vs. reverse order conditions).

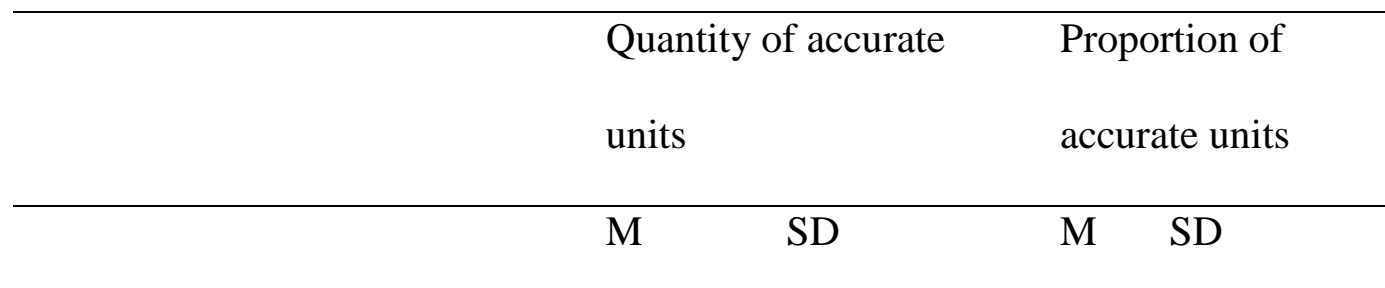

Native

$\begin{array}{lcccc}\text { Control } & 25.17^{\mathrm{b}} & 19.64 & .45 & .23 \\ \text { Reverse order } & 8.88^{\mathrm{b}} & 5.83 & .34 & .22 \\ \text { Total } & 17.26 & 16.64 & .40 & .23\end{array}$

NNES

$\begin{array}{lcccc}\text { Control } & 10.20 & 8.31 & .41 & .31 \\ \text { Reverse order } & 10.90 & 7.19 & .46 & .25 \\ \text { Total } & 10.55 & 7.57 & .43 & .27\end{array}$

All participants

$\begin{array}{lllll}\text { Control } \quad 19.82^{\mathrm{a}} & 17.86 & .44 & .26\end{array}$


Note. Means sharing a superscript letter indicate significant differences. ${ }^{\mathrm{a}}$ Main effect of interview condition, $p=033$. ${ }^{\mathrm{b}}$ Interview $\times$ language interaction, pairwise $p<.001$.

Quantity of inaccurate details. A $2 \times 2$ ANOVA, including interview condition (control, reverse order) and proficiency level (native, NNES), was used to detect mean differences in quantity of inaccurate details reported in the second recall attempt. There was no significant main effect of interview condition, $F(1,51)=2.27, p=.138$, partial $\eta^{2}$ $=.04$. There was also no significant main effect of language, $F(1,51)=0.09, p=.769$, partial $\eta^{2}=.00$. There was no significant interaction, $F(1,51)=.04, p=.834$, partial $\eta^{2}<$ .01. See Table 7 for descriptive statistics.

Proportion of inaccurate details. A $2 \times 2$ ANOVA, including interview condition (control, reverse order) and proficiency level (native, NNES), was used to detect mean differences in proportion of inaccurate details reported in the second recall attempt. There was no significant main effect of interview condition, $F(1,51)=0.49, p=.487$, partial $\eta^{2}=.01$. There was also no significant main effect of language, $F(1,51)=0.31, p$ $=.577$, partial $\eta^{2}=.01$. There was no significant interaction, $F(1,51)=1.82, p=.183$, partial $\eta^{2}=.04$. See Table 7 for descriptive statistics. 
Table 7. Descriptive statistics for inaccurate details (control vs. reverse order conditions).

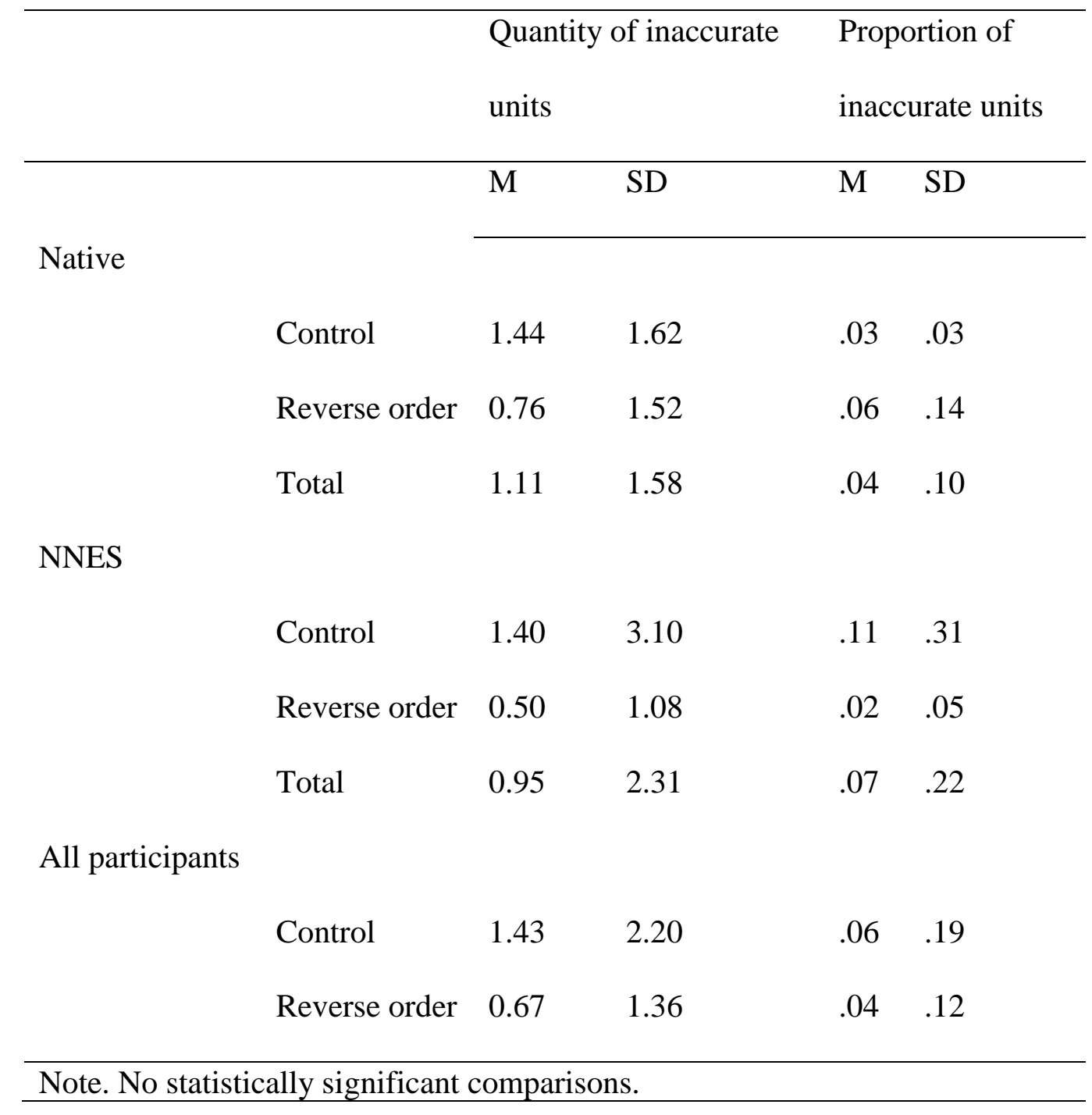

Quantity of subjective details. A $2 \times 2$ ANOVA, including interview condition (control, reverse order) and proficiency level (native, NNES), was used to detect mean differences in quantity of subjective details reported in the second recall attempt. There was a significant main effect of interview condition, $F(1,51)=4.48, p=.039$, partial $\eta^{2}=$ .08. Participants in the control condition $(M=1.07, S D=1.51)$ provided significantly more subjective details than participants in the reverse order condition $(M=0.44, S D=$ 
0.70). There was no significant main effect of language, $F(1,51)=.43, p=.514$, partial $\eta^{2}=.01$. There was no significant interaction, $F(1,51)=.75, p=.389$, partial $\eta^{2}=.02$. See Table 8 for descriptive statistics.

Proportion of subjective details. A $2 \times 2$ ANOVA, including interview condition (control, reverse order) and proficiency level (native, NNES), was used to detect mean differences in proportion of subjective details reported in the second recall attempt. There was a marginally significant effect of interview condition, $F(1,51)=3.84, p=.056$, partial $\eta^{2}=.07$. Participants in the control interview condition $(M=.03, S D=.05)$ reported a somewhat higher proportion of subjective details than participants in the reverse order condition $(M=.02, S D=.03)$. There was also a significant effect of language, $F(1,51)=4.28, p=.044$, partial $\eta^{2}=.08$. Native English speakers $(M=.01$, $S D=.03)$ reported a lower proportion of subjective details than NNESs $(M=.03, S D=$ $.05)$. The main effects were qualified by a significant interaction, $F(1,51)=5.25, p=$ .026 , partial $\eta^{2}=.09$. Pairwise comparisons revealed that NNESs reported a higher proportion of subjective details in response to the second interview question in the control condition $(M=.06, S D=.06)$ compared to the reverse order condition $(M=.01$, $S D=.02), p<.001$. However, the difference in proportion of subjective details between interview conditions was not significant for native English speakers $\left(M_{\text {control }}=.01\right.$, $\left.S D_{\text {control }}=.02 ; M_{\text {reverse }}=.02, S D_{\text {reverse }}=.03\right)$. The statistical interaction is graphed in Figure 11. See Table 8 for descriptive statistics. 
Table 8. Descriptive statistics for subjective units (control vs. reverse order conditions).

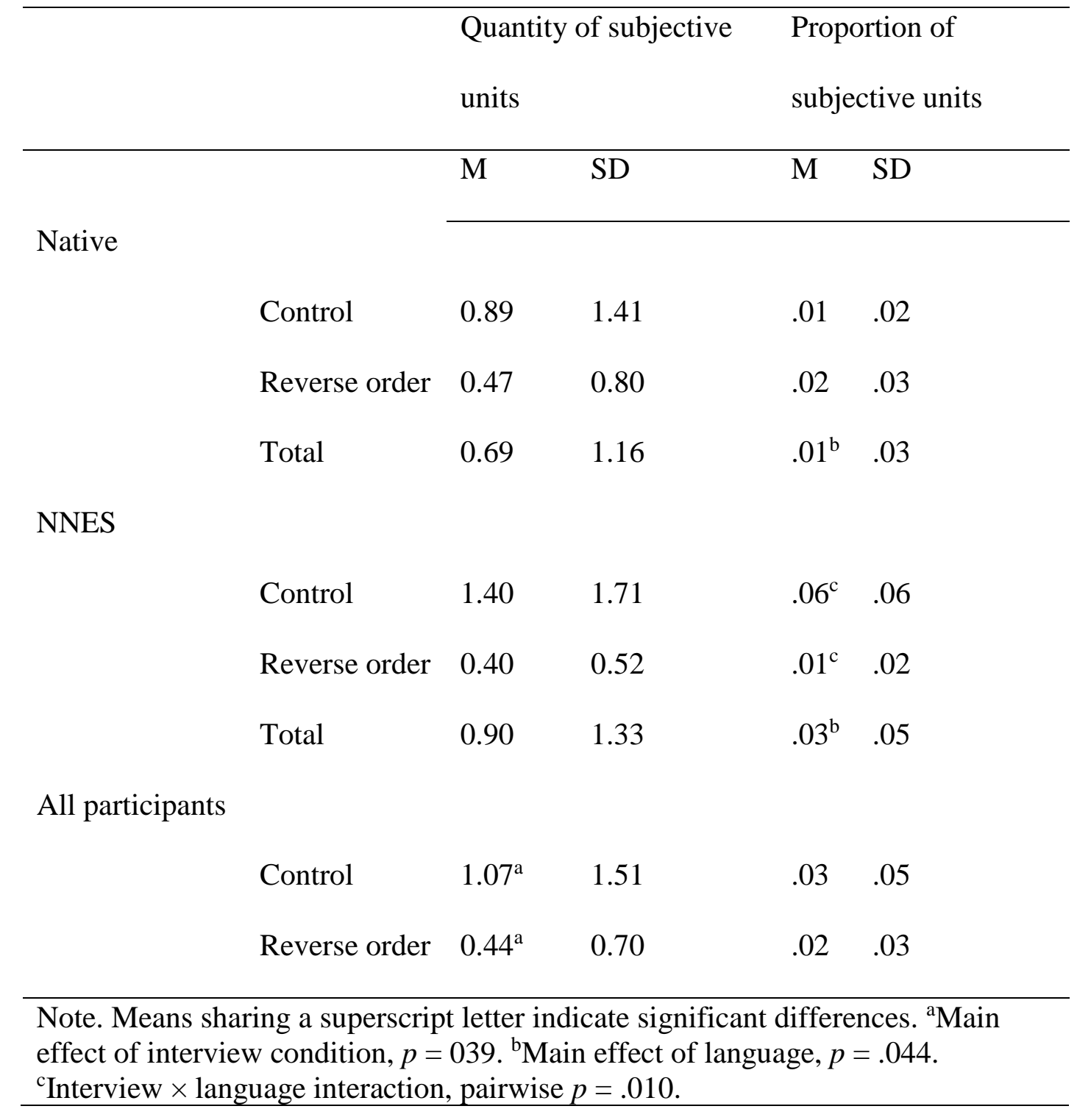


Proportion of subjective units of information reported to the second (experimental) interview question

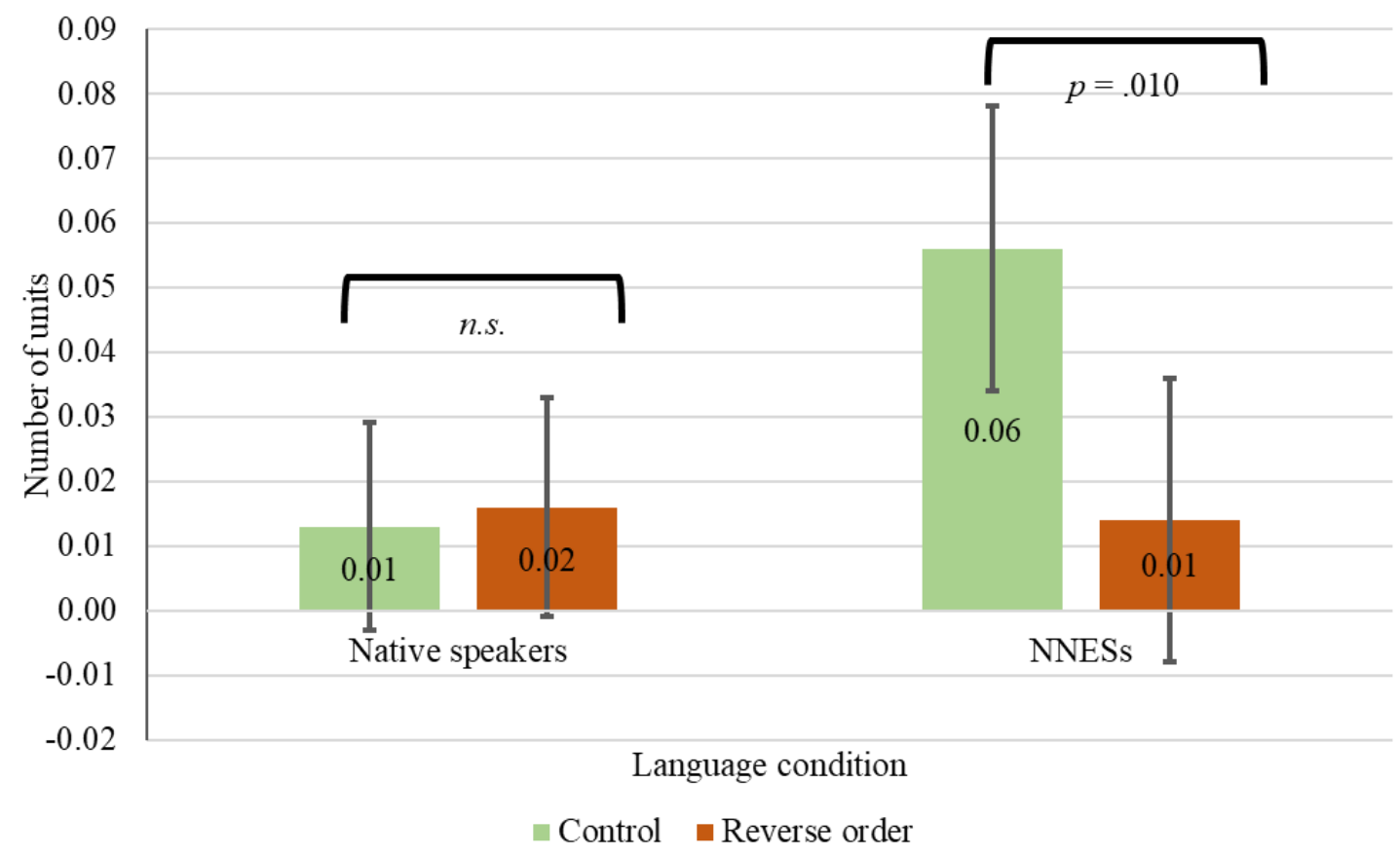

Figure 11. Estimated marginal means and 95\% confidence intervals for proportion of subjective units of information reported to the second interview question (control vs. reverse order conditions).

Quantity of non-scoreable details. A $2 \times 2$ ANOVA, including interview condition (control, reverse order) and proficiency level (native, NNES), was used to detect mean differences in quantity of non-scoreable details reported in the second recall attempt. There was no significant main effect of interview condition, $F(1,51)=0.12, p=.744$, partial $\eta^{2}<.01$. There was also no significant main effect of language, $F(1,51)=1.56, p$ $=.218$, partial $\eta^{2}=.03$. There was also no significant interaction, $F(1,51)=0.06, p=$ .806, partial $\eta^{2}<.01$

Proportion of non-scoreable details. A $2 \times 2$ ANOVA, including interview condition (control, reverse order) and proficiency level (native, NNES), was used to 
detect mean differences in proportion of non-scoreable details reported in the second recall attempt. There was no significant main effect of interview condition, $F(1,51)=$ $1.47, p=.231$, partial $\eta^{2}=.03$. There was also no significant main effect of language, $F(1,51)=0.00, p=.999$, partial $\eta^{2}<.01$. There was also no significant interaction, $F(1$, 51) $=0.00, p=.963$, partial $\eta^{2}<.01$.

Table 9. Descriptive statistics for non-scoreable units (control vs. reverse order conditions).

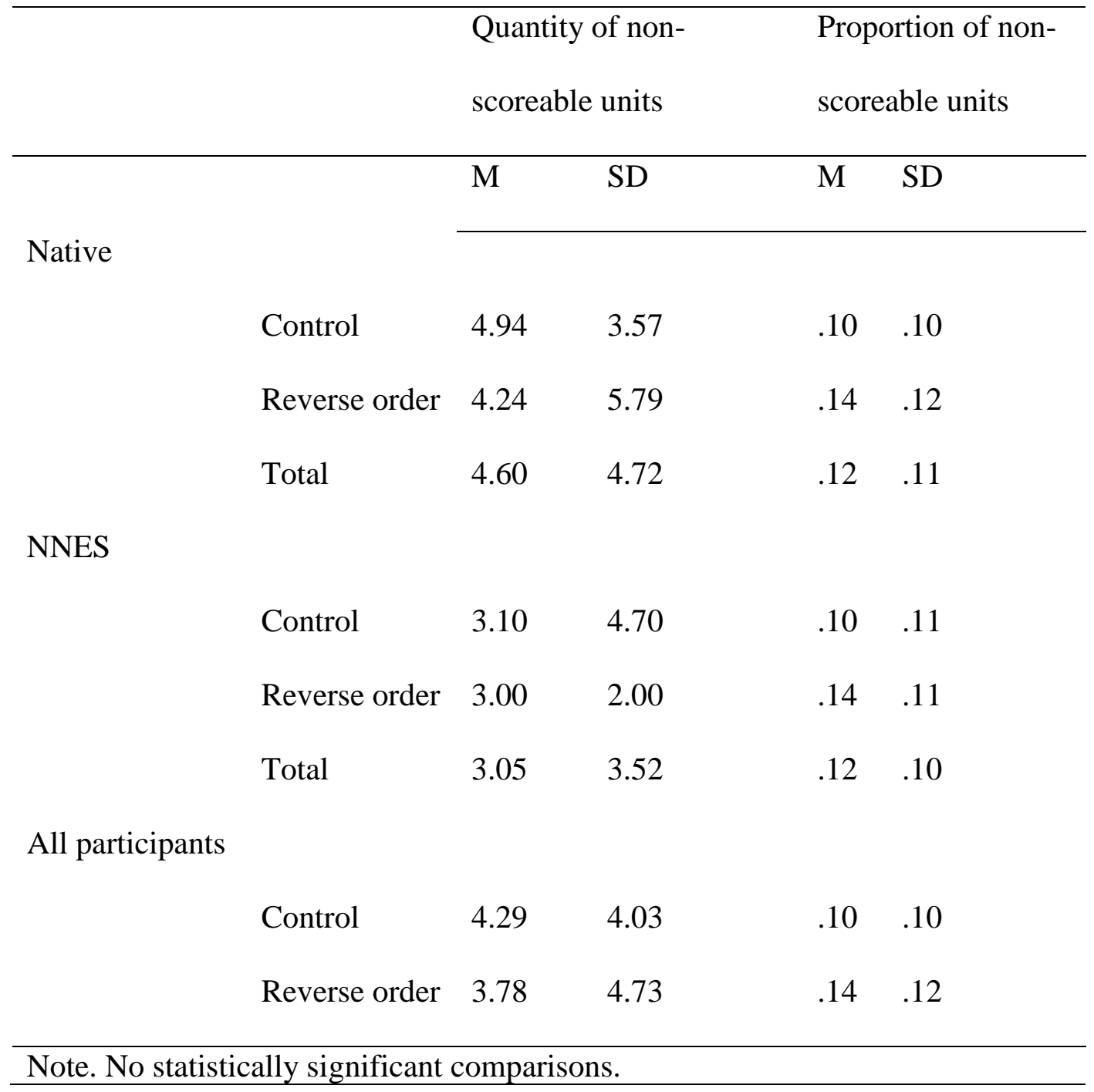


Quantity of repeated details. A $2 \times 2$ ANOVA, including interview condition (control, reverse order) and proficiency level (native, NNES), was used to detect mean differences in quantity of repeated details reported in the second recall attempt. There was no significant main effect of interview condition, $F(1,51)=1.54, p=.220$, partial $\eta^{2}$ $=.03$. There was also no significant main effect of language, $F(1,51)=1.17, p=.285$, partial $\eta^{2}=.02$. There was also no significant interaction, $F(1,51)=2.11, p=.152$, partial $\eta^{2}=.04$.

Proportion of repeated details. A $2 \times 2$ ANOVA, including interview condition (control, reverse order) and proficiency level (native, NNES), was used to detect mean differences in proportion of repeated details reported in the second recall attempt. There was no significant main effect of interview condition, $F(1,51)=.28, p=.599$, partial $\eta^{2}$ $=.01$. There was also no significant main effect of language, $F(1,51)=1.08, p=.303$, partial $\eta^{2}=.02$. There was also no significant interaction, $F(1,51)=0.00, p=.970$, partial $\eta^{2}<.01$.

Table 10. Descriptive statistics for repeated units (control vs. reverse order conditions).

\begin{tabular}{llllll}
\hline & & \multicolumn{2}{l}{$\begin{array}{l}\text { Quantity of repeated } \\
\text { units }\end{array}$} & \multicolumn{2}{c}{$\begin{array}{l}\text { Proportion of } \\
\text { repeated units }\end{array}$} \\
\hline \multirow{3}{*}{ Native } & M & SD & M & SD \\
\cline { 3 - 6 } & & & & & \\
& Control & 23.33 & 23.70 & .40 & .22 \\
& Reverse order & 10.65 & 7.31 & .44 & .27 \\
& Total & 17.17 & 18.64 & .42 & .24
\end{tabular}


NNES

$\begin{array}{lcccc}\text { Control } & 11.40 & 15.17 & .32 & .33 \\ \text { Reverse order } & 12.40 & 14.52 & .36 & .28 \\ \text { Total } & 11.90 & 14.46 & .34 & .30\end{array}$

All participants

$\begin{array}{lllll}\text { Control } & 19.07 & 21.55 & .37 & .26 \\ \text { Reverse order } & 11.30 & 10.32 & .41 & .27\end{array}$

Note. No statistically significant comparisons.

Transfer of control condition. The critical comparison for the transfer of control instruction is between the first recall attempts in the control and instructions conditions. Both recall attempts were open-ended free recall prompts; in the transfer of control condition, participants also heard the transfer of control instruction in addition to the control instructions.

Quantity of accurate details. A $2 \times 2$ ANOVA, including interview condition (control, transfer of control) and proficiency level (native, NNES), was used to detect mean differences in quantity of accurate details reported in the first recall attempt. Because details scored as repeated were not further scored for accuracy, quantity of accurate details includes only the number of unique accurate units provided by participants. There was no significant main effect of interview condition, $F(1,58)=0.54$, $p=.464$, partial $\eta^{2}=.01$. There was a significant main effect of language, $F(1,58)=$ 5.92, $p=.018$, partial $\eta^{2}=.09$. Native English speakers $(M=30.79, S D=14.00)$ reported significantly more accurate details than NNESs $(M=22.09, S D=12.00)$. There was no 
significant interaction, $F(1,58)=0.12, p=.731$, partial $\eta^{2}<.01$. See Table 11 for descriptive statistics.

Proportion of accurate details. A $2 \times 2$ ANOVA, including interview condition (control, transfer of control) and proficiency level (native, NNES), was used to detect mean differences in quantity of accurate details reported in the first recall attempt. There was no significant main effect of interview condition, $F(1,58)=0.00, p=.970$, partial $\eta^{2}$ $<.01$. There was also no significant main effect of language, $F(1,58)=1.03, p=.314$, partial $\eta^{2}=.02$. There was no significant interaction, $F(1,58)=0.13, p=.722$, partial $\eta^{2}$ $<.01$. See Table 11 for descriptive statistics.

Table 11. Descriptive statistics for accurate details (control vs. transfer of control conditions).

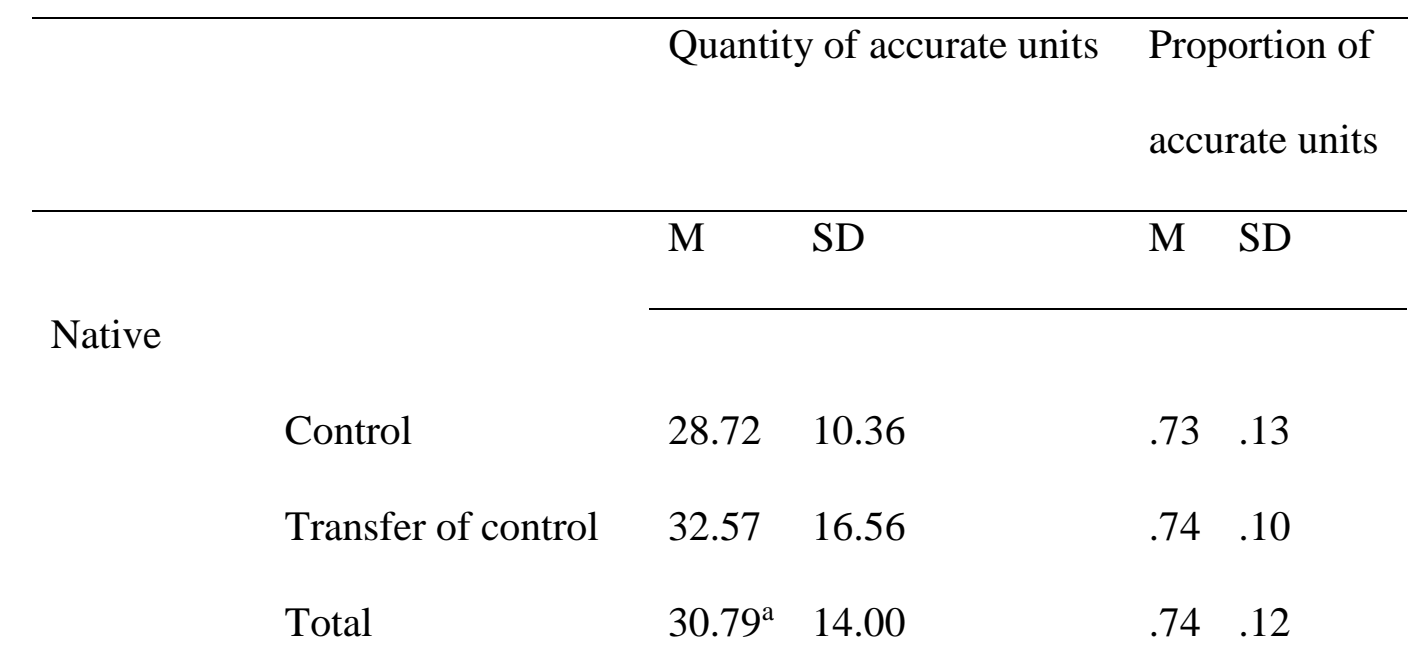

NNES

$\begin{array}{lclcc}\text { Control } & 21.30 & 14.86 & .70 & .28 \\ \text { Transfer of control } & 22.69 & 9.88 & .69 & .13 \\ \text { Total } & 22.09^{\mathrm{a}} & 12.00 & .69 & .20\end{array}$


All

participants
Control
26.07
12.42
$.72 \quad .19$
Transfer of control
$28.79 \quad 15.01$
$.72 \quad .11$

Note. Means sharing a superscript letter indicate significant differences. ${ }^{\mathrm{a}}$ Main effect of language, $p=.018$.

Quantity of inaccurate details. A $2 \times 2$ ANOVA, including interview condition (control, transfer of control) and proficiency level (native, NNES), was used to detect mean differences in quantity of inaccurate details reported in the first recall attempt. There was no significant main effect of interview condition, $F(1,58)=0.31, p=.580$, partial $\eta^{2}=.01$. There was also no significant main effect of language, $F(1,58)=2.63, p$ $=.111$, partial $\eta^{2}=.04$. There was no significant interaction, $F(1,58)=0.12, p=.727$, partial $\eta^{2}<.01$

Proportion of inaccurate details. A $2 \times 2$ ANOVA, including interview condition (control, transfer of control) and proficiency level (native, NNES), was used to detect mean differences in proportion of inaccurate details reported in the first recall attempt. There was no significant main effect of interview condition, $F(1,58)=0.63, p=.432$, partial $\eta^{2}=.01$. There was also no significant main effect of language, $F(1,58)=1.27, p$ $=.264$, partial $\eta^{2}=.02$. There was no significant interaction, $F(1,58)=0.34, p=.561$, partial $\eta^{2}=.01$ 
Table 12. Descriptive statistics for inaccurate details (control vs. transfer of control conditions).

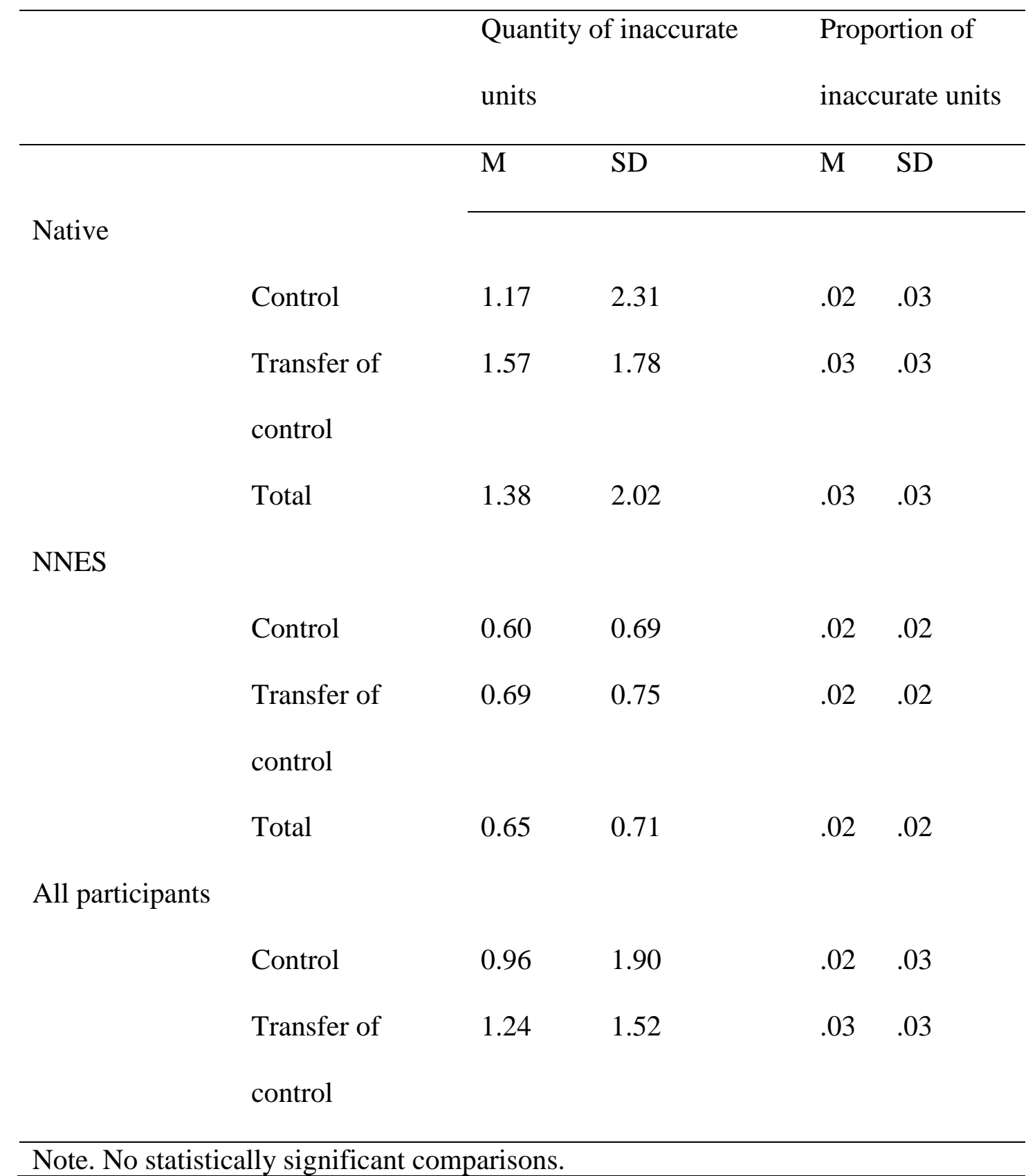

Quantity of subjective details. A $2 \times 2$ ANOVA, including interview condition (control, transfer of control) and proficiency level (native, NNES), was used to detect mean differences in quantity of subjective details reported in the first recall attempt. 
There was no significant main effect of interview condition, $F(1,58)=0.17, p=.686$, partial $\eta^{2}<.01$. There was also no significant main effect of language, $F(1,58)=0.17, p$ $=.686$, partial $\eta^{2}<.01$. There was no significant interaction, $F(1,58)=0.06, p=.804$, partial $\eta^{2}<.01$. See Table 13 for descriptive statistics.

Proportion of subjective details. A $2 \times 2$ ANOVA, including interview condition (control, transfer of control) and proficiency level (native, NNES), was used to detect mean differences in proportion of subjective details reported in the first recall attempt. There was no significant main effect of interview condition, $F(1,58)=0.14, p=.713$, partial $\eta^{2}<.01$. There was a marginally significant main effect of language, $F(1,58)=$ $3.12, p=.083$, partial $\eta^{2}=.05$. Non-native English speakers $(M=.07, S D=.09)$ reported somewhat higher proportions of subjective details to the first question than native English speakers $(M=.04, S D=.05)$. There was no significant interaction, $F(1,58)=0.04, p=$ .846, partial $\eta^{2}<.01$. See Table 13 for descriptive statistics.

Table 13. Descriptive statistics for subjective details (control vs. transfer of control conditions).

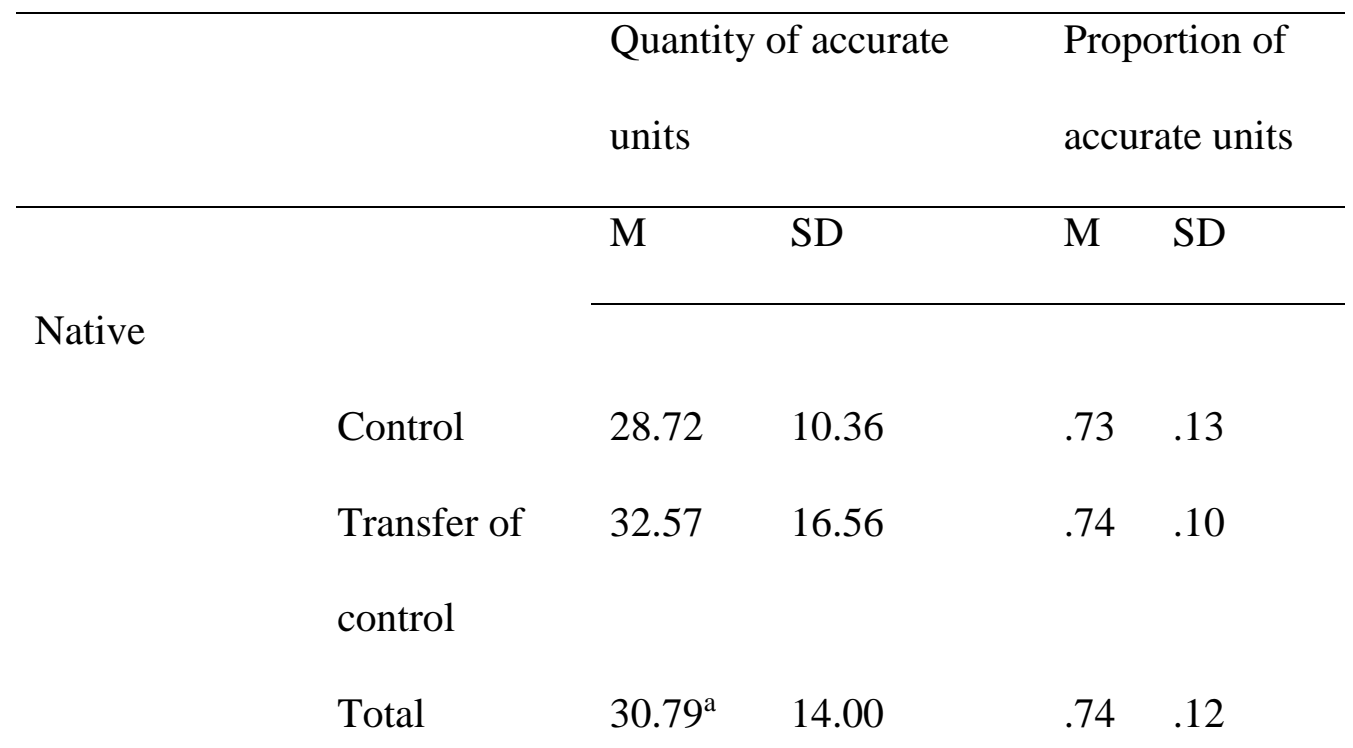


$1.50, p=.226$, partial $\eta^{2}=.03$. There was no significant interaction, $F(1,58)=0.46, p=$ .502 , partial $\eta^{2}=.01$. See Table 14 for descriptive statistics.

Table 14. Descriptive statistics for non-scoreable details (control vs. transfer of control conditions).

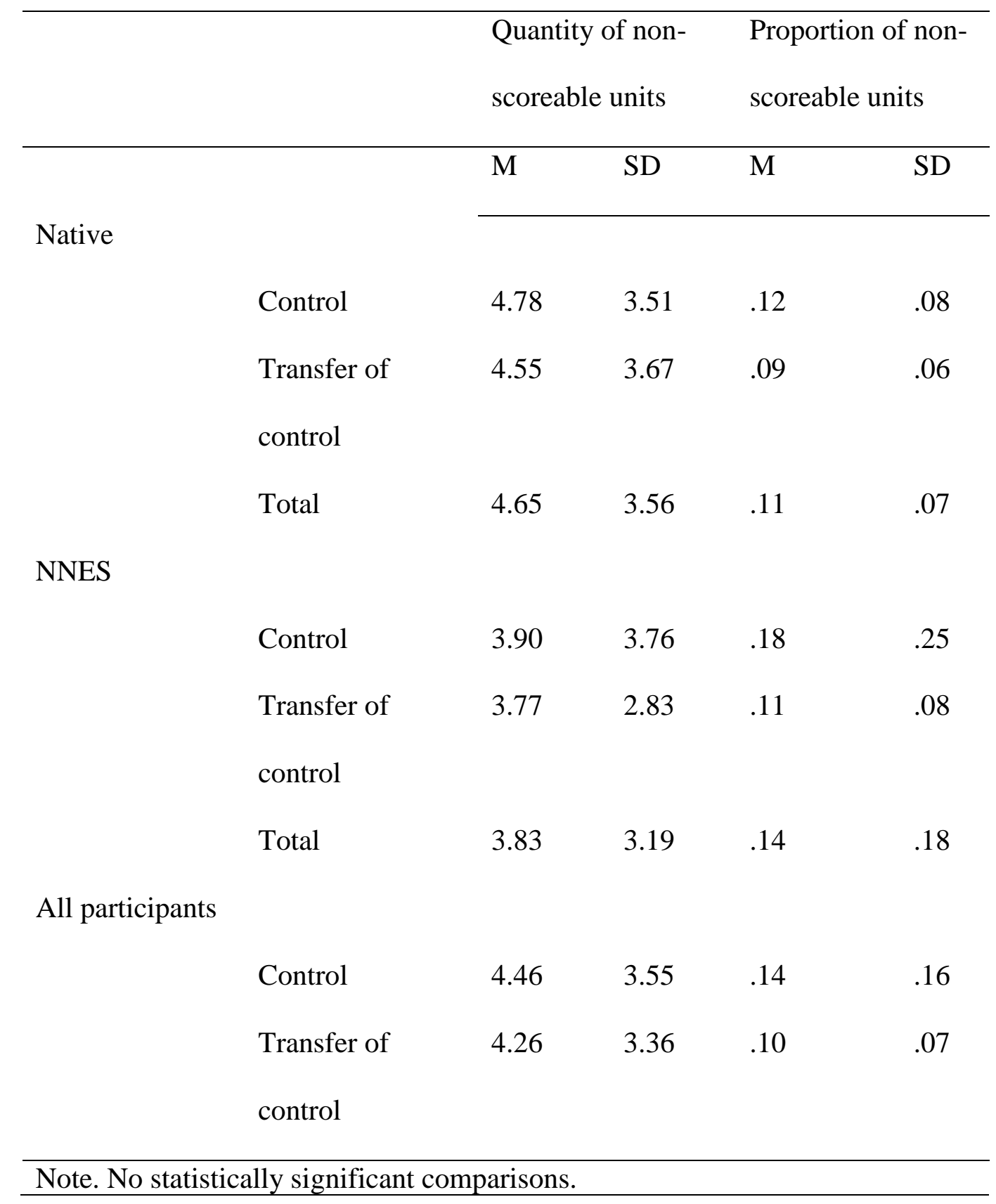


Quantity of repeated details. A $2 \times 2$ ANOVA, including interview condition (control, transfer of control) and proficiency level (native, NNES), was used to detect mean differences in quantity of repeated details reported in the first recall attempt. There was no significant main effect of interview condition, $F(1,58)=1.40, p=.242$, partial $\eta^{2}$ $=.02$. There was a marginally significant main effect of language, $F(1,58)=3.28, p=$ .075 , partial $\eta^{2}=.05$. Native English speakers $(M=4.03, S D=5.04)$ included somewhat more repeated details than NNESs $(M=2.13, S D=2.47)$. There was no significant interaction, $F(1,58)=1.10, p=.298$, partial $\eta^{2}=.02$.

Proportion of repeated details. A $2 \times 2$ ANOVA, including interview condition (control, transfer of control) and proficiency level (native, NNES), was used to detect mean differences in proportion of repeated details reported in the first recall attempt. There was a significant main effect of interview condition, $F(1,58)=5.31, p=.025$, partial $\eta^{2}=.08$. Participants in the transfer of control condition $(M=.10, S D=.08)$ reported a significantly higher proportion of repeated details compared to participants in the control condition $(M=.06, S D=.08)$. There was no significant main effect of language, $F(1,58)=.85, p=.360$, partial $\eta^{2}=.01$. There was a marginally significant interaction, $F(1,58)=3.12, p=.082$, partial $\eta^{2}=.05$. Pairwise comparisons indicate NNESs reported somewhat more repeated details in the transfer of control condition $(M=$ $.11, S D=.10)$ compared to the control condition $(M=.02, S D=.03), p=.013$; there was no difference between the conditions for native English speakers $\left(M_{\text {control }}=.08, S D_{\text {control }}=\right.$ $\left..09 ; M_{\text {transfer of control }}=.09, S D_{\text {transfer of control }}=.08\right)$. The interaction is graphed in Figure 12. 
Table 15. Descriptive statistics for repeated details (control vs. transfer of control conditions).

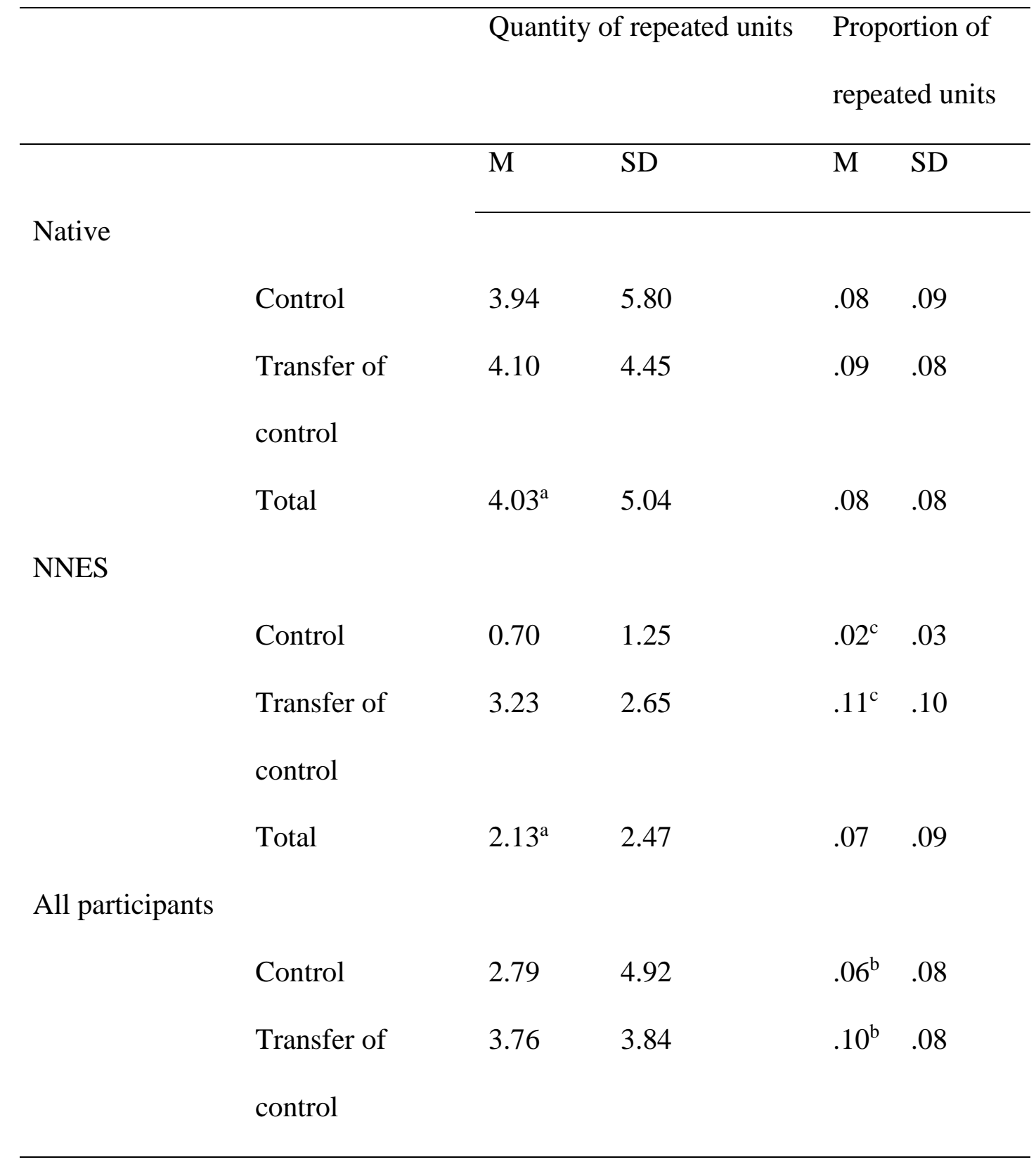

Note. Means sharing a superscript letter indicate marginally or statistically significant differences. ${ }^{\mathrm{a}}$ Marginal main effect of language, $p=.075$. ${ }^{\mathrm{b}}$ Main effect of interview condition, $p=.025$. $^{\mathrm{c}}$ Marginally significant interaction, omnibus $p=$ .082 ; pairwise $p=.013$. 


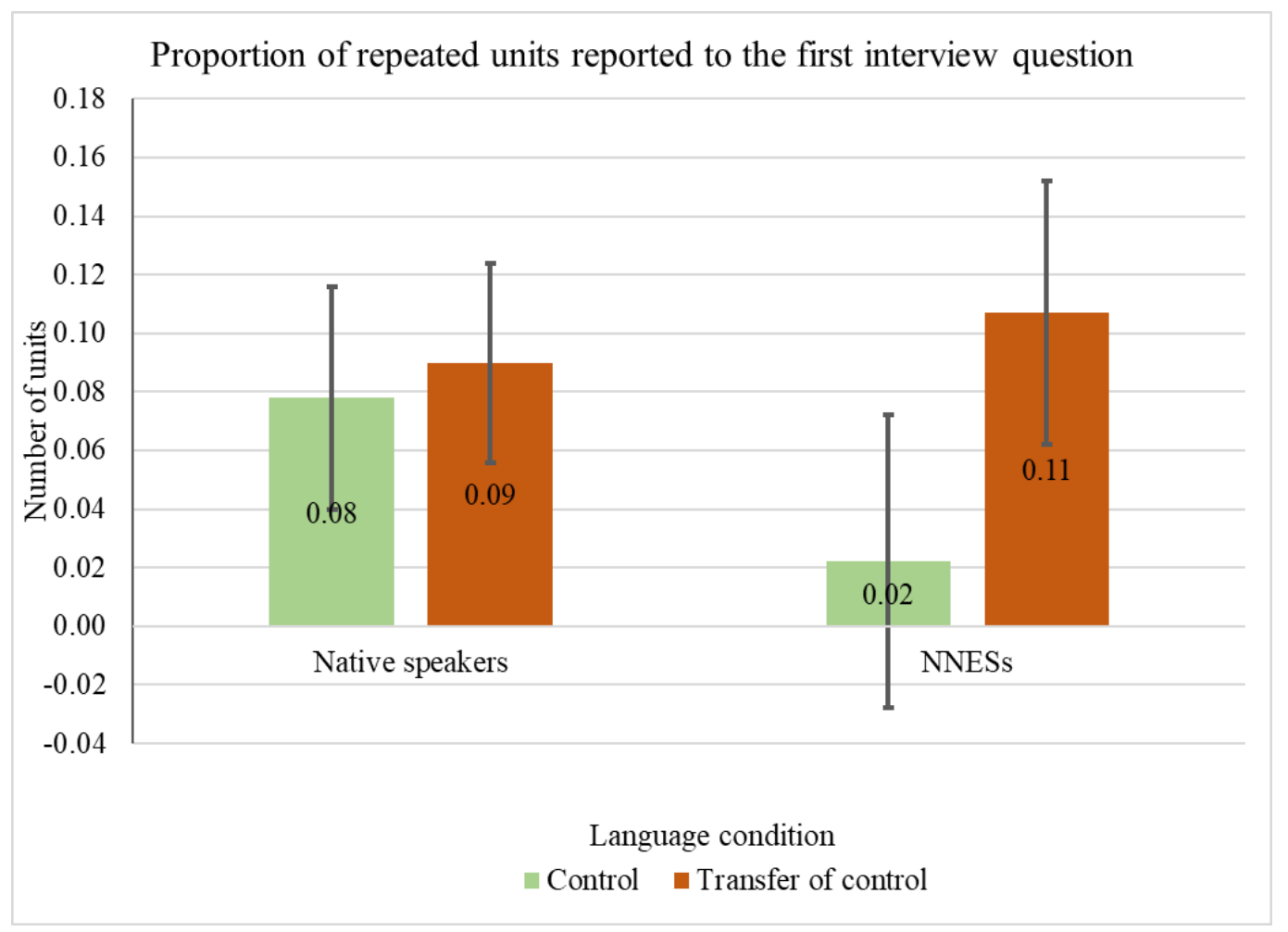

Figure 12. Estimated marginal means and $95 \%$ confidence intervals for proportion of repeated details reported in the first interview question (control vs. transfer of control conditions)

\section{Accuracy Rates Across Interview Questions}

A two-way mixed ANOVA was used to assess whether accuracy rates differed between the first, open-ended recall question and the second, experimental question. A 2 $\times 2$ repeated measures ANOVA was conducted with interview condition (control, reverse order) and proficiency level (native, NNES) as between-participant factors and proportion of accurate details ${ }^{2}$ (question 1, question 2) as a within-participants variable. There was no significant difference between accuracy rates for question 1 and question 2, $F(1,53)=1.67, p=.201$, partial $\eta^{2}=.03$. There was no significant interaction between

\footnotetext{
${ }^{2}$ For the two-way mixed ANOVA, proportion of accurate details was calculated as number of accurate details divided by number of accurate, inaccurate, and subjective details for each interview question. Unscorable and repeated details were not included.
} 
accuracy and language condition, $F(1,53)<.01, p=.952$, partial $\eta^{2}<.01$, nor between accuracy and interview condition, $F(1,53)=.34, p=.563$, partial $\eta^{2}=.01$. Finally, the three-way interaction between accuracy, language, and interview condition was not significant, $F(1,53)=.63, p=.432$, partial $\eta^{2}=.03$. See Table 16 for descriptive statistics.

Table 16. Descriptive statistics for between-participants analysis of proportion of accurate details (control vs. reverse order condition).

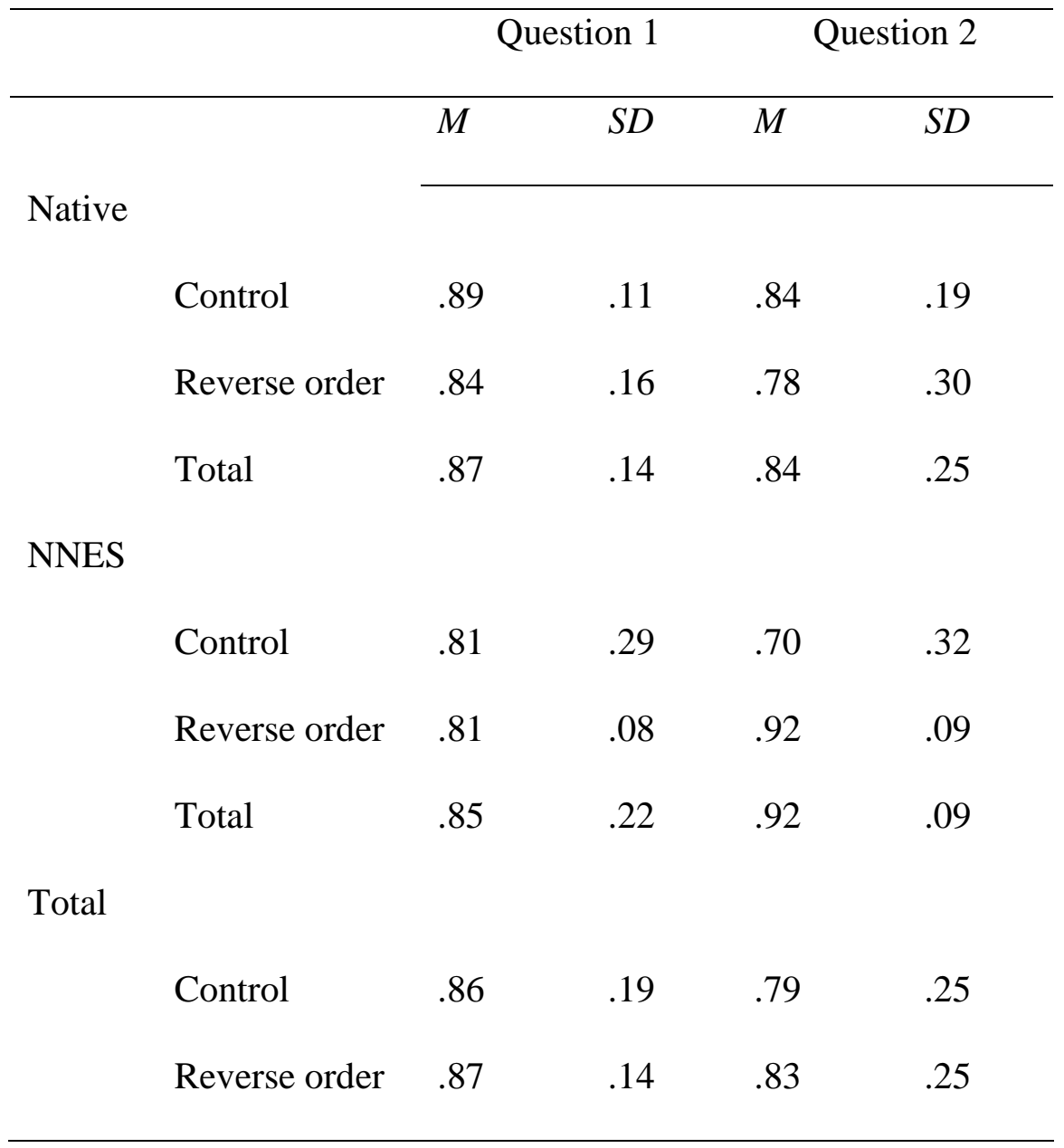




\section{New Information}

The benefit of the reverse order technique (over the control, repeated interview condition) may manifest in terms of new information reported. That is, does the reverse order technique increase amount or accuracy of new information (i.e., information not previously reported to the first open-ended recall) relative to the control interview? New information was calculated for the experimental interview question in the control and reverse order conditions; new information was calculated as the sum of accurate, inaccurate, and subjective details. Repeated details and non-scoreable details were not considered new information.

A $2 \times 2$ ANOVA, including interview condition (control, reverse order) and proficiency level (native, NNES), was used to detect mean differences in number of new details reported in the second recall attempt. There was no significant main effect of language, $F(1,51)=1.94, p=.169$, partial $\eta^{2}=.03$. There was a significant main effect of interview condition, $F(1,51)=5.43, p=.023$, partial $\eta^{2}=.09$. Participants in the control condition $(M=27.03, S D=25.12)$ reported significantly more new information during the second recall attempt than participants in the reverse order condition $(M=$ $13.14, S D=11.52)$. There was no significant interaction, $F(1,51)=1.02, p=.318$, partial $\eta^{2}=.02$. 
Table 17. Descriptive statistics for quantity of new details reported to the second interview question (control vs. reverse order analysis).

\begin{tabular}{|c|c|c|c|}
\hline & & \multicolumn{2}{|c|}{ New details } \\
\hline & & $M$ & $S D$ \\
\hline \multicolumn{4}{|l|}{ Native } \\
\hline & Control & 31.55 & 26.68 \\
\hline & Reverse order & 13.84 & 13.35 \\
\hline & Total & 22.92 & 22.82 \\
\hline \multicolumn{4}{|l|}{ NNES } \\
\hline & Control & 18.82 & 20.61 \\
\hline & Reverse order & 11.80 & 7.32 \\
\hline & Total & 15.48 & 15.79 \\
\hline \multicolumn{4}{|l|}{ Total } \\
\hline & Control & 27.03 & 25.12 \\
\hline & Reverse order & 13.14 & 11.52 \\
\hline
\end{tabular}

\section{English Proficiency and Quantity of Detail}

Units of information. Correlations were computed between participants' reported English proficiency ratings and number of details recalled. Correlational analyses revealed a significant correlation between participants' reported ability to speak English and the quantity of details given in the first (free recall) interview question, $r(87)=.24, p$ $=.047$. Participants who reported higher levels of English speaking ability also reported 
more details during the first interview question. Additionally, participants' reported ability to write in English was significantly correlated with number of details given in the first interview question, $r(87)=.22, p=.033$. Participants who reported higher levels of English writing ability also reported more details during the first interview question. Other correlations were not significant. See Table 18 for a full report of correlational analyses.

Table 18. Correlations between participants' reported English proficiency and number of details given.

\begin{tabular}{|c|c|c|c|c|c|c|}
\hline & \multicolumn{2}{|c|}{ First question } & \multicolumn{2}{|c|}{ Experimental question } & \multicolumn{2}{|c|}{ Total interview } \\
\hline English ability & $r(n=87)$ & $p$ & $r(n=59)$ & $p$ & $r(n=59)$ & $p$ \\
\hline Listening & .09 & .430 & -.04 & .992 & .001 & .992 \\
\hline Speaking & .21 & .047 & .05 & .668 & .11 & .409 \\
\hline Reading & .13 & .222 & -.16 & .223 & -.07 & .563 \\
\hline Writing & .23 & .033 & .00 & .992 & .10 & .469 \\
\hline \multicolumn{7}{|c|}{$\begin{array}{l}\text { Note: Details in total interview are calculated by summing the details reported to the } \\
\text { first question and experimental question; sample sizes are smaller in the } \\
\text { experimental question column and total interview column because the transfer of } \\
\text { control condition does not include an experimental question. Sample size for these } \\
\text { analyses is smaller than the whole sample because of incomplete LHQ data for these } \\
\text { questions. }\end{array}$} \\
\hline
\end{tabular}




\section{DISCUSSION}

The present dissertation aimed to test the utility of two mnemonics from the CIwhich are widely regarded as best practice eyewitness interviewing techniques—with a potentially vulnerable population. Non-native English speakers face a cognitively taxing task when they provide a statement in their non-native language, and some of the CI mnemonics are cognitively demanding. Moreover, NNESs may not have sufficient cognitive resources to engage in the CI mnemonics while formulating a statement in their second language. The increase in complexity (between speaking a non-native language and manipulating that response into an unusual order) may result in a decrease in completeness and/or accuracy. The tradeoff between complexity and completeness/accuracy is concerning because eyewitness evidence is often a critical component of criminal investigations and incomplete eyewitness testimony can impede criminal investigations and the administration of justice. However, some components of the CI, like introductory instructions that encourage witnesses to report as much as they can, may be especially helpful with NNESs. Previous research suggests NNESs may be less forthcoming in general; encouragement from the interviewer may help them overcome their hesitation to report details.

\section{Effects of Language}

Units of information. In general, results indicated significant and marginally significant effects of language proficiency on units of information provided by participants. Unsurprisingly, NNESs tended to provide fewer details than native English speakers. The finding is consistent with previous research on interviewing NNESs (Martin, Evans, \& Baralt, in preparation) as well as theoretical predictions outlined in the 
introduction. Other results revealed significant differences between the samples in terms of English speaking ability and comfort communicating in English, such that the NNES group had lower abilities and was less comfortable than the native speakers group. Taken together, it is clear that communicating in a non-native language posed a significant obstacle for NNESs.

Interestingly, an interaction emerged where the second recall attempt in the control condition — but not the reverse order instruction condition — was particularly helpful for native English speakers but not NNESs. The same interaction pattern was also found (with marginal significance) when analyzing number of details reported across the full interview. The interaction suggests that simply asking (native English speaking) witnesses to think hard and try again is more effective at eliciting details than the reverse order mnemonic. The lack of an effect among NNESs may speak to the difficulty that NNESs face with reporting their memories in a second language. Even though the control interview was less difficult than the reverse order interview overall (according to participants' ratings of interview difficulty), NNESs still did not benefit as expected from the control interview (i.e., there was no difference in amount of new information generated in response to the control and reverse order conditions for NNESs).

It is reassuring, though, there was no significant difference in number of units reported by native English speakers and NNESs in response to the initial free recall question. The comparable rates of productivity indicate that, in best practice investigative interviewing conditions, NNESs are able to provide reasonably complete reports that may assist police with solving crimes. In other words, the additional cognitive load of speaking a second language is not so burdensome that it prohibits them from participating 
in the legal process. While the result is promising, the next step is to identify an interviewing mnemonic that can enhance their participation even more, like the second recall attempt does for native speakers in the current study. Their participation in interviews can help expedite the administration of justice in domestic violence, counterterrorism, and many other types of cases. Considering some police departments neglect to interview NNESs at all (Rivera \& Zraick, 2010), any protocol that encourages NNESs' participation in the justice system is an important step forward.

Accuracy of reports. In the current study, native English speakers provided significantly higher numbers of accurate units than NNESs, but proportions of accurate units (i.e., number of accurate units divided by total number of units reported) were not different between the language groups. The significant difference in number of accurate units, therefore, is driven by the effect of language on productivity (number of units reported). In other words, the accuracy rate is not different between the language groups because, although native English speakers reported more accurate details than NNESs, they also reported more details overall.

Further, NNESs in the control condition provided significantly higher proportions of subjective units compared to native speakers in the reverse order condition. Units were scored subjective when they could not be verified by the mock crime video; for example, units like "sneaking," "in a hurry," and "seemed suspicious" were coded as subjective in the current study. The difference in proportion of subjective units may be explained in several ways. First, it is possible that NNESs opted to provide very coarse-grained details to avoid committing errors of commission. For example, if NNESs were not sure whether the thieves were walking or running (but they knew the thieves were not moving slowly), 
they may have reported "in a hurry" to maintain accuracy in the broadest sense. Second, and relatedly, NNESs may have struggled to find the most precise words in their L2 to express their thoughts. As such, they may have used more less complex syntax to express their memories. Third, it may be normative in other languages and cultures to include more subjective details than verifiable, precise details. That is, it is possible that reporting subjective information — like personal cognitive states ("seemed to me" or "I thought")— is relatively more common in other languages than English (Ikegami, 2005). Whether subjectivity varies by language group could be studied empirically; the current sample is likely underpowered to conduct such analyses given the heterogeneity in language and cultural backgrounds of participants.

There was also a marginally significant interaction of language and interview in the transfer of control condition, such that NNESs reported somewhat higher proportions of repeated details when given the transfer of control instruction than when given only basic, control instructions. A possible explanation is that perhaps the transfer of control instruction resulted in pressure to do "well" on the task, thereby adding additional cognitive load and impairing their metacognitive monitoring skills. The additional pressure to produce details may have caused NNESs to focus on repeating details they were sure of rather than pushing the boundaries of their memory stores.

It is also important to consider that the NNESs in the current study were still reasonably proficient in English. Though they may not have been fluent or fully bilingual, they still rated their proficiency in speaking English, on avergage, at a 5 (out of 7), indicating they spoke English "well.” Therefore, the current results may not generalize to witnesses who are very low proficiency NNESs (e.g., very recent 
immigrants or international tourists). However, the current sample represents the segment of NNESs who would likely be interviewed by police: namely, NNESs proficient enough to communicate in English at a basic level. Police may be more willing to seek interpreters when interviewing witnesses with extremely low proficiency in English; if witnesses are somewhat proficient in English, police may move forward with the interview in English.

\section{Utility of Individual CI Mnemonics}

The secondary hypotheses of the present dissertation relate to the utility of CI mnemonics absent the rest of the CI. In the present study, the reverse order mnemonic and the transfer of control instruction were tested only in conjunction with an initial rapport building session and open-ended question format.

Units of information. Results indicate that the control procedure (i.e., a second recall attempt with no order specified) produced a higher quantity of details compared to the reverse order procedure. Furthermore, the control procedure produced more new information (i.e., more information not previously reported in the first recall attempt) than the reverse order procedure. The finding contributes to the minimal research that isolates CI mnemonics. Previous findings are mixed, and the current study adds to the mixture. In contrast to all of the previous findings, the current study found a benefit of the control second-recall attempt relative to the reverse order instruction in native English speakers. Boon and Noon (1994) found support for the use of isolated CI mnemonics, including a change order instruction, while Milne and Bull (2002) and Memon and colleagues (1996) found no differences in number of details elicited by isolated mnemonics compared to a control. Perhaps the differences among these studies can be 
attributed to variations in how the reverse order (or change order) mnemonic is used in each study. Boon and Noon (1994), for example, asked participants to provide a written statement, whereas the other studies (including the present study) collected verbal reports. Writing and speaking rely on different cognitive procedures; for example, writing allows participants to reflect and revise before submission, whereas speaking does not. Including introductory transfer of control instructions did not elicit significantly more details for either native speakers or NNESs. However, while there was no significant effect, the mean number of details for both language groups were slightly higher in the predicted direction-i.e., in the instructions condition compared to the control condition. It is possible that the effects of the transfer of control instruction, without any other CI mnemonics, are small and that the current study is underpowered to detect such small effects.

Accuracy of reports. Neither of the CI mnemonics produced significant differences in accuracy of reports between language conditions. Although quantity of accurate units was significantly smaller in the reverse order condition than in the control condition, the proportions of accurate units were comparable between interview conditions. Accuracy rates, defined as the number of accurate units divided by the total number of units (or total number of details) given, were also comparable within participants. In other words, the difference in quantity of accurate units was driven by the difference in productivity overall (total number of units reported). Further, both strategies at recall time 2 (i.e., the control instruction and the reverse order instruction) elicited similarly accurate reports. Main effects for quantity and proportion of accurate units were also comparable between the control and transfer of control conditions. The lack of 
differences suggests that - consistent with previous research on the CI (Memon,

Meissner, \& Fraser, 2010)—CI mnemonics produce reports with similar accuracy rates as comparison interviews.

Accuracy of participants' reports was also comparable between the first and second recall attempts in the control and reverse order condition. The lack of differences suggests that participants were still reporting details about which they were reasonably confident, even during the second recall. Participants in the control condition reported more new information than participants in the reverse order condition at the same level of accuracy. It is possible that the reverse order condition is most beneficial when participants have reported all the details about which they are reasonably confident. For example, perhaps the reverse order instruction would be most beneficial when given after multiple "try again" retrieval attempts.

\section{Implications}

Theoretical implications. The results of the current study lend support to two propositions: (1) that speaking a second language is cognitively taxing and (2) that the reverse order mnemonic of the $\mathrm{CI}$ is cognitively taxing.

The main effects of language throughout the study provide support for the first proposition. Though NNESs provided a comparable number of details to native speakers in response to the first interview question, they provided fewer details than native speakers in response to the second. Even without additional, cognitively taxing tasks, NNESs may have been unable to fully report the contents of their memories.

The interaction effects showing a greater benefit (for native speakers) of the control question but not the reverse order question lend support to the second proposition. 
That is, when faced with a cognitively easier task, native speakers excelled; when faced with a more cognitively challenging task, native speakers provided fewer details. The same prediction would be reasonable for NNESs, but that pattern of results did not emerge. Although they are already cognitively loaded (by virtue of using their second language), NNESs are still able to report as many details to the reverse order question as are native speakers. It is possible that cognitive load manifested in some way other than decreasing raw quantity of details. In particular, the main dependent variable affected by increased cognitive load was proportion of subjective details. The increase in proportion of subjective details may indicate that the added cognitive load of the reverse order mnemonic taxes metacognitive monitoring processes rather than retrieval processes. Another component of the current study was a test of the transfer of control instruction. It did not elicit increases in number of details for participants in either language condition. It is possible that the effect is small, and the current study is underpowered to detect it. It is also possible that the transfer of control instruction is particularly useful with less-than-ideal questions. The interview questions in the current study were broad and open-ended. Possibly, the transfer of control instruction and the phrasing of the open-ended question communicated the same message to participants: tell the interviewer as much as possible. If the initial question were phrased differently (e.g., "What did you see?" rather than "Tell me everything you can remember about what you saw"), the transfer of control instruction might have had a more powerful effect. Interestingly, the transfer of control instruction resulted in (marginally significant) increased proportions of repeated details among NNESs. Accuracy rates, however, were unaffected. Overall, reports given by NNESs are reliably accurate, although the reports 
may contain relatively high proportions of repeated details (compared to reports from native speakers).

Practical implications. The results of the present study highlight the importance of providing multiple retrieval attempts to eyewitnesses as well as avoiding cognitively complex interviewing strategies. The native English speakers in the control condition provided the greatest number of details about the mock crime. With native English speakers, the current study suggests that providing a simple "try again" prompt is preferable to a reverse order prompt (if the objective is to maximize number of details provided by the interviewee).

Practitioners should be aware that NNESs are able to provide reasonably detailed narratives in response to open-ended, free recall questions. The mean numbers of details provided by NNESs were comparable between the first and second recall attempts across conditions in the current study. Thus, interviewers should certainly begin interviews with NNESs by asking open-ended free recall questions. In the current study, format of the second question did not elicit significant differences. Therefore, it may not matter whether follow-up questions are framed as "try again" or reverse order prompts. However, it is probably simplest for both interviewers and witnesses/victims to opt for the "try again" (control) recall attempt.

That native English speakers' and NNESs' reports are comparably accurate also has important practical implications. Research shows that observers are more skeptical of statements given by non-native speakers than the same statements given by native speakers (Hanzlíková \& Skarnitzl, 2017; Lev-Ari \& Keysar, 2010). Bias on the basis of accent manifests in legal settings such that observers are likely to rate statements given 
by NNESs as low in credibility (Frumkin, 2004). Interviewers, like police officers or customs officials, may be skeptical of reports given by NNESs. However, the results of the current study show that statements given by NNESs are just as accurate as those given by native English speakers.

Regarding practical implementation of the $\mathrm{CI}$ in the field, the current study implies that investigators may be able to forego lengthy introductory instructions and/or complex retrieval mnemonics in favor of a simple repeated recall session. Abbreviated CIs have been shown to be comparable to full-length CIs in previous research (Dando, Wilcock, Milne, \& Henry, 2009; Memon, Meissner, \& Fraser, 2010). However, it is important to consider the limitations of the current study—namely, that only two specific CI mnemonics were tested in isolation of each other.

\section{Limitations}

Sample characteristics. Levene's test indicated unequal variances between the native speakers and NNES groups. Unequal variances may be caused by unequal group sizes between the two conditions; approximately twice as many native speakers participated in the study than NNES. Unequal variances may have also been caused by differences in populations between native speakers and NNES. For example, native speakers were recruited from the departmental participation pool, whereas NNES were recruited from English programs, community members, and the participant pool. As a result, participants in the NNES group reported a large range of English proficiency data (standard deviation of speaking ability $=1.46$ ); in contrast, native English speakers' ratings of English proficiency were largely homogenous and near the ceiling of the scale (standard deviation of speaking ability $=0.50$ ). 
A large proportion (at least 35.6\% [ $n=23]$ ) of the native speaking sample was bilingual in English and another language. These participants indicated learning English as well as another language in early childhood (e.g., from birth). Because participants differ in how thoroughly they completed the $\mathrm{LHQ}^{3}$, the estimate of $35.6 \%$ being fully bilingual is likely conservative. The cognitive processes involved in describing, for example, actions, directions, and other details may differ between English monolingual and bilingual participants. That is, different languages follow different rules for constructing speech; when speakers have two relatively complete sets of linguistic rules (i.e., a balanced bilingual, who speaks two languages equally well), they may construct speech differently than speakers with only one set of linguistic rules. Work by Filipovic (2011) suggests that bilingual participants may produce descriptions that are the "best of both worlds": they construct speech in a way that would make sense in either language. A concrete example might be participants' descriptions of the perpetrators' actions in the present study. Whereas a monolingual English speaker might say "they rushed out [of the room]," a Spanish-English bilingual participant might say "they left out of the room running." The latter is more consistent with how the phrase would be constructed in Spanish, e.g., "salieron corriendo del cuarto." The latter construction contains more units of information (i.e., "left" [a verb] and "running" [here, an adverb] would be scored as two different units under the current unitization scheme, but "rushed" [a verb] would count as only one unit). Thus, to the extent that NNESs' speech patterns develop in a "best of both worlds" way, perhaps the most appropriate comparison group for novice

\footnotetext{
${ }^{3}$ Some participants reported checkmarks instead of quantitative data, and others included data only for their second languages (e.g., one participant only included proficiency data for American Sign Language).
} 
NNESs would be to balanced bilingual participants. It may also be important to further code the units for accuracy and precision, so that multiple units referring to the same action or object do not artificially inflate group means.

Furthermore, the distribution of educational attainment differs between the language groups. Whereas the vast majority of native English speakers report being enrolled in college (i.e., an undergraduate program), over $20 \%$ of NNES participants reported being enrolled in or having completed graduate school.

Methodological limitations. The current study employed a relatively good control interview protocol. Specifically, research assistants were trained to ask a broad, open-ended free recall question and to not interrupt participants during their recall. Although the free recall question is absent other memory-enhancing mnemonics, free recall is perhaps the most commonly recommended "best practice" investigative interviewing technique. Furthermore, it is not necessarily consistent with real-world witness interviewing techniques. Field studies indicate that investigators often interrupt their witnesses and use complex, multi-part questions (Schreiber Compo, Hyman Gregory, \& Fisher, 2012). Thus, the use of a high-quality control condition may have made it particularly difficult to find significant differences across interview conditions. As such, the results in the current study may underestimate the true effects of these interviewing strategies in ecologically valid scenarios. However, the use of such a control group was important for ensuring that the differences across conditions were well controlled, maximizing internal validly. Further, had low quality interview procedures been used in the control group, the research could be criticized for not implementing a strong test of the hypotheses. 
Another limitation of the interviewing protocols in the current study is that they are a relatively low-stress, low-stakes conversation compared to real police interviews. The power differential between police officer and witness/victim is not present in the current study. Thus, it is likely that NNESs did not face the same social barriers to discussion that real crime witnesses/victims do. The participants in the current study also volunteered to participate, primarily under the guise of an opportunity to practice English. So, the participants in the study were likely more motivated to talk than witnesses/victims who are interviewed by police. Thus, the differences between language conditions on number of details are likely underestimates, possibly obfuscating the potential benefits of the transfer of control instruction.

\section{Future Directions}

The current study is a first step toward recruiting diverse populations in investigative interviewing research. Very few studies in the investigative interviewing literature, if any, have specifically recruited diverse samples. The generalizability of previous research is largely limited to the culture in which the research was conducted (e.g., American samples that exclusively recruit undergraduate student participants). Future research should specifically seek to recruit participant from ethnically, culturally, and linguistically diverse samples. Increasing generalizability on these dimensions is critical for making policy recommendations in a society that is rapidly diversifying.

Future research on eyewitness memory among NNESs should also test other interviewing techniques, from the $\mathrm{CI}$ and otherwise, that facilitate communication. Previous research indicates that having witnesses narrate verbally while they sketch can help elicit more details compared to simply recalling the event (Dando, Wilcock, \& 
Milne, 2008). The sketch is helpful because it allows witnesses to self-generate retrieval cues, lightens the cognitive load of the interview (by providing an external memory aid), and facilitates nonverbal recall. Sketching may be a particularly productive interviewing technique for NNESs.

Other interviewing techniques, such as the timeline method, may also help NNESs communicate effectively. The timeline technique requires witnesses to describe people, actions, and other relevant details on index cards, and then link those items together on a physical timeline (Hope, Mullis, \& Gabbert, 2013). Using a timeline is one way that multiple and varied retrieval could be instantiated for NNESs (first describe the people, then describe the actions, etc.). The timeline may also help alleviate some of the cognitive load experienced by NNESs: allowing them to write their descriptions on cards can free up cognitive resources. Moreover, the process of manipulating physical index cards may assist NNESs with nonverbal output.

It would also be worthwhile to test other interviewing procedures that are productive for native speakers but may be counterproductive for NNESs. The change perspective mnemonic, another aspect of the original CI, may be one of these techniques. The change perspective mnemonic is another cognitively demanding task that, like the reverse order technique, may produce no benefit to NNESs despite being a commonly cited interviewing strategy.

Researchers may also turn their attention to developing a completely novel interviewing strategy that addresses the specific challenges faced by NNESs. Interviewing techniques that minimize cognitive load (e.g., eyeclosure or simplifying questions) and facilitate word retrieval (e.g., gesturing) could make up a cognitive prong 
of a novel interviewing strategy. Social factors, like increasing confidence in a witness's language ability or decreasing state-level anxiety, may be critical components of building rapport with NNESs. Future research could identify the key predictors of detail reporting among NNESs and design an interviewing strategy to maximize reporting.

In sum, it is important to recognize the challenges that NNESs face during investigative interviews as well as the challenges that interviewers face when a language barrier exists between the interviewer and witness/victim. The next step is to work toward developing an interview strategy that is sensitive to these challenges, so that NNESs can have equitable access, representation, and justice within the legal system. 


\section{REFERENCES}

Anderson, R. C., \& Pichert, J. W. (1978). Recall of previously unrecallable information following a shift in perspective. Journal of Verbal Learning and Verbal Behavior, 17, 1-12. doi:10.1016/S0022-5371(78)90485-1

Ardila, A. (2003). Language representation and working memory with bilinguals. Journal of Communication Disorders, 36, 233-240.

Ballard, P. B. (1913). Oblivescence and reminiscence. British Journal of Psychology Monograph Supplements, 1, 1-82.

Bellezza, F. S., \& Bower, G. H. (1982). Remembering script-based texts. Poetics, 11, 123. doi:10.1016/0304-422X(82)90002-X

Boon, J., \& Noon, E. (1994). Changing perspectives in cognitive interviewing. Psychology, Crime \& Law, 1, 59-69. doi:10.1080/10683169408411936

Bornstein, B. H., Liebel, L. M., \& Scarberry, N. C. (1998). Repeated testing in eyewitness memory: A means to improve recall of a negative emotional event. Applied Cognitive Psychology, 12, 119-131. doi:10.1002/(SICI)10990720(199804)12:2<119::AID-ACP500>3.0.CO;2-4

Camarota, S. A., \& Zeigler, K. (2014, October 3). One in five U.S. resident speak foreign language at home, record 61.8 million. Retrieved from Center for Immigration Studies: https://cis.org/One-Five-US-Residents-Speaks-Foreign-Language-HomeRecord-618-million

Celce-Murcia, M., Dörnyei, Z., \& Thurrell, S. (1995). Communicative Competence: A Pedagogically Motivated Model with Content Specifications. Issues in Applied Linguistics, 6, 5-35.

Cicchetti, D. V. (1994). Guidelines, criteria, and rules of thumb for evaluating normed and standardized assessment instruments in psychology. Psychological Assessment, 6, 284-290. doi:10.1037/1040-3590.6.4.284

Clément, R. (1980). Ethnicity, contact and communicative competence in a second language. In H. Giles, W. P. Robinson, \& P. M. Smith (Eds.), Social Psychology and Language (pp. 147-159). Oxford: Pergamon.

Collins, R., Lincoln, R., \& Frank, M. G. (2002). The effect of rapport in forensic interviewing. Psychiatry, Psychology and Law, 9, 69-78.

doi:10.1375/pplt.2002.9.1.69

Dando, C., Wilcock, R., \& Milne, R. (2008). The cognitive interview: Inexperienced police officers' perceptions of their witness/victim interviewing practice. Legal and Criminological Psychology, 13, 59-70. doi:10.1348/135532506X162498 
Dando, C., Wilcock, R., Milne, R., \& Henry, L. (2009). A modified cognitive interview procedure for frontline police investigators. Applied Cognitive Psychology, 23, 698-716. doi:10.1002/acp.1501

Eugenio, P., Buckhout, R., Kostes, S., \& Ellison, K. W. (1982). Hypermnesia in the eyewitness to a crime. Bulletin of the Psychonomic Society, 19, 83-86. doi:10.3758/BF03330047

Ewens, S., Vrij, A., Leal, S., Mann, S., Jo, E., \& Fisher, R. P. (2016a). The effect of interpreters on eliciting information, cues to deceit and rapport. Legal and Criminological Psychology, 21, 286-304. doi:10.1111/lcrp.12067

Ewens, S., Vrij, A., Leal, S., Mann, S., Jo, E., Shaboltas, A., . . Houston, K. (2016b). Using the model statement to elicit information and cues to deceit from native speakers, non-native speakers, and those talking through an interpreter. Applied Cognitive Psychology, 30, 854-862. doi:10.1002/acp.3270

Ewens, S., Vrij, A., Mann, S., \& Leal, S. (2016c). Using the reverse order technique with non-native speakers or through an interpreter. Applied Cognitive Psychology, 30, 242-249. doi:10.1002/acp.3196

Filipović, L. (2011). Speaking and remembering in one or two languages: bilingual vs. monolingual lexicalization and memory for motion events. International Journal of Bilingualism, 15, 466-485. doi:10.1177/1367006911403062

Fisher, R. P., \& Geiselman, R. E. (1992). Memory-enhancing techniques for investigative interviewing: The cognitive interview. Springfield, IL: Charles C. Thomas, Publisher.

Fisher, R. P., \& Geiselman, R. E. (2010). The Cognitive Interview method of conducting police interviews: eliciting extensive information and promoting therapeutic jurisprudence. International Journal of Law and Psychiatry, 33, 321-328. doi:10.1016/j.ijlp.2010.09.004

Fisher, R. P., Milne, R., \& Bull, R. (2011). Interviewing cooperative witnesses. Current Directions in Psychological Science, 20, 16-19. doi:10.1177/0963721410396826

Frumkin, L. (2004). Influences of accent and ethnic background on perceptions of eyewitness testimony. Psychology, Crime \& Law, 13, 317-331. doi:10.1080/10683160600822246

Geiselman, R. E., \& Callot, R. (1990). Reverse versus forward recall of script-based texts. Applied Cognitive Psychology, 4, 141-144. doi:10.1002/acp.2350040206

Geiselman, R. E., \& Fisher, R. P. (2014). Interviewing witnesses and victims. In M. St. Yves (Ed.), Investigative Interviewing: Handbook of Best Practices (pp. 29-40). Toronto: Thomson Reuters Publishers. 
Geiselman, R. E., Fisher, R. R., MacKinnon, D. P., \& Holland, H. L. (1986). Enhancement of eyewitness memory with the cognitive interview. The American Journal of Psychology, 99, 385-401. doi:10.2307/1422492

Gilbert, J. A., \& Fisher, R. P. (2006). The Effects of Varied Retrieval Cues on Reminiscence in Eyewitness Memory. Applied Cognitive Psychology, 20, 723739. doi:10.1002/acp.1232

Godden, D. R., \& Baddeley, A. D. (1975). Context-dependent memory in two natural environments: On land and underwater. British Journal of Psychology, 66, 325331. doi:10.1111/j.2044-8295.1975.tb01468.x

Hanzlíková, D., \& Skarnitzl, R. (2017). Credibility of native and non-native speakers of English revisited: Do non-native listeners feel the same? Research in Language, 15, 285-298. doi:10.1515/rela-2017-0016

Hatch, E. (1992). Discourse and language education. Cambridge: Cambridge University Press.

Hope, L., Mullis, R., \& Gabbert, F. (2013). Who? What? When? Using a timeline technique to facilitate recall of a complex event. Journal of Applied Research in Memory and Cognition, 2, 20-24. doi:10.1016/j.jarmac.2013.01.002

Hope, L., Mullis, R., \& Gabbert, F. (2013). Who? What? When? Using a timeline technique to facilitate recall of a complex event. Journal of Applied Research in Memory and Cognition, 2, 20-24. doi:10.1016/j.jarmac.2013.01.002

Ikegami, Y. (2005). Indices of a 'subjectivity-prominent' language: Between cognitive linguistics and linguistic typology. Annual Review of Cognitive Linguistics, 3, 132-164. doi:10.1075/arcl.3.08ike

Kapatsinksi, V. (2010). Frequency of use leads to automaticity of production: Evidence from repair in conversation. Language and Speech, 53, 71-105. doi:10.1177/0023830909351220

Köhnken, G., Milne, R., Memon, A., \& Bull, R. (1999). The cognitive interview: A metaanalysis. Psychology, Crime \& Law, 5, 3-27. doi:10.1080/10683169908414991

Koo, T. K., \& Li, M. Y. (2016). A Guideline of Selecting and Reporting Intraclass Correlation Coefficients for Reliability Research. Journal of Chiropractic Medicine, 15, 155-163. doi:10.1016/j.jcm.2016.02.012

Kroll, J. F., \& Curley, J. (1988). Lexical memory in novice bilinguals: The role of concepts in retrieving second language words. In M. Gruneberg, P. Morris, \& R. Sykes (Eds.), Practical Aspects of Memory (Vol. 2, pp. 389-395). London: John Wiley \& Sons. 
Leibowitz, H. W., Guzy, L. T., Peterson, E., \& Blake, P. T. (1993). Quantitative perceptual estimates: Verbal versus nonverbal retrieval techniques. Perception, 22, 1051-1060. doi:10.1068/p221051

Lev-Ari, S., \& Keysar, B. (2010). Why don't we believe non-native speakers? The influence of accent on credibility. Journal of Experimental Social Psychology, 46, 1093-1096. doi:10.1016/j.jesp.2010.05.025

Levelt, W. J. (1989). Speaking: From intention to articulation. Cambridge, Massachusetts: MIT Press.

Levelt, W. J., Roelofs, A., \& Meyer, A. S. (1999). A theory of lexical access in speech production. Behavioral and Brain Sciences, 22, 1-38. doi:10.1017/S0140525X99001776

Li, P., Sepanski, S., \& Zhao, X. (2006). Language history questionnaire: A web-based interface for bilingual research. Behavior Research Methods, 38, 202-210. doi:10.3758/BF03192770

Li, P., Zhang, F., Yu, A., \& Zhao, X. (2019). Language History Questionnaire (LHQ3): An enhanced tool for assessing multilingual experience. Bilingualism: Language and Cognition, 1-7. doi:10.1017/S1366728918001153

Loftus, E. F. (2005). Planting misinformation in the human mind: A 30-year investigation of the malleability of memory. Learning \& Memory, 12, 361-366. doi:10.1101/lm.94705

MacIntyre, P. D., Dörnyei, Z., Clément, R., \& Noels, K. A. (1998). Conceptualizing willingness to communicate in a L2: A situational sodel of L2 confidence and affiliation. The Modern Language Journal, 82, 545-562. doi:10.1111/j.15404781.1998.tb05543.x

Martin, J. A., Evans, J. R., \& Baralt, M. (in preparation). Variations in witness memory reports as a function of language proficiency.

Memon, A., \& Higham, P. A. (1999). A review of the cognitive interview. Psychology, Crime and Law, 5, 177-196. doi:10.1080/10683169908415000

Memon, A., Cronin, O., Eaves, R., \& Bull, R. (1996). An empirical test of the 'mnemonic components' of the cognitive interview. In G. M. Davies, S. Lloyd-Bostock, M. McMurran, \& C. Wilson (Eds.), Psychology and Law: Advances in Research. Berlin: de Gruyter.

Memon, A., Meissner, C., \& Fraser, J. (2010). The cognitive interview: A meta-analytic review and study space analysis of the past 25 years. Psychology, Public Policy, and Law, 16, 340-372. doi:10.1037/a0020518 
Menenti, L., \& Indefrey, P. (2006). L2-L1 word association in bilinguals: Direct evidence. Nijmegen CNS, 1, 17-24.

Milne, R., \& Bull, R. (2002). Back to basics: A componential analysis of the original cognitive interview mnemonics with three age groups. Applied Cognitive Psychology, 16, 743-753. doi:10.1002/acp.825

Morris, C. D., Bransford, J. D., \& Franks, J. J. (1977). Levels of processing versus transfer appropriate processing. Journal of Verbal Learning and Verbal Behavior, 16, 519-533. doi:10.1016/S0022-5371(77)80016-9

Otgaar, H., van Ansem, R., \& Pauw, C. H. (2016). Improving children's interviewing methods? The effects of drawing and practice on children's memories for an event. Journal of Police and Criminal Psychology, 31, 279-287. doi:10.1007/s11896-016-9190-0

Paivio, A., \& Desrochers, A. (1980). A dual-coding approach to bilingual memory. Canadian Journal of Psychology/Revue canadienne de psychologie, 34, 388-399. doi:10.1037/h0081101

Paulo, R. M., Albuquerque, P. B., \& Bull, R. (2013). The enhanced cognitive interview: Towards a better use and understanding of this procedure. International Journal of Police Science \& Management, 190-199. doi:10.1350/ijps.2013.15.3.311

Payne, D. G. (1987). Hypermnesia and reminiscence in recall: A historical and empirical review. Psychological Bulletin, 101(1), 5-27. doi:10.1037/0033-2909.101.1.5

Potter, M. C., So, K.-F., Von Eckardt, B., \& Feldman, L. B. (1984). Lexical and conceptual representation in beginning and proficient bilinguals. Journal of Verbal Learning and Verbal Behavior, 23, 23-38. doi:10.1016/S00225371(84)90489-4

Rivera, R., \& Zraick, K. (2010, November 18). Audit says police fall short in providing interpreters. The New York Times, p. A14. Retrieved from https://www.nytimes.com/2010/11/19/nyregion/19translate.html

Roediger, H. L., \& Payne, D. G. (1982). Hypermnesia: The role of repeated testing. Journal of Experimental Psychology: Learning, Memory, and Cognition, 8, 6672. doi:10.1037/0278-7393.8.1.66

Schreiber Compo, N., Hyman Gregory, A., \& Fisher, R. (2012). Interviewing behaviors in police investigators: a field study of a current US sample. Psychology, Crime \& Law, 18, 359-375. doi:10.1080/1068316X.2010.494604

Shaffer, S. A., \& Evans, J. R. (2018). Interpreters in law enforcement contexts: Practices and experiences according to investigators. Applied Cognitive Psychology, 32, 150-162. doi:10.1002/acp.3388 
Shaw, J. S., Garcia, L. A., \& Robles, B. E. (1997). Cross-language postevent misinformation effects in Spanish-English bilingual witnesses. Journal of Applied Psychology, 82, 889-899. doi:10.1037/0021-9010.82.6.889

Skehan, P. (2001). Task and language performance assessment. In M. Bygate, P. Skehan, \& M. Swain (Eds.), Researching pedagogic tasks: Second language learning, teaching, and testing (pp. 167-187). Harlow, UK: Pearson Education.

Skehan, P. (2009). Modelling second language performance: Integrating complexity, accuracy, fluency, and lexis. Applied Linguistics, 30, 510-532. doi:10.1093/applin/amp047

Tulving, E., \& Thomson, D. M. (1973). Encoding specificity and retrieval processes in episodic memory. Psychological Review, 80, 352-373. doi:10.1037/h0020071

Turtle, J. W., \& Yuille, J. C. (1994). Lost but not forgotten details: Repeated eyewitness recall leads to reminiscence but not hypermnesia. Journal of Applied Psychology, 79, 260-271. doi:10.1037/0021-9010.79.2.260

Vallano, J. P., \& Schreiber Compo, N. (2015). Rapport-building with cooperative witnesses and criminal suspects: A theoretical and empirical review. Psychology, Public Policy, and Law, 21, 85-99. doi:10.1037/law0000035

Vredeveldt, A., \& Penrod, S. D. (2013). Eye-closure improves memory for a witnessed event under naturalistic conditions. Psychology, Crime \& Law , 19, 893-905. doi:10.1080/1068316X.2012.700313

Vredeveldt, A., Baddeley, A. D., \& Hitch, G. J. (2013). The effectiveness of eye-closure in repeated interviews. Legal and Criminological Psychology, 19, 282-295. doi:10.1111/lcrp. 12013

Vrij, A., Leal, S., Mann, S., \& Fisher, R. (2012). Imposing cognitive load to elicit cues to deceit: Inducing the reverse order technique naturally. Psychology, Crime \& Law, 18, 579-594. doi:10.1080/1068316X.2010.515987

Wakefield, S. J., Kebbell, M. R., Moston, S., \& Westera, N. (2015). Perceptions and profiles of interviews with interpreters: A police survey. Australian \& New Zealand Journal of Criminology, 48, 53-72. doi:10.1177/0004865814524583 


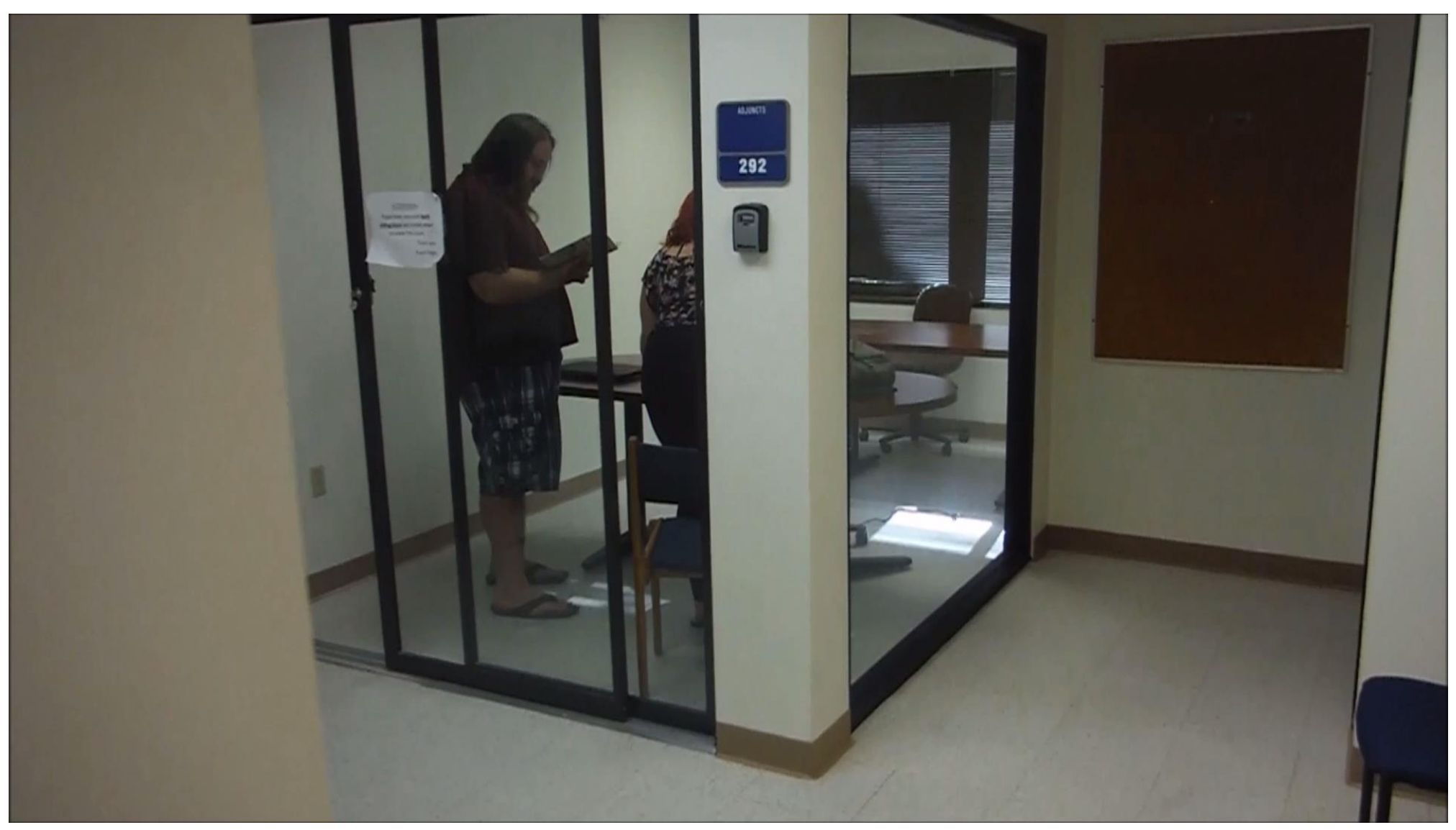

Figure 13. Crime scene from stimulus video. 


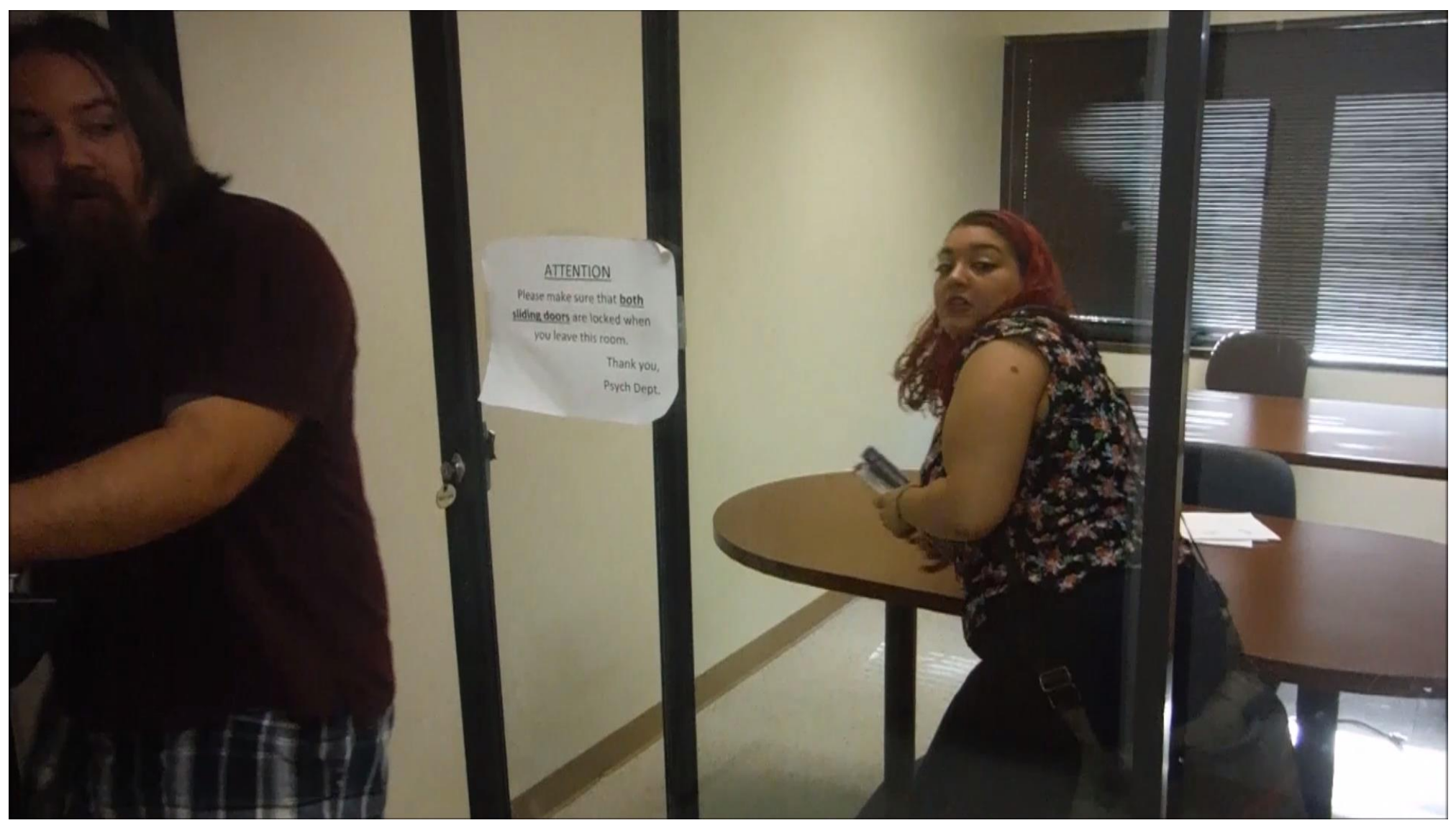

Figure 14. Perpetrators from stimulus video 
APPENDICES 
Appendix A

\section{L2 Language History Questionnaire (Version 3.0, 2015) See http://blclab.org/ for online use and credit}

Participant ID:

1. Age (in years):

2. Sex (Circle one): Male / Female

3. Education (your current or most recent educational level, even you have not finished the degree) (Circle one):

- Graduate school (PhD/MD/JD) • High school

- Graduate school (Masters) - Middle school

- College (BA/BS) - Other (specify):

4. Have you ever studied or learned a second language in terms of listening, speaking, reading, or writing? (Circle one):

Yes / No

5. Indicate your native language(s) and any other languages you have studied or learned, the age at which you started using each language in terms of listening, speaking, reading, and writing, and the total number of years you have spent using each language.

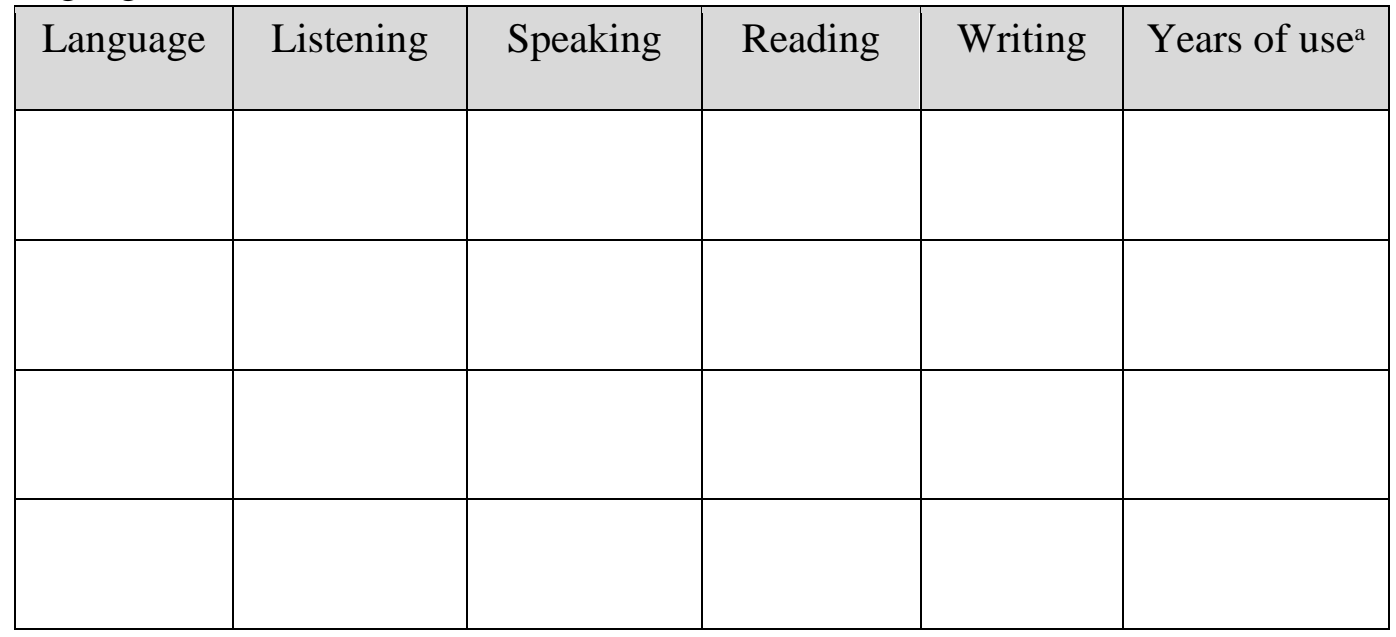

a. $\quad$ You may have learned a language, stopped using it, and then started using it again. Please give the total number of years. 
6a. Country of residence:

6b. Country of origin:

$6 c$. If $6 a$ and $6 b$ are different, then when did you first move to the country where you currently live?

7. If you have lived or travelled in countries other than your country of residence or country of origin for three or more months, then indicate the name of the country, your length of stay, the language you used, and the frequency of your use of the language for each country.

\begin{tabular}{|l|l|l|l|}
\hline Country & $\begin{array}{c}\text { Length of stay } \\
{[\text { month(s) }}\end{array}$ & Language & $\begin{array}{c}\text { Frequency of } \\
\text { use }^{\mathrm{b}}\end{array}$ \\
\hline & & & 1234567 \\
\hline & & & 1234567 \\
\hline & & & 1234567 \\
\hline
\end{tabular}

a. You may have been to the country on multiple occasions, each for a different length of time. Add all the trips together.

b. Please rate according to the following scale (circle the number in the table)

\begin{tabular}{cccccccc}
\hline \multirow{2}{*}{ Never } & Rarely & Sometimes & Regularly & Often & Usually & Always \\
1 & 2 & 3 & 4 & 5 & 6 & 7 \\
\hline
\end{tabular}


8. Indicate the age at which you started using each of the languages you have studied or learned in the following environments.

\begin{tabular}{|l|l|l|c|c|c|c|}
\hline Language & $\begin{array}{l}\text { At } \\
\text { home }\end{array}$ & $\begin{array}{c}\text { With } \\
\text { friends }\end{array}$ & $\begin{array}{c}\text { At } \\
\text { school }\end{array}$ & $\begin{array}{c}\text { At } \\
\text { work }\end{array}$ & $\begin{array}{c}\text { Language } \\
\text { software }\end{array}$ & $\begin{array}{c}\text { Online } \\
\text { games }\end{array}$ \\
\hline & & & & & & \\
\hline & & & & & & \\
\hline & & & & & & \\
\hline & & & & & & \\
\hline
\end{tabular}

9. Indicate the language used by your teachers for instruction at each educational level. If the instructional language switched during any educational level, then also indicate the "Switched to" language.

\begin{tabular}{|c|l|l|}
\hline & Language & (Switched to) \\
\hline Elementary school & & \\
\hline Middle school & & \\
\hline High school & & \\
\hline College/university & & \\
\hline
\end{tabular}


10. Rate your language learning skill. In other words, how good do you feel you are at learning new languages, relative to your friends or other people you know? (circle one)

\begin{tabular}{ccccccc} 
Very poor & Poor & Limited & Average & Good & Very good & excellent \\
\hline 1 & 2 & 3 & 4 & 5 & 6 & 7
\end{tabular}

11. Rate your current ability in terms of listening, speaking, reading, and writing in each of the languages you have studied or learned. Please rate according to the following scale (circle the number in the table):

\begin{tabular}{ccccccc}
\hline Very poor & Poor & Limited & Functional & Good & Very good & Native-like \\
1 & 2 & 3 & 4 & 5 & 6 & 7 \\
\hline
\end{tabular}

\begin{tabular}{|c|c|c|c|c|}
\hline Language & Listening & Speaking & Reading & Writing \\
\hline & 1234567 & 1234567 & 1234567 & 1234567 \\
\hline & 1234567 & 1234567 & 1234567 & 1234567 \\
\hline & 1234567 & 1234567 & 1234567 & 1234567 \\
\hline & 1234567 & 1234567 & 1234567 & 1234567 \\
\hline
\end{tabular}


12. If you have taken any standardized language proficiency tests (e.g., TOEFL), then indicate the name of the test, the language assessed, and the score you received for each.

If you do not remember the exact score, then indicate an "Approximate score" instead.

\begin{tabular}{|l|l|l|l|}
\hline Test & Language & Score & $\begin{array}{c}\text { (Approximate } \\
\text { score) }\end{array}$ \\
\hline & & & \\
\hline & & & \\
\hline & & & \\
\hline & & & \\
\hline
\end{tabular}

13. Rate the strength of your foreign accent for each of the languages you have studied or learned. Please rate the strength of your accent according to the following scale (circle the number in the table):

\begin{tabular}{ccccccc}
\hline None & Very weak & Weak & Moderate & Strong & Very strong & Extreme \\
1 & 2 & 3 & 4 & 5 & 6 & 7 \\
\hline
\end{tabular}

\begin{tabular}{|l|lllllll|}
\hline Language & \multicolumn{1}{|c|}{ Strength of accent } \\
\hline & 1 & 2 & 3 & 4 & 5 & 6 & 7 \\
\hline & 1 & 2 & 3 & 4 & 5 & 6 & 7 \\
\hline & 1 & 2 & 3 & 4 & 5 & 6 & 7 \\
\hline & 1 & 2 & 3 & 4 & 5 & 6 & 7 \\
\hline
\end{tabular}


14. Estimate how many hours per day you spend engaged in the following activities in each of the languages you have studied or learned.

$$
\text { Language: Language: Language: }
$$

Watching television:

Listening to radio:

Reading for fun:

Reading for school/work:

Writing emails to friends:

Writing for school/work: (hrs)

(hrs)

(hrs)

(hrs)

(hrs)

(hrs) (hrs)

(hrs)

(hrs)

(hrs)

(hrs)

(hrs) (hrs)

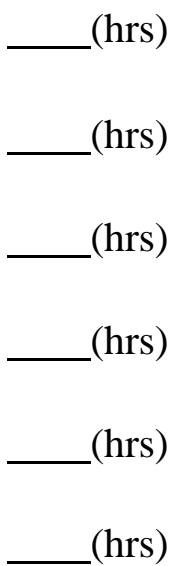

15. Estimate how many hours per day you spend speaking with the following groups of people in each of the languages you have studied or learned.

Language: Language: Language:

Family members:

(hrs)

Friendsa:

(hrs)

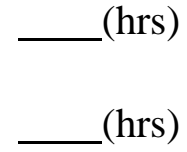

Classmates:

(hrs)

Coworkers ${ }^{\mathrm{b}}$ :

(hrs) (hrs)

(hrs)

(hrs) (hrs)

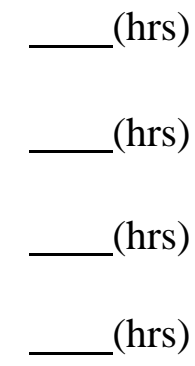

a. Include significant others in this category if you did not include them as family members (e.g., married partners).

b. Include anyone in the work environment in this category (e.g., if you are a teacher, include students as co-workers). 
16a. Do you mix words or sentences from different languages when you speak? (This includes, for example, starting a sentence in one language but using a word or phrase from another language in the middle of the sentence.) (Circle one)

\section{Yes / No}

16b. If you answered "Yes" to 16a, then indicate the languages that you mix and estimate the frequency of mixing in normal conversation with the following groups of people. Please estimate the frequency of mixing according to the following scale (circle the number in the table):

\begin{tabular}{|c|l|l|l|}
\hline & $\begin{array}{c}\text { Language } \\
1\end{array}$ & $\begin{array}{c}\text { Language } \\
2\end{array}$ & $\begin{array}{c}\text { Frequency of } \\
\text { mixing }\end{array}$ \\
\hline Family & & & 1234567 \\
\hline Friends & & & 1234567 \\
\hline Classmates & & & 1234567 \\
\hline Coworkers & & & 1234567 \\
\hline
\end{tabular}

17. In which language do you communicate best or feel most comfortable in terms of listening, speaking, reading, and writing in each of the following environments?

\begin{tabular}{|l|l|l|l|l|}
\hline & Listening & Speaking & Reading & Writing \\
\hline At home & & & & \\
\hline
\end{tabular}




\begin{tabular}{|c|l|l|l|l|}
\hline With & & & & \\
friends & & & & \\
\hline At & & & & \\
school & & & & \\
\hline At work & & & & \\
\hline
\end{tabular}

18. How often do you use each of the languages you have studied or learned for the following activities? Please circle the number in the table according to the scale below.

\begin{tabular}{ccccccc}
\hline Never & Rarely & Sometimes & Regularly & Often & Usually & Always \\
1 & 2 & 3 & 4 & 5 & 6 & 7 \\
\hline
\end{tabular}

\begin{tabular}{|c|c|c|c|c|c|c|}
\hline Language & Thinking & $\begin{array}{c}\text { Talking to } \\
\text { yourself }\end{array}$ & $\begin{array}{l}\text { Expression } \\
\text { emotion }^{\mathrm{a}}\end{array}$ & Dreaming & Arithmetic $^{\mathrm{b}}$ & $\begin{array}{c}\text { Remembering } \\
\text { numbers }\end{array}$ \\
\hline & 1234567 & 1234567 & 1234567 & 1234567 & 1234567 & 1234567 \\
\hline & 1234567 & 1234567 & 1234567 & 1234567 & 1234567 & 1234567 \\
\hline & 1234567 & 1234567 & 1234567 & 1234567 & 1234567 & 1234567 \\
\hline & 1234567 & 1234567 & 1234567 & 1234567 & 1234567 & 1234567 \\
\hline
\end{tabular}

a. This includes shouting, cursing, showing affection, etc.

b. This includes counting, calculating tips, etc.

c. This includes telephone numbers, ID numbers, etc.

19. What percentage of your friends speaks each of the languages you have studied or learned? (The total percentage should add up to 100\%.) 


\begin{tabular}{|r|r|}
\hline Language & Percentage \\
\hline & $\%$ \\
\hline & $\%$ \\
\hline & $\%$ \\
\hline & $\%$ \\
\hline
\end{tabular}

20a. Do you feel that you are bicultural or multicultural? (This includes, for example, growing up with parents or relatives from different cultures or living in different cultures for extensive periods of time.) (Circle one)

Yes / No

20b. If you answered "Yes" to 20a, then which cultures/languages do you identify with more strongly? Rate the strength of your connection in the following categories for each culture/language. Circle the number in the table according to the following scale.

\begin{tabular}{ccccccc}
\hline None & Very weak & Weak & Moderate & Strong & Very strong & Extreme \\
1 & 2 & 3 & 4 & 5 & 6 & 7 \\
\hline
\end{tabular}

\begin{tabular}{|c|c|c|c|c|c|c|}
\hline Culture/Language & $\begin{array}{c}\text { Way of } \\
\text { life }\end{array}$ & Food & Music & Art & $\begin{array}{c}\text { Cities/ } \\
\text { towns }\end{array}$ & $\begin{array}{c}\text { Sports } \\
\text { teams }\end{array}$ \\
\hline & 1234567 & 1234567 & 1234567 & 1234567 & 1234567 & 1234567 \\
\hline & 1234567 & 1234567 & 1234567 & 1234567 & 1234567 & 1234567 \\
\hline & 1234567 & 1234567 & 1234567 & 1234567 & 1234567 & 1234567 \\
\hline & 1234567 & 1234567 & 1234567 & 1234567 & 1234567 & 1234567 \\
\hline
\end{tabular}


21. Please comment below to indicate any additional answers to any of the questions above that you feel better describe your language background or usage.

22. Please comment below to provide any other information about your language background or usage. 
Appendix B

\section{Language \& Interviewing: SONA Protocol}

Don't skim. All the steps are here for you. If you skip a step, it messes up the study.

Setting up

$\checkmark$ Check the tablet to make sure it's working correctly (e.g., internet)

$\checkmark$ Make sure there you have copies of:

Informed consent documents (English)

- Language history questionnaire

- Tablet and audio recorder

- Blank paper to make notes

$\checkmark$ Confirm the conditions based on the running log

- Fill in page 1 of Qualtrics before the study, if you have time

$\checkmark$ Open video; make sure it is ready to play right away

\section{STUDY PROTOCOL}

1. Confirm the participant is at the correct study for the correct time slot. i.e., double-check the Sona email. Ask for their name and/or if they are here for the "Language \& Interviewing" study. Let the participant into the testing room.

2. Give the participant the consent form. Tell the participant:

This is a study researching how language influences the way people respond to different types of questions. Please read through this consent form. It basically tells you that your responses today are anonymous, that the session will be audio recorded, and that you can leave whenever you want to, without penalty. Let me know of any questions you have.

When they finish: Okay, do you have any questions before we begin?

If yes, answer the question. If you cannot answer the question: Sorry, but I can't answer that right now. I can let you know more information at the end of the study, or you can contact the researchers listed on the consent form.

If there are no questions, ask participant to sign AND you must sign. File away.

3. Tell the participant:

I am going to show you a short video. Please pay attention to the video because I'm going to ask you some questions about it later.

4. Play the video. Make sure that the video plays in full screen. 
5. After the video, provide the participant with the Language History Questionnaire. NOTE: Fill in ID number from running log on LHQ! Do NOT let participant fill in Panther ID.

Now, please fill out this questionnaire before we move on. Take as much time as you need. If you have any questions, let me know. If you are uncomfortable responding to any of the questions, just leave it blank.

While the participant is working on this task, confirm the interview condition. Make sure you have that protocol ready.

BEGIN RECORDING NOW. Press record and say: SONA Participant \#

6. GO TO THE INTERVIEW PROTOCOLS!! Follow the protocol for assigned condition. The interview should have six phases:

- Introductory phase

- Instructions (differs across conditions)

- First free recall

- Second recall attempt (differs across conditions)

- Last question

- Conclusion

END RECORDING. Press “stop” on recorder. 
7. Open Qualtrics.

Okay, thank you. Now I have some additional follow-up questions for you to answer. Please fill in your responses on this form.

File away any papers/forms while the participant works on the questionnaire.

8. When the participant finishes the questionnaire, debrief and dismiss.

Thank you for participating today. During the study, you watched a video of a mock crime. It was not a real crime. Then, you provided some details about which languages you speak. Then, you were interviewed about the mock crime you saw. Different participants receive different instructions and different interview questions. We are testing whether native and non-native English speakers respond differently when interviewed with these different techniques. Do you have any additional questions for me?

Answer any questions that you can. If participants ask for any follow-up information (or questions you don't know the answer to), refer them to my email address.

If no questions (or after questions), you can dismiss them:

Okay, you will receive your Sona credit within 24 hours. Thanks/have a good day!

FILING DOCUMENTS: Anything with participant information must be stored in DM 268A in the correct folder. Please file these as soon as you are finished running participants. 
Appendix C

\section{Language \& Interviewing: ELI Protocol}

Don't skim. All the steps are here for you. If you skip a step, it messes up the study.

Setting up

$\checkmark$ Check the tablet to make sure it's working correctly (e.g., internet)

$\checkmark$ Make sure there you have copies of:

$\circ$ Informed consent documents - in all languages

$\circ$ Language history questionnaire

- Tablet and audio recorder

- Blank paper to make notes

$\checkmark$ Confirm the conditions based on the running log

$\circ$ Fill in page 1 of Qualtrics before the study, if you have time

$\checkmark$ Open video; make sure it is ready to play right away

$\checkmark$ Get participant's ELI level (ask participant; should be 1-6; can do at the beginning or during "rapport" interview phase)

Keep in mind:

- You may communicate in another language to clarify consent or debriefing issues (e.g., questions about the study, if there are any penalties or compensation). Start with English, but if the participant has a hard time understanding, you can use another language.

- The interview (step 6) should occur completely in English.

- If you do not speak the participant's native language, rephrase in English as simply as possible. If you still require assistance, contact me and the ELI.

- IF YOU NEED A ROOM: Check for empty classrooms on 1st floor of ELI. If none, ask secretary (2nd floor) or front desk to use a conference room or meeting room.

I (Keith) have emailed with the director, Mr. Sanchez. He has given us permission to recruit participants from the ELI and to use empty rooms if they are available.

\section{STUDY PROTOCOL}

1. Confirm you have the correct participant. (I will cc you on an email in advance.)

2. Give the participant the consent form. Given them a copy in BOTH English and their native Language. Tell the participant:

This is a study researching how language influences the way people respond to different types of questions. Please read through this consent form. It basically tells you that your responses today are anonymous, that the session 
will be audio recorded, and that you can leave whenever you want to, without penalty. Let me know of any questions you have.

When they finish: Okay, do you have any questions before we begin?

If yes, answer the question. If you cannot answer the question: Sorry, but I can't answer that right now. I can let you know more information at the end of the study, or you can contact the researchers listed on the consent form.

If there are no questions, ask participant to sign AND you must sign. Then, file the consent form.

3. Tell the participant:

I am going to show you a short video. Please pay attention to the video because I'm going to ask you some questions about it later.

4. Play the video. Make sure that the video plays in full screen.

5. After the video, provide the participant with the Language History Questionnaire. NOTE: On LHQ, ID = participant ID from running log. Fill this in before you give the LHQ.

Now, please fill out this questionnaire before we move on. Take as much time as you need. If you have any questions, let me know. If you are uncomfortable responding to any of the questions, just leave it blank.

While the participant is working on this task, confirm the interview condition. Make sure you have that protocol ready.

BEGIN RECORDING NOW. Press record and say: ELI Participant \#

6. GO TO THE INTERVIEW PROTOCOLS!! Follow the protocol for assigned condition.

The interview should have six phases:

- Introductory phase

- Instructions (differs across conditions)

- First free recall

- Second recall attempt (differs across conditions)

- Last question

- Conclusion

Do not give instructions to participants in any other language:

Sorry, but I can only give these instructions in English. 
If the participant asks to speak in another language, ask them to use English the best they can:

Please use English the best you can. It's okay if it isn't perfect. I can understand you.

END RECORDING. Press stop on recorder. (Do not press record again - that will drain the battery.)

7. Open Qualtrics.

Okay, thank you. Now I have some additional follow-up questions for you to answer. Please fill in your responses on this form.

File away any papers/forms while the participant works on the questionnaire.

8. When the participant finishes the questionnaire, debrief and dismiss.

Thank you for participating today. During the study, you watched a video of a mock crime. It was not a real crime. Then, you provided some details about which languages you speak. Then, you were interviewed about the mock crime you saw. Different participants receive different instructions and different interview questions. We are testing whether native and non-native English speakers respond differently when interviewed with these different techniques. Do you have any additional questions for me?

Answer any questions that you can. If participants ask for any follow-up information (or questions you don't know the answer to), refer them to my email address.

If no questions (or after questions), you can dismiss them.

NOTE: Give participants a blank copy of the consent form before they leave! 


\section{Appendix D}

\section{Control Interview Protocol}

Consent: Provide participant with informed consent. Say, "This basically tells you that your responses today are anonymous, that the session will be audio recorded, and that you can leave whenever you want to, without penalty. Please read and sign."

Introductory Phase: Introduce yourself. Something like: "Hi, my name is Keith. I'm a researcher with the psychology department. How are you?"

Develop rapport. Spend a couple of minutes talking with the participant. Draw some type of connection with yourself and the participant to make them feel comfortable speaking with you. For example:

"Did you find parking okay?"

"Do you live close to campus?"

"How is your week going?"

"That sounds [like a lot of work] [like a lot of fun] [like an interesting assignment]."

"Do you like that class? I took a similar class and really enjoyed it."

(Any appropriate response to what the participant says)

$\underline{\text { Instructions }}$ Make sure to begin recording at this time.

"You watched a video a few minutes ago. That was a recording of someone committing a crime. What I'm going to do now is ask you some questions about what you saw. Okay, are you ready to begin?"

First free recall: "Please tell me everything you can remember about what you saw." Note: during this time, do not interrupt the participant! Take thorough notes.

Second free recall: "Okay, now I want you to really think about everything you saw. [pause] Please tell me, again, everything that you remember about what you saw."

Last question: "Okay, is there anything else you can tell me about what you saw?" If the participant reports anything else, keep repeating the question.

Repeat until participant responds no.

Conclusion: "Thank you for talking with me today. Do you have any questions about what we did today?"

Turn off the recording. 


\section{Appendix E}

\section{Reverse Order Protocol}

Consent: Provide participant with informed consent. Say, "This basically tells you that your responses today are anonymous, that the session will be audio recorded, and that you can leave whenever you want to, without penalty. Please read and sign."

Introductory Phase: Introduce yourself. Something like: "Hi, my name is Keith. I'm a researcher with the psychology department. How are you?"

Develop rapport. Spend a couple of minutes talking with the participant. Draw some type of connection with yourself and the participant to make them feel comfortable speaking with you. For example:

"Did you find parking okay?"

"Do you live close to campus?"

"How is your week going?"

"That sounds [like a lot of work] [like a lot of fun] [like an interesting assignment]."

"Do you like that class? I took a similar class and really enjoyed it." (Any appropriate response to what the participant says)

$\underline{\text { Instructions }}$ Make sure to begin recording at this time.

"You watched a video a few minutes ago. That was a recording of someone committing a crime. What I'm going to do now is ask you some questions about what you saw. Okay, are you ready to begin?"

First free recall: "Please tell me everything you can remember about what you saw." Note: during this time, do not interrupt the participant! Take thorough notes.

Reverse order: "Okay, now I want you tell me everything that you saw, but in backward (reverse) order. [pause] So, start with the end of the crime you saw, and go backward to the beginning. Ok, just to make sure you understand, can you tell me what you're supposed to do?"

Make sure the participant understands the instruction; if not, repeat.

Last question: "Okay, is there anything else you can tell me about what you saw?" If the participant reports anything else, keep repeating the question. Repeat until participant responds no.

Conclusion: "Thank you for talking with me today. Do you have any questions about what we did today?"

Turn off the recording. 


\section{Appendix F}

\section{Instructions Condition Protocol}

Consent: Provide participant with informed consent. Say, "This basically tells you that your responses today are anonymous, that the session will be audio recorded, and that you can leave whenever you want to, without penalty. Please read and sign."

Introductory Phase: Introduce yourself. Something like: "Hi, my name is Keith. I'm a researcher with the psychology department. How are you?"

Develop rapport. Spend a couple of minutes talking with the participant. Draw some type of connection with yourself and the participant to make them feel comfortable speaking with you. For example:

"Did you find parking okay?"

"Do you live close to campus?"

"How is your week going?"

"That sounds [like a lot of work] [like a lot of fun] [like an interesting assignment]."

"Do you like that class? I took a similar class and really enjoyed it." (Any appropriate response to what the participant says)

$\underline{\text { Instructions }}$ Make sure to begin recording at this time.

"You watched a video a few minutes ago. That was a recording of someone committing a crime. What I'm going to do now is ask you some questions about what you saw.

Transfer of control: "This is not like a TV interview. I'm not going to ask a lot of questions. Instead, I want you to do most of the talking. You saw the crime, so you know what happened. I don't know what happened, so I need you to tell me. I'm just going to take notes while you talk.

Report everything instruction: "Every detail that you can remember is important. I want you to tell me everything you can possibly remember, even if it seems small. Don't guess, but make sure to tell me everything you can remember. We have a lot of time, so take as much time as you need.-Okay, are you ready to begin?"

First free recall: "Please tell me everything you can remember about what you saw." Note: during this time, do not interrupt the participant! Take thorough notes.

Second free recall: "Okay, now I want you to really think about everything you saw. [pause] Please tell me, again, everything that you remember about what you saw."

Last question: "Okay, is there anything else you can tell me about what you saw?" If the participant reports anything else, keep repeating the question. 
Repeat until participant responds no.

Conclusion: "Thank you for talking with me today. Do you have any questions about what we did today?"

Turn off the recording. 


\section{Appendix G}

Qualtrics Questionnaire

1. How hard was the interview overall?

$\begin{array}{lccccccccc}1 & 2 & 3 & 4 & 5 & 6 & 7 & 8 & 9 & 10 \\ \text { Very easy } & & & & & & & \text { Very hard }\end{array}$

2. How hard was it to [recall the second time $\mid$ reverse the order of your story $\mid$ report everything you could remember $]^{4}$ ?

$\begin{array}{lclllllllr}1 & 2 & 3 & 4 & 5 & 6 & 7 & 8 & 9 & 10 \\ \text { Very easy } & & & & & & & \text { Very hard }\end{array}$

3. What was the hardest part of the interview?

Speaking in English

Understanding the instructions

Remembering the event

[Reversing the order | Reporting everything | Reporting your story a second time] ${ }^{4}$

$\square$ Other:

4. How comfortable were you reporting what you saw in English?

$\begin{array}{cccccccccc}1 & 2 & 3 & 4 & 5 & 6 & 7 & 8 & 9 & 10 \\ \text { Very uncomfortable } & & & & & & \text { Very comfortable }\end{array}$

5. How correct do you think you were?

$\begin{array}{cccccccccc}1 & 2 & 3 & 4 & 5 & 6 & 7 & 8 & 9 & 10 \\ \text { Very incorrect } & & & & & & & & \text { Very correct }\end{array}$

\footnotetext{
${ }^{4}$ Question included piped text to reflect the participant's experimental condition.
} 


\section{IF participant was NNES:}

6. When you were being interviewed, did you mostly think in English or in your native language?

$\square$ English

$\square$ Native language

$\square$ Both

7. Please explain:

$<$ Provide space for participant to respond $>$ 


\section{Language \& Interviewing: Unitizing}

The purpose of coding transcripts is to find out (a) how much information the participant provided and (b) the type of information provided by participants. This task will help us answer our main research questions, so it is very important that you try your best and ask questions when you have them. Please do not guess or wing it!

The first step is to break down the transcripts into units of information. A unit of information is the smallest detail that can be checked for accuracy. Generally, each of the following will be defined as a unit:

\begin{tabular}{|l|l|l|}
\hline Noun & person, place, thing & the man, the office \\
\hline Adjective & description of a noun & color, size, etc. \\
\hline Active verb & $\begin{array}{l}\text { actions (not "was" or "had" } \\
\text { etc.) }\end{array}$ & walked, ran, grabbed, stole \\
\hline Adverb & description of an action & quickly, slowly, suspiciously \\
\hline Preposition & $\begin{array}{l}\text { clearly defines a relationship } \\
\text { between two things }\end{array}$ & $\begin{array}{l}\text { walked toward the building, laptop } \\
\text { was inside the bag }\end{array}$ \\
\hline
\end{tabular}

Special cases:

- Generally, IN and $\underline{\mathrm{ON}}$ do not need to be unitized. Ex: "in the hallway" or "on the door" each count as 1 unit.

- Adjectives separated by OR generally count as 1 unit. Ex: "in their 30s or 40s"

- Modifiers should stay in the same unit as the object/verb they modify.

O NOT: "they were not old" or "do not leave / the door / open"

○ Uncertainty: "they had a bag I think"

- Descriptions of the writing on the sign should be counted as 1 unit. Ex:

o leave the door closed when you leave the room

$\circ$ you cannot leave the doors open

$\circ$ etc. - Count all this as 1 unit.

- Descriptions of the participants' thoughts should be counted as 1 unit. Ex:

$\circ$ I'm not really sure what they were doing

(At this stage, you do not need to worry about whether the detail is correct. Just identify details that can be scored for accuracy.) 


\section{Example of a unitized transcript}

As you unitize, add automatic numbering to the transcript and add a line break after each unit. This should give us a (long but useful) transcript that looks something like this:

1. Okay ehm. There were two

2. persons

3. a man

4. and a woman

5. in an office

6. in in the psychology

7. department

8. of a university

9. or another place

10. i am not sure that it was a university

11. but it was in the psychology

12. department

13. there were in this in a room

14. and in a room

15. with a paper

16. on the door

17. that said that eh you can leave the doors closed when you when you leave the the room.

18. There were maybe taking

19. some

20. books

21. or something

22. that were there

23. or I'm not really sure what what were they doing

24 . but they suddenly

25 . they they just left

26. the office

27. they left

28. the door

29. open

30. and they were looking

31. back

32. to see

33. if some- someone

34. was looking

35. at them

36. and they just left. 


\section{Appendix I}

\section{Language \& Interviewing: Coding}

The purpose of coding transcripts is to find out (a) how much information the participant provided and (b) the type of information provided by participants. This task will help us answer our main research questions, so it is very important that you try your best and ask questions when you have them. Please do not guess or wing it!

The transcripts should unitized---i.e., they are basically long, numbered lists of details, like this:

1. Okay ehm. There were two

2. persons

3. a man

4. and a woman

5. in an office

6. in in the psychology

7. department

8. of a university

9. or another place

10. i am not sure that it was a university

First: Check if the information is NEW or REPEATED.

- $\quad$ REPEATED information ONLY refers to participants repeating previous details VERBATIM, except for The Sign

- Otherwise, the information is NEW

If the information is REPEATED: Mark it as repeated and move on with your life. Repeated information is not scored for accuracy or anything else.

If the information is NEW: Code it on the following dimensions.

\begin{tabular}{|l|l|l|}
\hline A & Accurate & the detail is true; verified via the master list and/or video \\
\hline I & Inaccurate & $\begin{array}{l}\text { the detail is incorrect; contradicts detail(s) from the master } \\
\text { list and/or video }\end{array}$ \\
\hline $\mathbf{S}$ & Subjective & the detail is an opinion; e.g., the perpetrator is "hot" \\
\hline $\mathbf{N}$ & Not scorable & an "other" category of things related to the video but \\
cannot be scored; e.g. "I can't remember" kind of stuff
\end{tabular}

These codes should be entered into the Google Sheets spreadsheet. Please follow the instructions on the coding sheet. 
Appendix $\mathbf{J}$

Correct Details

\begin{tabular}{|c|c|}
\hline Detail & Category \\
\hline Both look like students/seniors & Person \\
\hline Man Red/burgundy polo/shirt & Person \\
\hline Man heavy set/chunky/large stature/ possibly $190 \mathrm{LBS}$ & Person \\
\hline Man hispanic/white & Person \\
\hline Man Tall / 6'1"/6' & Person \\
\hline Man oval face & Person \\
\hline Woman heavy set/chunky/ about 155 LBS & Person \\
\hline Woman round face & Person \\
\hline Woman hispanic & Person \\
\hline Woman wearing gold ring on left ring finger & Person \\
\hline Woman Green wristband on right wrist & Person \\
\hline Woman hair length- mid back/shoulder length & Person \\
\hline Woman light pale skin & Person \\
\hline Woman short/5'5" & Person \\
\hline Man Plaid (carpenter) shorts & Person \\
\hline Man Long/shoulder length/split down the middle, dark (brown/black) hair & Person \\
\hline Man Beard/goatee/facial hair of medium length/Brown & Person \\
\hline Guy in flip flops & Person \\
\hline Woman Long red (dyed) hair, just past shoulders & Person \\
\hline Girl wavy hair/curly & Person \\
\hline Girl brown eyebrows & Person \\
\hline $\begin{array}{l}\text { Woman Floral shirt / blouse, short sleeves, open back/ cut-off in back, } \\
\text { purple flowers }\end{array}$ & Person \\
\hline Dark (black, navy, blue) jeans or leggings & Person \\
\hline
\end{tabular}




\begin{tabular}{|l|l|}
\hline COGLAB, CLAW, or 268 & Setting \\
\hline Yellow door & Setting \\
\hline Red Rug propped on wall & Setting \\
\hline Couch, chairs (2) & Setting \\
\hline Chairs (2) & Setting \\
\hline Open area with plants & Setting \\
\hline People sitting/laying in foyer (3) & Setting \\
\hline Psychology department & Setting \\
\hline Filing cabinets in hallway & Setting \\
\hline Two people in an office & Setting \\
\hline Room \#292 & Setting \\
\hline Office has windows/glass walls/sliding glass door & Setting \\
\hline Sign says "Attention: Please make sure that both sliding doors are locked \\
when you leave this room. Thank you, Psych Dept." & Setting \\
\hline Table and 2 chairs in office & Setting \\
\hline Another hallway w/ filing cabinets & Setting \\
\hline See thieves walk out through Psi Chi office & Setting \\
\hline They walk past a picnic table & Setting \\
\hline Walking around Building & Setting \\
\hline Cork Board on wall by where they leave & Setting \\
\hline Man takes 1 book & Theft \\
\hline Woman looking at book & Theft \\
\hline Man looking through bag & Theft \\
\hline Man takes 1 laptop & Theft \\
\hline Woman takes bag (messenger bag/ crossbody/ backpack) & Theft \\
\hline Bag is green/hunter green & Theft \\
\hline Woman takes 2 books & Theft \\
\hline
\end{tabular}




\begin{tabular}{|l|l|}
\hline Man glances backward/over shoulder & Theft \\
\hline Woman glances backward & Theft \\
\hline They look rushed & Theft \\
\hline They both glance backward & Theft \\
\hline He/she/they exit to outside & Theft \\
\hline Man looking at book & Theft \\
\hline women looking through bag & Theft \\
\hline
\end{tabular}


VITA

\section{BRYAN KEITH WYLIE}

2014

B.S., Psychology

University of Central Florida

Orlando, Florida

2017

M.S., Psychology

Florida International University

Miami, Florida

2017

Certificate in University Teaching and Learning

Center for Advancement of Teaching

Florida International University

Miami, Florida

\section{PUBLICATIONS AND PRESENTATIONS}

Wylie, K. \& Evans, J. (2019, June). Assessing cognitive interview mnemonics and their effectiveness with non-native English speakers. Poster presented at the Society for Applied Research in Memory and Cognition Conference, Cape Cod, MA.

McCarthy, R., Skowronski, J., Hoogesteyn, K., Verschuere, B., Meijer, E. H., Jim, A., ..., Charman, S. D., ..., Evans, J. R., ..., Wylie, K., \& Yildiz, E. (2018). Registered replication report on Srull and Wyer (1979). Advances in Methods and Practices in Psychological Science, 1, 299-317. doi:10.1177/2515245918781032

Verschuere, B., Meijer, E. H., Jim, A., McCarthy, R., Hoogesteyn, K., Skowronski, J., Orthey, R., ..., Charman, S. D., ..., Evans, J. R., ..., Wylie, K., \& Yildiz, E. (2018). Registered replication report on Mazar, Amir, and Ariely (2008). Advances in Methods and Practices in Psychological Science, 1, 321-336. doi:10.1177/2515245918777487

Wylie, K. \& Charman, S. (2018, March). Improving witnesses' lineup identification decisions with a 'not sure' instruction: The moderating role of memory strength. Paper presented at the American Psychology-Law Society Conference, Memphis, TN.

Wylie, K. \& Evans, J. (2017, March). Cognitive load and the misinformation effect. Paper presented at the American Psychology-Law Society Conference, Seattle, WA.

Charman, S., Cahill, B., Wylie, K., Kekessie, S., Waggoner, B., \& Perez, C. (2016, March). An explicit 'not sure' option reduces false identifications and eliminates the harmful effects of the appearance change instruction. Paper presented at the American Psychology-Law Society Conference, Atlanta, GA. 
Charman, S., Cahill, B., Wylie, K., Waggoner, B., \& Perez, C. (2016, March). An explicit 'not sure' option reduces mistaken lineup identifications and eliminates the harmful effects of the appearance change instruction. Paper presented at the International Forensic Research Institute's Forensic Science Symposium, Miami, FL.

Wylie, K. \& Evans, J. (2016, March). Exploring the impact of cognitive load on interrogative suggestibility. Paper presented at the American Psychology-Law Society Conference, Atlanta, GA.

Molinaro, P., Charman, S., \& Wylie, K. (2015, March). Is the relationship between prelineup confidence and accuracy as tenuous as it appears? Paper presented at the American Psychology-Law Society Conference, San Diego, CA.

Wylie, K., Cooper, T., Reeves, D., Baker, A., \& Mouloua, M. (2014, March). An exploration of the effect of media bias on individual jurors' predetermination of guilt. Poster presented at the Southeastern Psychological Association, Nashville, TN. 